\title{
Deep Geothermal Heating Potential for the Communities of the Western Canadian Sedimentary Basin
}

\author{
Jacek Majorowicz ${ }^{1,2, *}$ and Stephen E. Grasby ${ }^{3}$ \\ 1 Northern Geothermal Cons, Edmonton, AB T6R2J8, Canada \\ 2 Department of Physics, University of Alberta, 11322-89 Ave., Edmonton, AB T6G 2G7, Canada \\ 3 Geological Survey of Canada, 330333 St NW, Calgary, AB T2L 2A7, Canada; steve.grasby@canada.ca \\ * Correspondence: majorowi@ualberta.ca or majorowicz@shaw.ca
}

check for

updates

Citation: Majorowicz, J.; Grasby, S.E. Deep Geothermal Heating Potential for the Communities of the Western Canadian Sedimentary Basin.

Energies 2021, 14, 706. https:// doi.org/10.3390/en14030706

Academic Editor: Stefano Mazzoli Received: 27 December 2020

Accepted: 27 January 2021

Published: 30 January 2021

Publisher's Note: MDPI stays neutral with regard to jurisdictional claims in published maps and institutional affiliations.

Copyright: (c) 2021 by the authors. Licensee MDPI, Basel, Switzerland. This article is an open access article distributed under the terms and conditions of the Creative Commons Attribution (CC BY) license (https:/ / creativecommons.org/licenses/by/ $4.0 /)$.

\begin{abstract}
We summarize the feasibility of using geothermal energy from the Western Canada Sedimentary Basin (WCSB) to support communities with populations $>3000$ people, including those in northeastern British Columbia, southwestern part of Northwest Territories (NWT), southern Saskatchewan, and southeastern Manitoba, along with previously studied communities in Alberta. The geothermal energy potential of the WCSB is largely determined by the basin's geometry; the sediments start at $0 \mathrm{~m}$ thickness adjacent to the Canadian shield in the east and thicken to $>6 \mathrm{~km}$ to the west, and over $3 \mathrm{~km}$ in the Williston sub-basin to the south. Direct heat use is most promising in the western and southern parts of the WCSB where sediment thickness exceeds $2-3 \mathrm{~km}$. Geothermal potential is also dependent on the local geothermal gradient. Aquifers suitable for heating systems occur in western-northwestern Alberta, northeastern British Columbia, and southwestern Saskatchewan. Electrical power production is limited to the deepest parts of the WCSB, where aquifers $>120{ }^{\circ} \mathrm{C}$ and fluid production rates $>80 \mathrm{~kg} / \mathrm{s}$ occur (southwestern Northwest Territories, northwestern Alberta, northeastern British Columbia, and southeastern Saskatchewan. For the western regions with the thickest sediments, the foreland basin east of the Rocky Mountains, estimates indicate that geothermal power up to $2 \mathrm{MW}_{\mathrm{el}}$. (electrical), and up to 10 times higher for heating in $\mathrm{MW}_{\text {th. }}($ thermal), are possible.
\end{abstract}

Keywords: heat flow; deep geothermal heat; foreland basin; WCSB; energy transfer

\section{Introduction}

Direct heating with geothermal energy could provide an important energy resource in cold climate regions, such as the Canadian prairie provinces where heating accounts for $80 \%$ of the total energy demand. Sedimentary basins, such as the Western Canadian Sedimentary basin (WCSB), hold significant heat that could be used to support communities overlying the basin. Direct use geothermal energy is commonly used for district heating systems [1-4]. Typically, district heating systems require temperatures $>60^{\circ} \mathrm{C}$ and fluid production rates $>30 \mathrm{~kg} / \mathrm{s}$, using two or more geothermal wells with at least one production well and one injection well [5].

District heating systems could significantly reduce $\mathrm{CO}_{2}$ emissions by replacing gas and oil combustion with renewable energy resources [6-11]. The WCSB contains large geothermal energy reserves, with temperatures reaching over $160^{\circ} \mathrm{C}$. About half of the basin area is $>2 \mathrm{~km}$ deep, with measured temperatures $>60{ }^{\circ} \mathrm{C}$ [11-16].

Previous studies of the WCSB examined both direct heating energy, in GJ per year, and potential electrical power generation (MW electrical), but for only the province of Alberta [17]. We expand on this previous work by examining geothermal potential for all communities with populations $>3000$ people that overly the WCSB, adding geothermal energy calculations for northeastern British Columbia, Saskatchewan, southern Manitoba, and southwestern NWT (see location of the study area in Figure 1 and location of the municipalities in Figure 2). Maps specific to these new assessment areas are presented 
in Appendices A and B, while combined maps for the entire WCSB are presented here. Calculated geothermal potential for specific communities are in tables in Appendix C. These tables include geothermal energy available assuming an average energy use of $130 \mathrm{GJ} /$ year, enthalpy, calculated formation temperature, and the drill depth required for calculated temperatures.

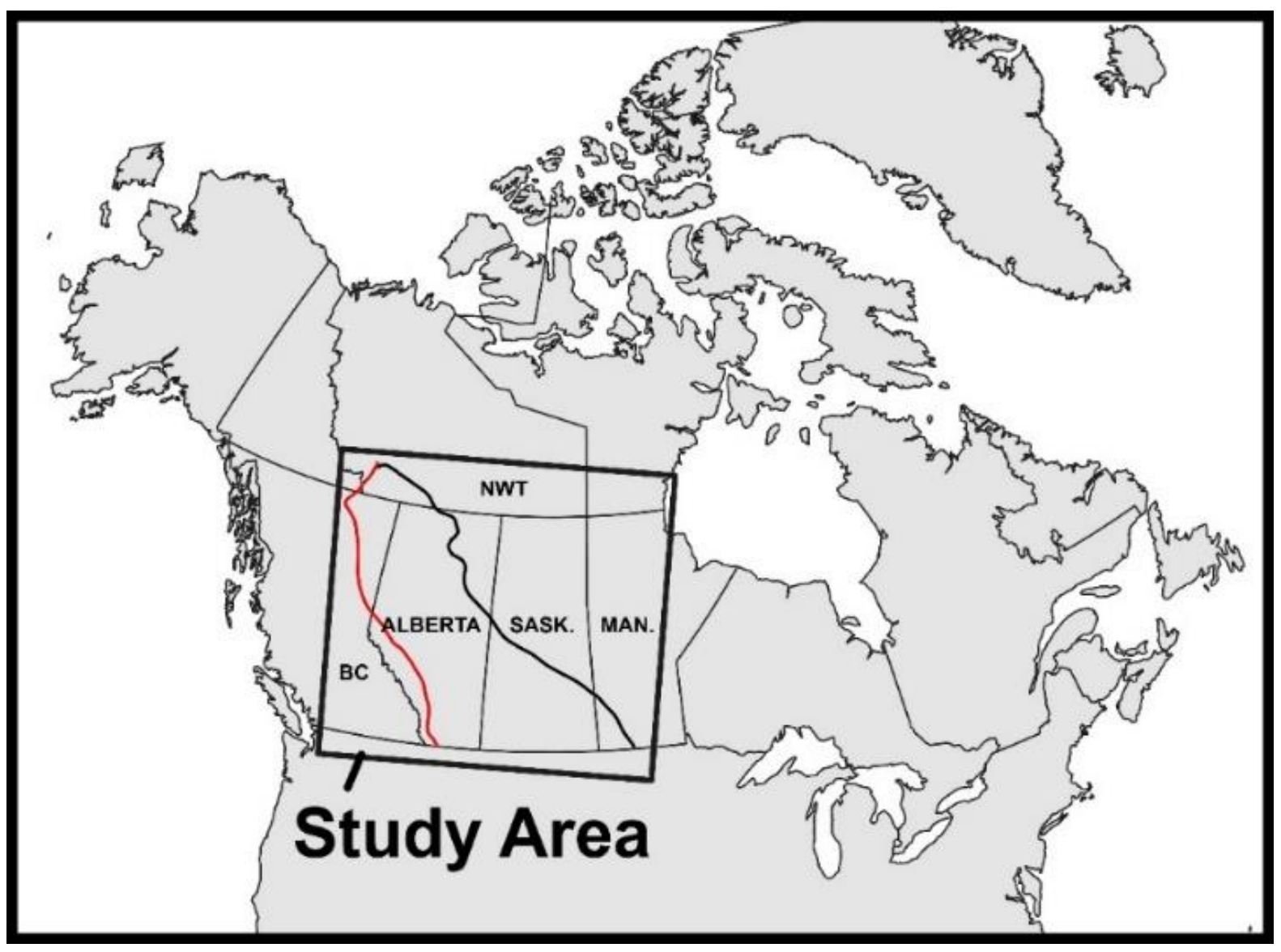

Figure 1. Map of Canada showing the study area (rectangle). Provinces and territories that are considered in the study are indicated along with the outline of the Western Canada Sedimentary Basin (WCSB). BC = British Columbia, NWT = Northwest Territories, Sask. = Saskatchewan, Man. = Manitoba. The red line depicts the western margin of the WCSB which is defined by the deformation front of the Canadian Rocky Mountains. The black line shows the eastern edge of our study defined by $1 \mathrm{~km}$ sedimentary thickness. 


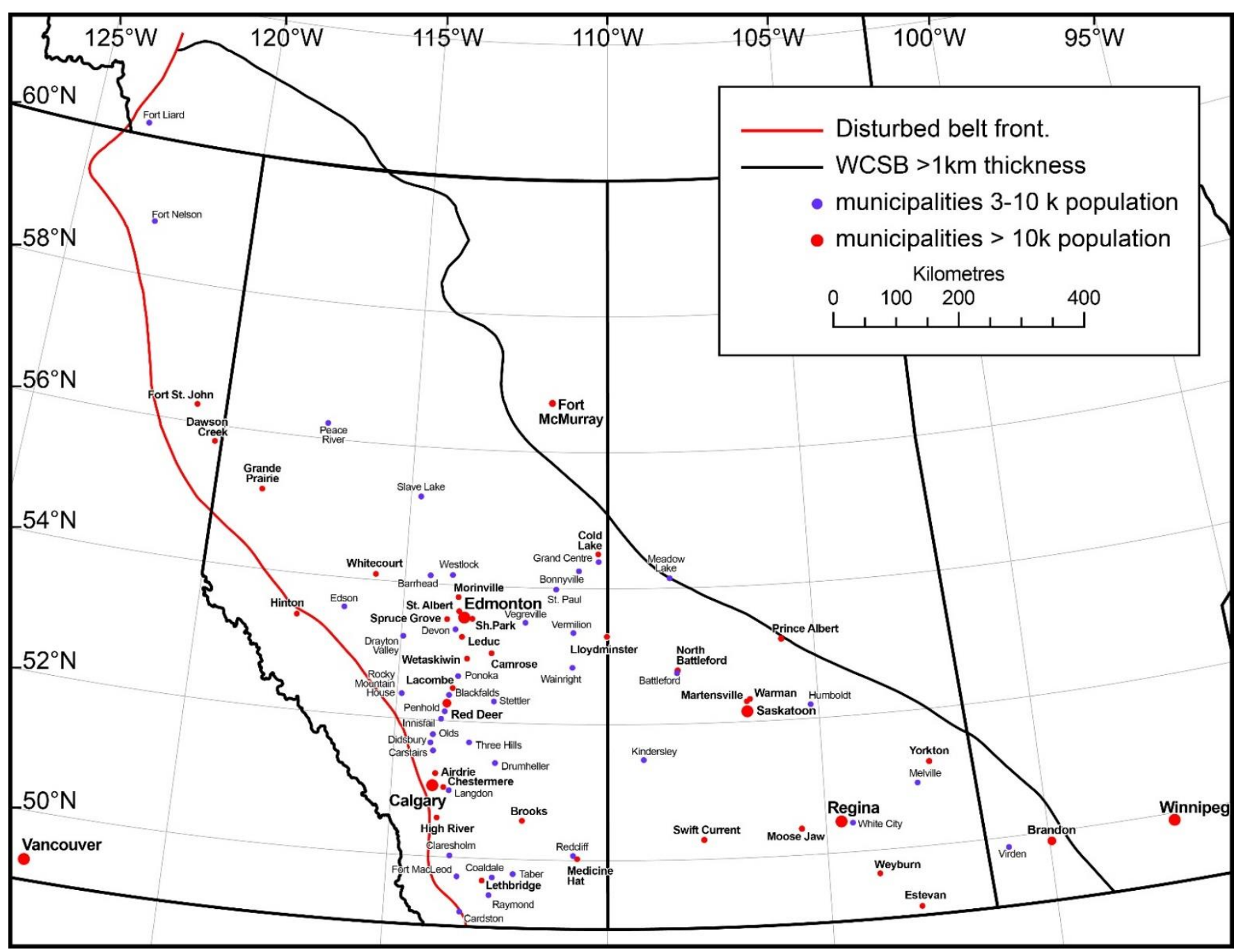

Figure 2. Location map of the Western Canadian Sedimentary Basin (WCSB) with provincial boundaries and locations of municipalities with populations $>3 \mathrm{k}$. The red line depicts the western margin of the WCSB defined by the deformation front of the Canadian Rocky Mountains.

\section{Background}

Geothermal production of electrical power, through the Organic Rankin (ORC) or Kalina cycle (KC), needs to be in the vicinity of an existing power grid to be economical. Such infrastructure is available in some remote areas as already shown by wind-based power production in Alberta. However, the low 10\% efficiency of ORC and KC power plants is a limiting factor, making only high enthalpy regions of interest for electrical potential, such as the deepest parts of the WCSB in Alberta and southern Saskatchewan [11]. However, "The Alberta Climate Leadership Plan" goal of replacing 5000 megawatts of coal-generated electricity with power coming from renewable sources by the year 2030 is daunting. Electrical power from geothermal sources would require thousands of geothermal doublet well installations, while two well systems with 1-2 MW potential is feasible in only limited areas (see Tables in Appendix C). The cost of geothermal wells to produce sufficient electricity would be upwards of $\$ 50$ billion dollars for 1000 systems [7]. District heating $(\mathrm{DH})$ may therefore be the most feasible use of geothermal resources in cold climate regions such as the Canadian prairie provinces. This also comes with challenges though.

Transmitting hot fluids over large distances comes with significant energy loss, which means that to be useful, DH projects must be as close to a community as possible [18,19]. Modelling by Kapil et al. [19] indicates that there is an $\sim 1 \%$ heat loss for every $\mathrm{km}$ of 
insulated pipe distance. However, the Kapil et al. [19] model did not consider the cost for pump operation to determine the economically feasible distance of heat transmission. Later work shows that distance needs to be even smaller for DH systems. Economic constraints mean that high enthalpy, high temperature $\left(120-250{ }^{\circ} \mathrm{C}\right)$ [20], steam can be transported $3-5 \mathrm{~km}$, water with temperatures $90-175^{\circ} \mathrm{C}$ some $30 \mathrm{~km}$, and waters with lower grade heat [21], $15 \mathrm{~km} \mathrm{[18].}$

\section{Structural Setting of the WCSB}

The WCSB underly 1,400,000 km² of Western Canada, (southwestern Manitoba, southern Saskatchewan, Alberta, northeastern British Columbia (BC) and the southwest corner of the Northwest Territories (NWT)). A massive wedge of sedimentary rock extends from the Rocky Mountains (Canadian Cordillera) in the west, to the Canadian Shield in the east. This wedge is about $6 \mathrm{~km}$ thick at the deepest part of the basin bordering the Cordillera but thins to zero $\mathrm{m}$ at its eastern margins in Manitoba, northeastern Saskatchewan, and southwestern NWT (see Figure 3 below). A geological cross-section perpendicular to the basin's strike shows the general configuration of the Pre-Cambrian basement and overlying sedimentary formations. We show in Figure 3 that a $2 \mathrm{~km}$ drilling depth will reach $60-70{ }^{\circ} \mathrm{C}$ fluids according to [22], while a $3 \mathrm{~km}$ depth will reach some $90-100{ }^{\circ} \mathrm{C}$.

A generalized stratigraphic column of the WCSB is also shown in Figure 3. Table 1 lists the Geological Period from Cretaceous down to Cambrian and the formations that are known to have significant permeability [15]. The tops of these formations and groups, their thickness maps and cross- sections, are readily available from the Alberta Geological Survey (AGS) online: <https://ags.aer.ca/reports/atlas-western-canada-sedimentary-basin>. The geological information is not repeated here as we focus on the thermal conditions of most the most prospective sedimentary groups.

Table 1. Potential geothermal target formations in the WCSB (modified from [15]).

\begin{tabular}{|c|c|c|c|}
\hline Period & Group & Formation & Lithology \\
\hline Cretaceous & Mannville & & sandstone \\
\hline Cretaceous & Mannville & Cadomin & sandst./congl. \\
\hline Mississippian & Rundle & & carbonates \\
\hline Mississippian & - & Charles & carbonates \\
\hline Mississippian & - & Banff & limestone \\
\hline Devonian & Wabamun & Wabamun & dolomite \\
\hline Devonian & Winterburn & Nisku & carbonates \\
\hline Devonian & Woodbend & Grosmont & dolomite \\
\hline Devonian & Woodbend & Leduc & dolomite \\
\hline Devonian & Woodbend & Cooking Lake & carbonates \\
\hline Devonian & Beaverhill & Slave Point & carbonates \\
\hline Devonian & Beaverhill & Swan Hills & carbonates \\
\hline Devonian & Elk Point & Pine Point & dolostone \\
\hline Devonian & - & Granite Wash & sandstone \\
\hline Cambrian & Lynx & Deadwood Fm. & sandstone \\
\hline Cambrian & - & Basal Sandstone & sandstone \\
\hline
\end{tabular}




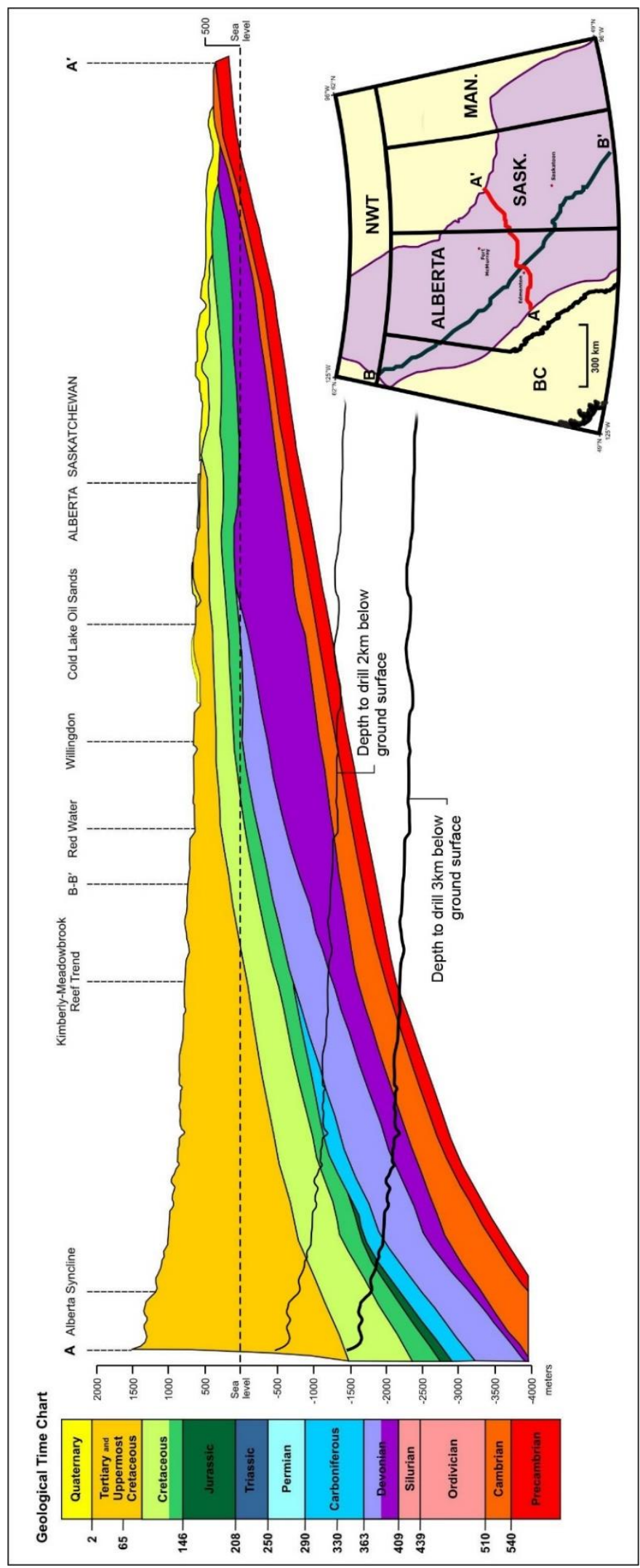

Figure 3. Geological cross-section through the Western Canadian Sedimentary Basin. The main geological formations are shown. Depth to drill to $2 \mathrm{~km} 60-70{ }^{\circ} \mathrm{C}$ below the surface and $3 \mathrm{~km}$ $90-100{ }^{\circ} \mathrm{C}$ is indicated. 


\section{Geothermal Gradient and Maximum Temperatures-WCSB}

The heat flow $Q$ map of the study area $[10,23]$ is plotted in Figure 4. Locations of municipalities studied here, those with populations $>3000$ people, are shown on a map of average geothermal gradient (Grad T(z), where T-temperature, $\mathrm{z}$ - depth) of the WCSB in Figure 5. The map of Grad T(z) is based on industrial temperature logs, corrected bottom hole temperature data, drill stem test temperature records, and shut-in wells temperature data from tens of thousands of boreholes drilled for oil and gas $[9,16,23,24]$.

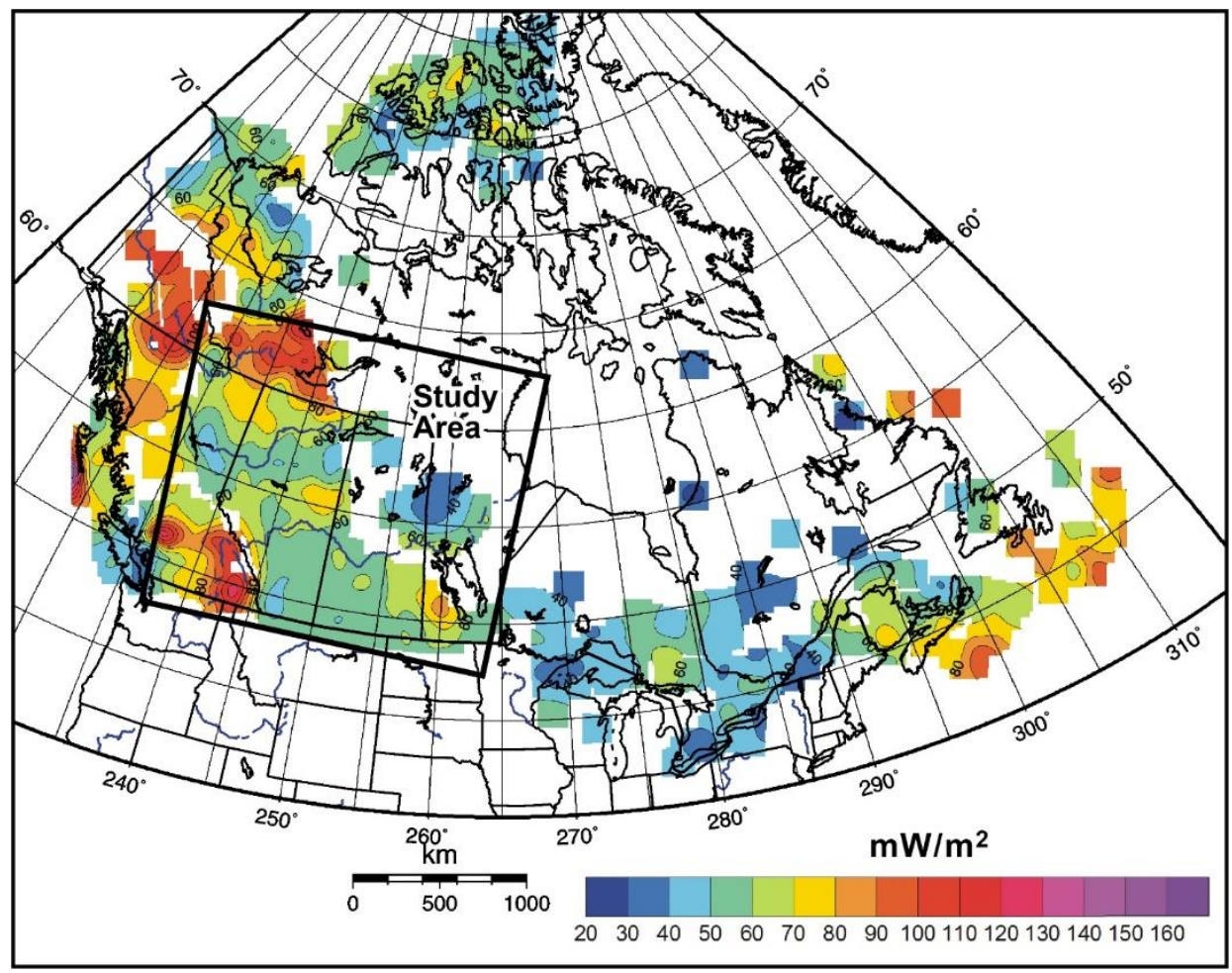

Figure 4. Study area against the map of heat flow Q of Canada (modified from [10]).

Most of the municipalities are in the southern part of the WCSB, where thermal gradients are low $\left(20{ }^{\circ} \mathrm{C} / \mathrm{km}\right)$ to moderate $\left(30-45^{\circ} \mathrm{C} / \mathrm{km}\right)$ in southern Alberta. The central western part of basin is $>2.5 \mathrm{~km}$ deep and has elevated $\operatorname{GradT}(\mathrm{z})$ of $>35^{\circ} \mathrm{C} / \mathrm{km}$. In southeastern Saskatchewan, the basin has an elevated Grad $\mathrm{T}(\mathrm{z})$ of $40{ }^{\circ} \mathrm{C} / \mathrm{km}$. The northwestern part of the basin has just a few communities, like Fort Nelson and Fort Liard, which occur in areas of elevated $\operatorname{GradT}(\mathrm{z})\left(40-50^{\circ} \mathrm{C} / \mathrm{km}\right)$. Geothermal gradients of $35-50{ }^{\circ} \mathrm{C} / \mathrm{km}$ for large parts of the WCSB are high compared to other sedimentary basins worldwide [25].

The Precambrian basement which underlies the WCSB has radiogenic heat generation two times higher than in outcrops of the correlative Canadian Shield [23-26]. Radiogenic heat production $(A)\left[\mu \mathrm{W} / \mathrm{m}^{3}\right]$ in the Precambrian basement underlying the WCSB shows large variability but averages $2.1-2.4 \mu \mathrm{W} / \mathrm{m}^{3}$. This explains the higher heat flow of the WCSB as compared to the Canadian Shield to the east.

The temperature distribution in sediments of the WCSB is determined from ground surface temperature records [27], the WCSB heat flow Q map [16,23], and the WCSB thermal conductivity $\mathrm{k}$ map [23]. Some $40-50 \%$ of $\mathrm{Q}$ is from radiogenic heat in the crust and $50-60 \%$ comes from deeper sources in continental settings [28-30]. The review of Epelbaum et al. [31] suggests mantle heat flow is in the order of $15-84 \%$ of total. Heat flow determinations in Canada are based on single heat flow determinations in wells and group of wells from industrial temperature records. Some areas thus lack data (Figure 4) due to its remoteness and/or lack of drilling [10]. While the North America heat flow 
map extrapolates over large areas of Canada with no data [32], these regions are shown in white here.

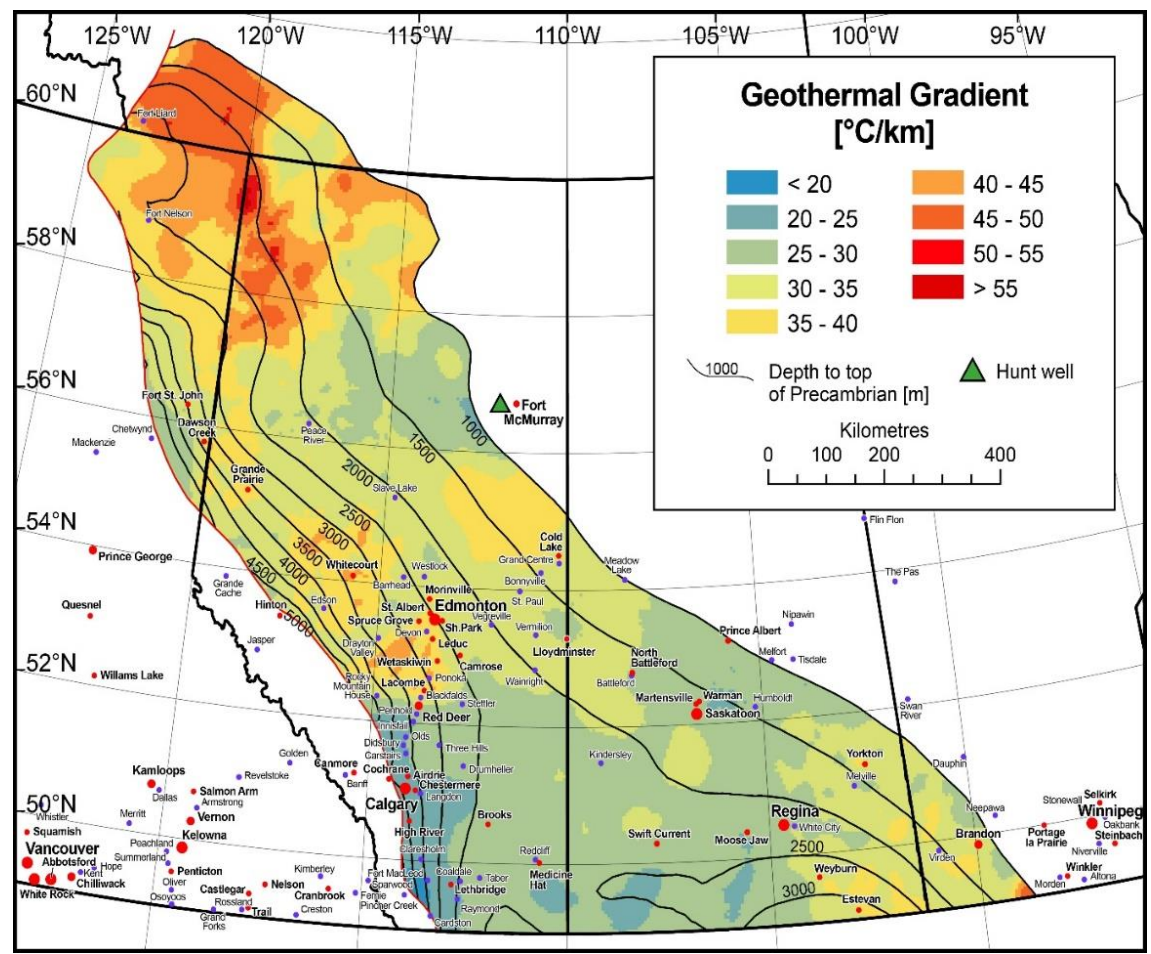

Figure 5. The communities' studies here shown against the map of average geothermal gradient for the WCSB with sediment thickness $>1000 \mathrm{~m}$. The red line is the western boundary of the WCSB marked by the Rocky Mountains disturbed belt modified from [16]. The Hunt well near Fort McMurray is the site of first heat flow determined below the WCSB, in an interval of the $0.5-2.4 \mathrm{~km}$ in Precambrian granites [33].

The only high precision deep $(>1 \mathrm{~km})$ heat flow results in the WCSB are from the deep $(2.3 \mathrm{~km})$ Hunt well near Fort McMurray (Figure 5), that shows surface heat flow of $\mathrm{Q}=57 \mathrm{mWm}^{-2}$ [33], significantly higher than $\mathrm{Q}$ in the Canadian Shield to the east $\left(44 \pm 7 \mathrm{mWm}^{-2}\right.$, [25]). This result is interpreted as being related to the high average A of $2.9 \mu \mathrm{Wm}^{-3}$ in the upper granitic crust of the well [33]. The rest of heat flow data for the WCSB come from thousands of single depth bottom hole and drill stem test temperatures and effective thermal conductivity estimates based on net rock data and measured rock conductivities of typical lithologies [10,12,16,23].

Since geothermal gradient is defined by Equation (1):

$$
\operatorname{GradT}(\mathrm{z})=\mathrm{Q} / \mathrm{k}
$$

Thermal conductivity $\mathrm{k}$ will control Grad $\mathrm{T}(\mathrm{z})$ at constant heat flow $\mathrm{Q}$. Thermal conductivity of the sedimentary fill $\left(\mathrm{k}_{\mathrm{sed}}\right)$ of the WCSB was studied for sedimentary rocks and a map of $\mathrm{k}_{\text {sed }}$ pattern was constructed [23]. Thermal conductivity of sediments $\mathrm{k}_{\text {sed. }}$ of Cenozoic, Mesozoic, and upper to lower Paleozoic rocks varies in relationship with the overall composition from low $\mathrm{k}$ of shales $(1.2 \mathrm{~W} / \mathrm{m} \mathrm{K})$ to high $\mathrm{k}$ of carbonates $(3 \mathrm{~W} / \mathrm{m} \mathrm{K})$, quartzite sediments $(4-6 \mathrm{~W} / \mathrm{m} \mathrm{K})$, and salt $(7 \mathrm{~W} / \mathrm{m} \mathrm{K})$. The variability in the thickness of lithostratigraphic units, changes in sedimentary facies, and the presence or absence of sedimentary units, results in variability of net $\mathrm{k}$ with depth. Calculated $\mathrm{k}_{\text {sed. }}$ shows a trend of increasing eastward towards the shield. Some very low k zones, like in the northwestern Alberta-BC part of the basin $\left(\mathrm{k}_{\mathrm{sed}}=1.4-1.6 \mathrm{~W} / \mathrm{m} \mathrm{K}\right)$, can explain some of the highest GradT observed in the WCSB (Figure 5). Very high integrated $\mathrm{k}_{\text {sed. }}(2.6-2.8 \mathrm{~W} / \mathrm{m} \mathrm{K}$ ) in the eastern shallow parts of the WCSB are close to the $\mathrm{k}$ of underlying Precambrian basement, 
explaining the low temperatures at depth in those areas [22]. The areas with the lowest net $\mathrm{k}$ are prospective for high temperature geothermal systems, as the rate of temperature increase with depth are the highest for constant heat flow (Equation (1)). The highest heat flow-lowest $\mathrm{k}_{\text {sed. }}$ areas have the highest temperatures and thermal heat storage. In general, the westward increasing thickness of the WCSB, and decreasing net $\mathrm{k}_{\text {sed. }}$ increases available thermal energy.

Below the WCSB, the average $\mathrm{k}$ of basement rocks [26] is significantly higher. The highest average conductivity is that of igneous rocks $(3.4 \mathrm{~W} / \mathrm{m} \mathrm{K} \mathrm{N}=56)$ and then metamorphic rocks $(3.2 \mathrm{~W} / \mathrm{m} \mathrm{K}, \mathrm{N}=146)$. At the observed average heat flow of the Alberta basin $\left(70 \mathrm{~m} \mathrm{~W} / \mathrm{m}^{2}\right)$ this would cause changes in the geothermal gradient below the Pre-Cambrian surface of $28^{\circ} \mathrm{C} / \mathrm{km}$ to $19^{\circ} \mathrm{C} / \mathrm{km}$, respectively.

\section{Geothermal Energy Calculation}

We calculated geothermal energy for each of the municipalities in our study area. An outflow fluid temperature of $60^{\circ} \mathrm{C}$ was assumed for geothermal heating systems based on the upper limit of the Paris DH system [2,4]. For electrical production we assumed 50-60 ${ }^{\circ} \mathrm{C}$ for an ORC electrical power plant system according to Tester et al. [5]. The range of the feasible net geothermal heat and geothermal electrical power production was calculated using parameters from Table 2 and temperatures derived from maps in Figures 6 and $7 \mathrm{a}-\mathrm{d}$. In previous geothermal assessments the specific heat capacity $\left(\mathrm{C}_{\mathrm{W}}\right)$ of low salinity waters was used $\left(4200 \mathrm{~J} / \mathrm{kg} / \mathrm{K}\right.$ in [11]. Here we adjusted $\mathrm{C}_{\mathrm{W}}$ to lower values (3150 J/( $\mathrm{kg} \mathrm{K}) — 3993 \mathrm{~J} /(\mathrm{kg} \mathrm{K}))$, [34] that more accurately reflect the brines which occur in the most perspective geological formations we examined [6,35-42]. To calculate these $\mathrm{C}_{\mathrm{W}}$ values we used the MIT charts [34]. Feasible flow rates used were based on various published pumping test results for the WCSB, as well as in analogous basins in the USA and Germany, as summarized by Majorowicz and Grasby [11].

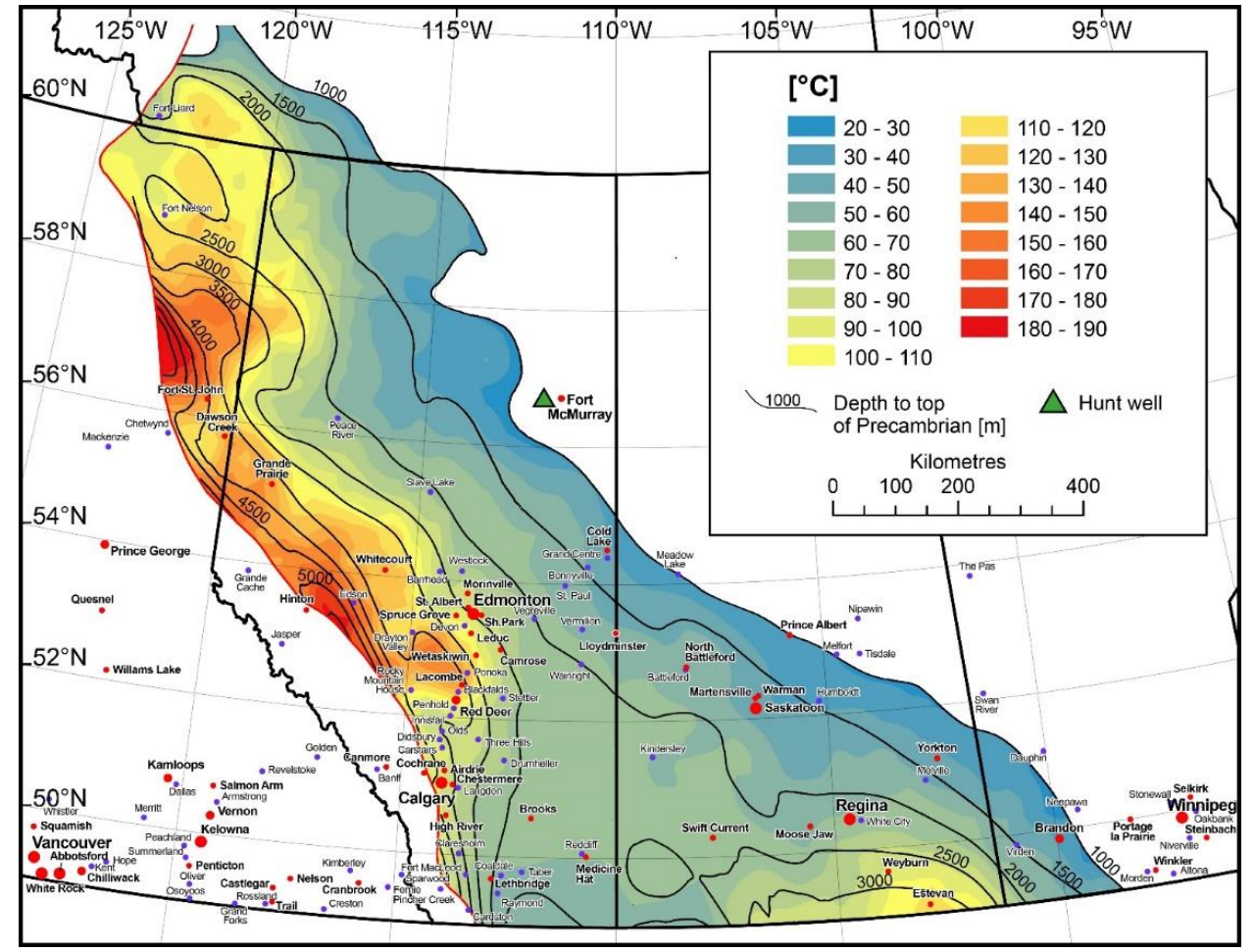

Figure 6. WCSB municipalities $>3 \mathrm{k}$ population plotted against the calculated temperature $\left[{ }^{\circ} \mathrm{C}\right]$ pattern at the base of the Phanerozoic $=$ top of Precambrian basement. Red line depicts western reach of the WCSB which is at the Rocky Mountains deformation front. 


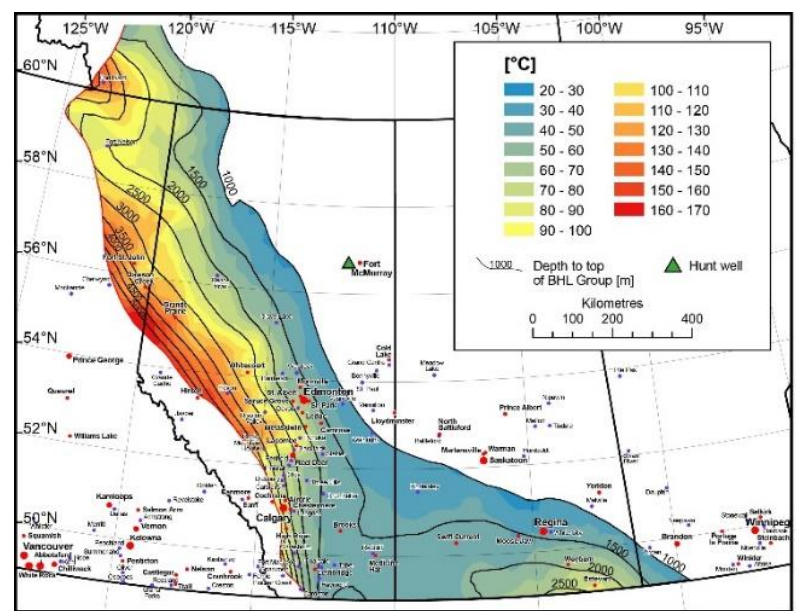

a)

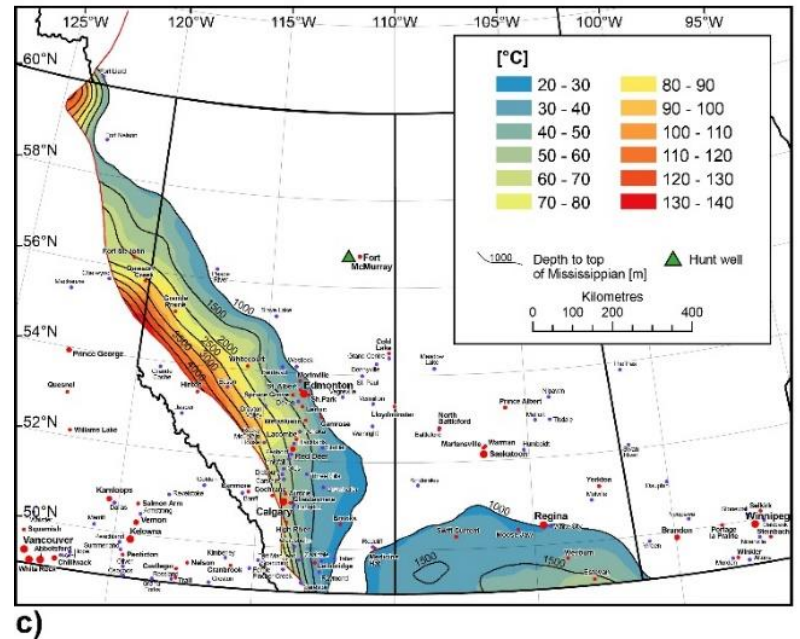



b)

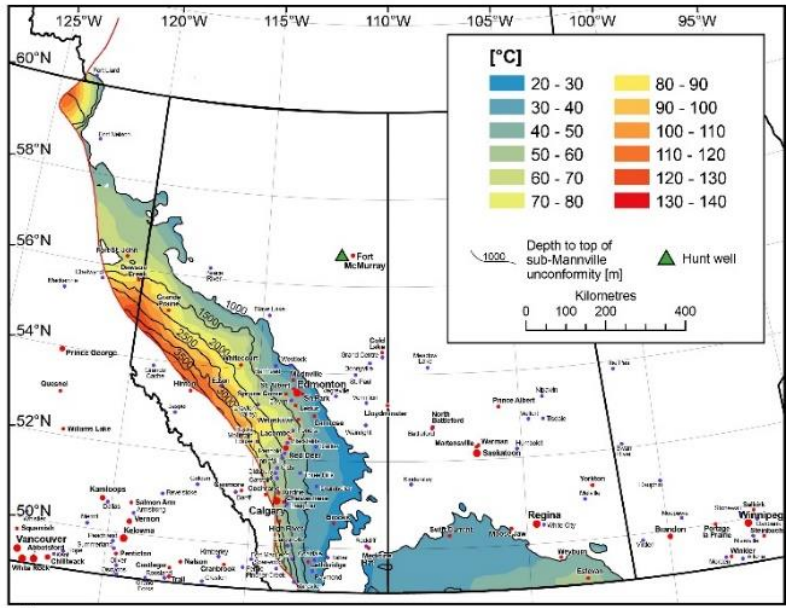

d)

Figure 7. WCSB municipalities with $>3 \mathrm{k}$ population plotted against the maps of temperature at various geological surfaces [15]; (a) The base of Upper Devonian Beaverhill Lake Group; (b) The top of the Upper Devonian Winterburn Group; (c) The top of Mississippian formations; (d) at the sub-Mannville unconformity. Note: The red line depicts western margin of the undeformed WCSB.

Table 2. Assumed parameters.

\begin{tabular}{ccc}
\hline Parameter & Range & Unit \\
\hline Production temperature of geothermal fluid & $70-160$ & ${ }^{\circ} \mathrm{C}$ \\
\hline Backflow temperature & $50-60$ & ${ }^{\circ} \mathrm{C}$ \\
\hline Specific heat capacity & $3150-3993$ & $\mathrm{~J} / \mathrm{kg}{ }^{\circ} \mathrm{C}$ \\
\hline Flow rate & $30-80$ & $\mathrm{~kg} / \mathrm{s}$ \\
\hline Conversion MWthermal to MWelectrical factor ${ }^{1}$ & $8-12$ & $\%$ \\
\hline
\end{tabular}

${ }^{1}$ common for the Organic Rankin cycle power plants [5].

\section{WCSB Prospects-Summary}

The map of communities vs. highest available temperatures at the base of the Phanerozoic of the WCSB, above the Precambrian basement, is shown in Figure 6. Similar maps are also shown for the Middle Cambrian and Upper Cambrian, Devonian Granite Wash Formation (Figure 6), base of Beaverhill Lake Group (Figure 7a), top of the Upper Devonian Winterburn Group (Figure 7b), top of Mississippian formations (Figure 7c), and at the sub-Mannville unconformity (Figure 7d). 
For most of the study area, the highest temperature zones are found in the deep foreland basin adjacent to the Rocky Mountains in western Alberta [15,39,40] and in the Williston sub-basin of southern Saskatchewan. The calculated geothermal energy is shown in Table A3 (Appendix C). Results in Table A2 (Appendix C) show that temperatures $>80^{\circ} \mathrm{C}$ can be found in the Upper Devonian Beaverhill Lake Group [15,41]. Other potential formations with geothermal feasibility include the Woodbend Group's Grossmond karstic dolomites, Cooking Lake dolomites, the Beaverhill Lake's Group Swan Hill Formation, Slave Lake Formation, and the Elk Point's Group Pine Point Formation [15]. The Winterburn Group, and Wabamun and Nisku formations geothermal prospects for heating and electrical power are shown in Table A5. The Wabamun group's partly porous dolomites [15], and Winterburn group's Nisku Formation sandstones and limestones [15] have sufficient temperatures (Figure 7) to form geothermal energy prospects west of Edson and to the northwest towards Grande Cache (Table A3). The Mississippian Rundle Group's dolostones and limestones, Charles Formation dolostones and limestones, and Banff Formation limestones [15] are all in the $>80{ }^{\circ} \mathrm{C}$ temperature zone in the deepest western part of the WCSB in Alberta (Figure 7c). The calculated energy, power and enthalpy gains for these units are shown in Table A4 (Appendix C). Low to mid-enthalpy potential for geothermal hot saline fluids also exists for the Cretaceous Mannville Group sediments and Cadomin Formation sandstone \& conglomerates, as found by Lam and Jones, [37], in the deepest parts of the WCSB in the Edson-Hinton area. The sub-Manville unconformity temperature distribution in Figure $7 \mathrm{~d}$ shows temperatures $>70{ }^{\circ} \mathrm{C}$ that are useable for geothermal heating for the towns of Drayton Valley, Edson, and Rocky Mountain House (Table A5 (Appendix C)).

\section{Discussion of Results}

A summary of results is presented in Tables A1-A5 in Appendix C. Results show that there are many municipalities that could potentially exploit deep geothermal heat reserves, and fewer cases for electrical production (Fort Liard NWT: Hinton Alberta, Estevan Saskatchewan). However, there are many regions of the WCSB with good electrical potential, but without nearby populated areas, which would increase transmission costs.

Analysis of geothermal feasibility for municipalities show that geothermal heat is available from several geological formations (Tables A4 and A5 and Figures 6 and 7c,d). In parts of the deep basin in western Alberta, the Granite Wash, Middle Cambrian basal sandstone, Winnipegoisis, and Deadwood formations [15,39-42] reach temperatures of $140-170{ }^{\circ} \mathrm{C}$ close to the municipalities of Hinton, Edson, Grand Prairie, Rocky Mountain House, and Whitecourt (Table A3). Temperatures $>90{ }^{\circ} \mathrm{C}$ (Figure 7a) are also found for the BeaverHill Lake Group (Table A2). Temperatures sufficient or direct heating prospects occur in the Devonian Winterburn/Wabamun groups (Figure 7b; Table A3). Temperatures $>70{ }^{\circ} \mathrm{C}$ are found in western Alberta in the Rundle Group's Charles Formation dolostones and limestones, as well as Banff Formation limestones) (Table A4).

In Table A5 we summarize energy calculations for the formations above the subManville unconformity. The potential for geothermal hot saline fluids exists for the Mannville and Cadomin sandstones and conglomerates. As we move from deep formations above the Precambrian surface up towards shallower formations, like the Mississippian and/or Mannville prospects for direct heating, many municipalities' drop off the list (Tables A4 and A5) due to too low local temperatures for $\mathrm{DH}$ systems $\left(<70{ }^{\circ} \mathrm{C}\right)$ or that the municipality lies outside of the formation sub-crop boundaries in the eastern and northeastern parts of the WCSB (see Tables A3 and A4).

Depending on the temperature of deep aquifers $\mathrm{T}\left({ }^{\circ} \mathrm{C}\right)$ and production rates $(\mathrm{kg} / \mathrm{s})$ (Tables A1-A5) there is a whole range of possibilities to use geothermal energy for direct heating. The calculated number of households feasible to be heated by a direct deep-aquifer sourced geothermal energy is in $100 \mathrm{~s}$ to $1000 \mathrm{~s}$ for Alberta, BC, and NWT, as well as deep parts of the Williston Basin sub-basin in southern Saskatchewan. 
The calculated enthalpy gains for all the communities overlying the WCSB are given in Appendix C Tables A1-A5 and they are ranked 1-5 as listed below [43]:

1. Very low enthalpy gain $<80 \mathrm{~kJ} / \mathrm{kg}$ - aquifer,

2. Low to Medium enthalpy (80-200) $\mathrm{kJ} / \mathrm{kg}$ - Geothermal heat prospects with uplift by heat pumps

3. Medium enthalpy prospects (200-320) kJ/kg - Prospects for direct deep aquifer source based geothermal heating.

4. High enthalpy $(320-520 \mathrm{~kJ} / \mathrm{kg})$ - Very good direct heat prospects, marginal EGS geothermal electrical power prospects.

5. Very high enthalpy ( $>520 \mathrm{~kJ} / \mathrm{kg}$ ) -Electrical power and direct heating prospects.

High to very high enthalpy ranking (4-5) was calculated for the deepest portions of the WCSB (Appendix C Tables A1-A3). The highest ranked prospect area (rank 5) occurs in the northern and western parts of the WCSB, in the Hinton area west of Edson, Alberta, and in Fort Liard NWT. High enthalpy (4) is found in central-western Alberta (municipalities of Grand Prairie, Whitecourt, Wetaskiwin, Lacombe, Red Deer, Blackfalds, Panoka, Rocky Mountain House, Penhold, Devon, and Drayton Valley), north eastern BC (Dawson Creek, Fort St. John, and Fort Nelson) and southeastern Saskatchewan (Estevan area). Medium enthalpy (Ranked 3) geothermal heating is most common in the $2-3 \mathrm{~km}$ deep parts of the basin in Alberta and southern Saskatchewan. In the shallow parts of the basin (see Tables A4 and A5, lifting of fluid temperature would be needed before direct heating applications could be considered (lifting fluid temperature by heat exchanger from 40 to $50{ }^{\circ} \mathrm{C}$ to at least $70{ }^{\circ} \mathrm{C}$, and or heat pumps would be required).

\section{Conclusions}

The economics of two well systems-producer and reinjection-will depend on drilling cost (increasing exponentially with depth), efficiency of the geothermal power plants (usually very low $10+/-3 \%$ ), or of heat exchangers ( $90 \%$ ) for geothermal heating. The electrical power required for pumps for moving fluids through a two-well system (producer and injector wells) and moving fluids through surface piping and the geothermal plant, has been assessed to vary between 0.1 and $0.7 \mathrm{MW}$ electrical [11]. In the WCSB the required drilling depth to get $>70{ }^{\circ} \mathrm{C}$ resources is on average $2 \mathrm{~km}$, and this increases to $>3.7 \mathrm{~km}$ for electric power at $>120^{\circ} \mathrm{C}$ (Figure 8 ).

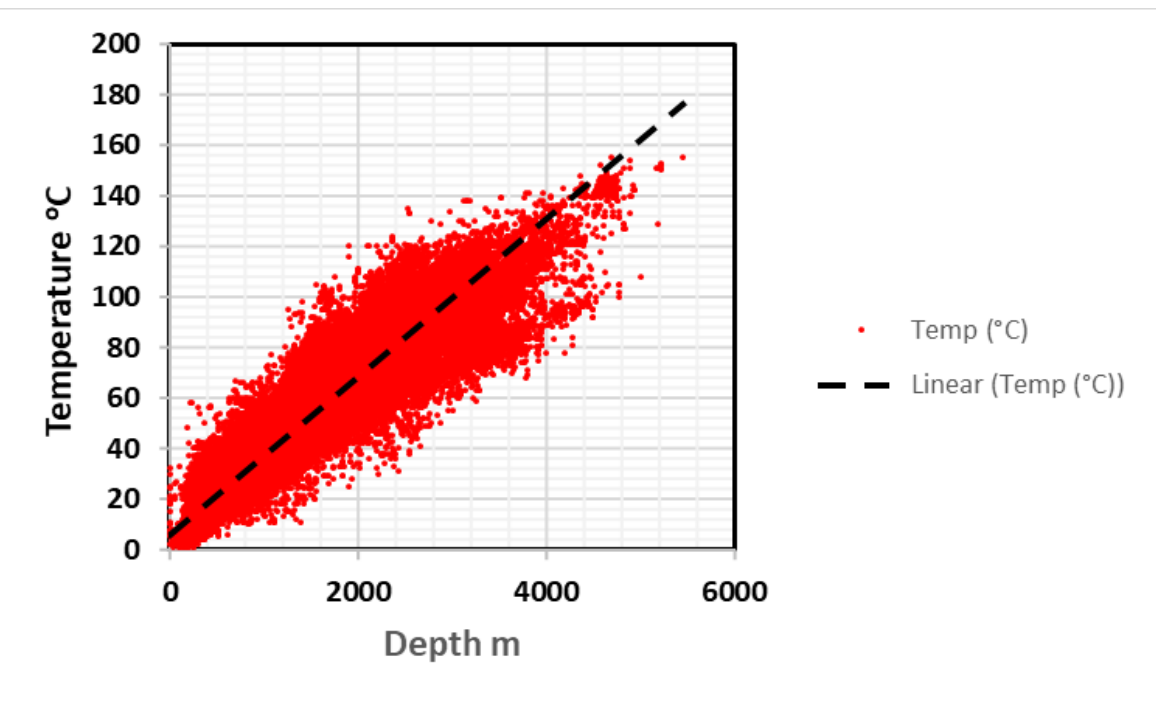

Figure 8. WCSB temperature records from (APP) Annual Pool Pressure test temperature data from shut-in wells. Linear approximation is: $\mathrm{T}\left({ }^{\circ} \mathrm{C}\right)=0.031 \mathrm{z}+5.58$. Correlation coefficient is $\mathrm{R}=0.96$. 
The costs of drilling geothermal wells can be calculated from the equation given by Lukawski et al. [44], with a correlation coefficient of 0.92 :

$$
\text { Geothermal well cost }=1.72 \times 10^{-7} \times(z)^{2}+2.3 \times 10^{-3} \times z-0.62
$$

where cost is in (\$) and $z(m)$ is depth of well. The geothermal wells drilling cost is higher than these of oil and gas [43].

In Tables A1-A5 in Appendix C we gave depths to be drilled to prospective geothermal formations. There are few communities with good prospects at depths less than $2 \mathrm{~km}$. Drilling a well to $2-3 \mathrm{~km}$ (see depth to drill against geological cross section WCSB in Figure 2) would be expensive, $\$ 4.7$ to $8 \mathrm{~m}$ per borehole, and two wells are needed for a doublet geothermal system. Geothermal electrical power production by low efficiency (some $10 \%+/-3 \%$ ) geothermal power plants requires $>120{ }^{\circ} \mathrm{C}$ and preferably $>150{ }^{\circ} \mathrm{C}$. Such projects would require $4-5 \mathrm{~km}$ drilling depth costing $\$ 11-15 \mathrm{~m}$, respectively. This means usually that the drilling cost per one MW electrical would be some $\$ 15 \mathrm{~m}$ for the best cases we show in Tables A1-A3. This high drilling cost limits prospects for economic geothermal electrical power to best case scenarios under current drilling technology. Such high cost per $\mathrm{MW}_{\text {electrical }}$ would not be competitive with wind that has a typical cost of \$3-5 million per megawatt (MW) of electricity-producing capacity. However, geothermal provides baseload power supply that may make the higher cost for reliable generation more attractive.

Municipalities with potentiall for heat energy production, heating $>1 \mathrm{k}$ households, are highlighted in Appendix C Tables A1-A5. These are recommended to be explored first. Large areas of the WCSB are outside the prospects for deep heat for direct heating. These are in many cases areas with temperatures that are still suitable for low enthalpy geothermal heat use. They are temperature $<60{ }^{\circ} \mathrm{C}$ and shallower depths for drilling $(<2 \mathrm{~km})$. Lower enthalpy geothermal sites would be still good for geothermal heating using non-direct techniques, including heat pumps for the lowest enthalpy shallow basin locations. Heat pumps require external energy like electrical power which would require connection with other renewables. Geothermal heating greenhouses could be an opportunity to be explored next.

We showed that direct heating by geothermal energy is most promising for deeper parts of the WCSB in the central and western parts of Alberta, northeastern British Columbia, southern Saskatchewan, and southwestern NWT, where $>70^{\circ} \mathrm{C}$ aquifers with the prospect of $>30 \mathrm{~kg} / \mathrm{s}$ production rates are feasible. Power production is possible near only a limited number of communities, including the Hinton-Edson-Grand Prairie area in Alberta, Fort Liard in southwestern NWT, and Weyburn-Estevan in southern Saskatchewan. Electrical power from geothermal is between single decimals of MW electrical and up to $3 \mathrm{MW}$ electrical (see our calculations in Table A1) assuming a maximum flow rates of $80 \mathrm{~kg} / \mathrm{s}$. However, horizontal wells in target horizons promise to increase potential production rates that could make power production more viable in areas of suitable temperatures as in the ongoing DEEP project [45-48].

The above calculations of heat and electrical power that is feasible to produce from geothermal sources are first order estimates due to uncertainty in production rates. There are several approaches we used to assess reasonable production rates. We took the most probable range of required flow rates $(30-80 \mathrm{Kg} / \mathrm{s})$ based on real pumping test data $[11,35]$, estimates based on hydraulic head and permeability data [37,38,42], as well as examination of thousands of pumping and reinjecting tests through the WCSB [35]. Fluid production rates required to achieve different levels of energy production were calculated by others, $[49,50]$. To produce $1 \mathrm{MW}_{\text {electric }}$ the flow rate should be from 30 to $60 \mathrm{~kg} / \mathrm{s}$ for the northwestern British Columbia part of the WCSB, according to Palmer-Wilson et al. [50]. It makes good sense if the geothermal two-well systems are to be engineered by EGS, to enhance permeability and flow [5].

Further research is recommended that focuses on the geothermal potential of specific municipalities based on more in-depth analyses of local geothermal and geologic conditions. 
In particular, aquifer parameters require further study given their heterogeneous nature, making it difficult to predict local hydrogeological properties.

Author Contributions: Conceptualization, J.M. and S.E.G.; methodology, J.M.; software, J.M.; validation, J.M., S.E.G.; formal analysis, J.M.; investigation, J.M.; resources, J.M.; data curation, J.M.; writing —original draft preparation, J.M.; writing - review and editing, S.E.G.; visualization, J.M.; supervision, S.E.G.; project administration, S.E.G.; funding acquisition, S.E.G. All authors have read and agreed to the published version of the manuscript.

Funding: This research received no external funding.

Institutional Review Board Statement: Not applicable.

Informed Consent Statement: Not applicable.

Data Availability Statement: Data in Appendix A Tables will be available upon request from the first author.

Acknowledgments: We would like to thank three anonymous reviewers for their useful comments. We acknowledge GSC Calgary for support. First Author acknowledge Helmholtz Alberta Initiative University of Alberta project and especially to colleagues: Martyn Unsworth, Simon Weides, Greg Nieuwenhuis; and Tibor Lengyel whose cooperation was essential.

Conflicts of Interest: The authors declare no conflict of interest.

\section{Appendix A}

Saskatchewan SE Manitoba municipalities $>3 \mathrm{k}$ population against maps of geothermal energy prospects for communities $>3 \mathrm{k}$.

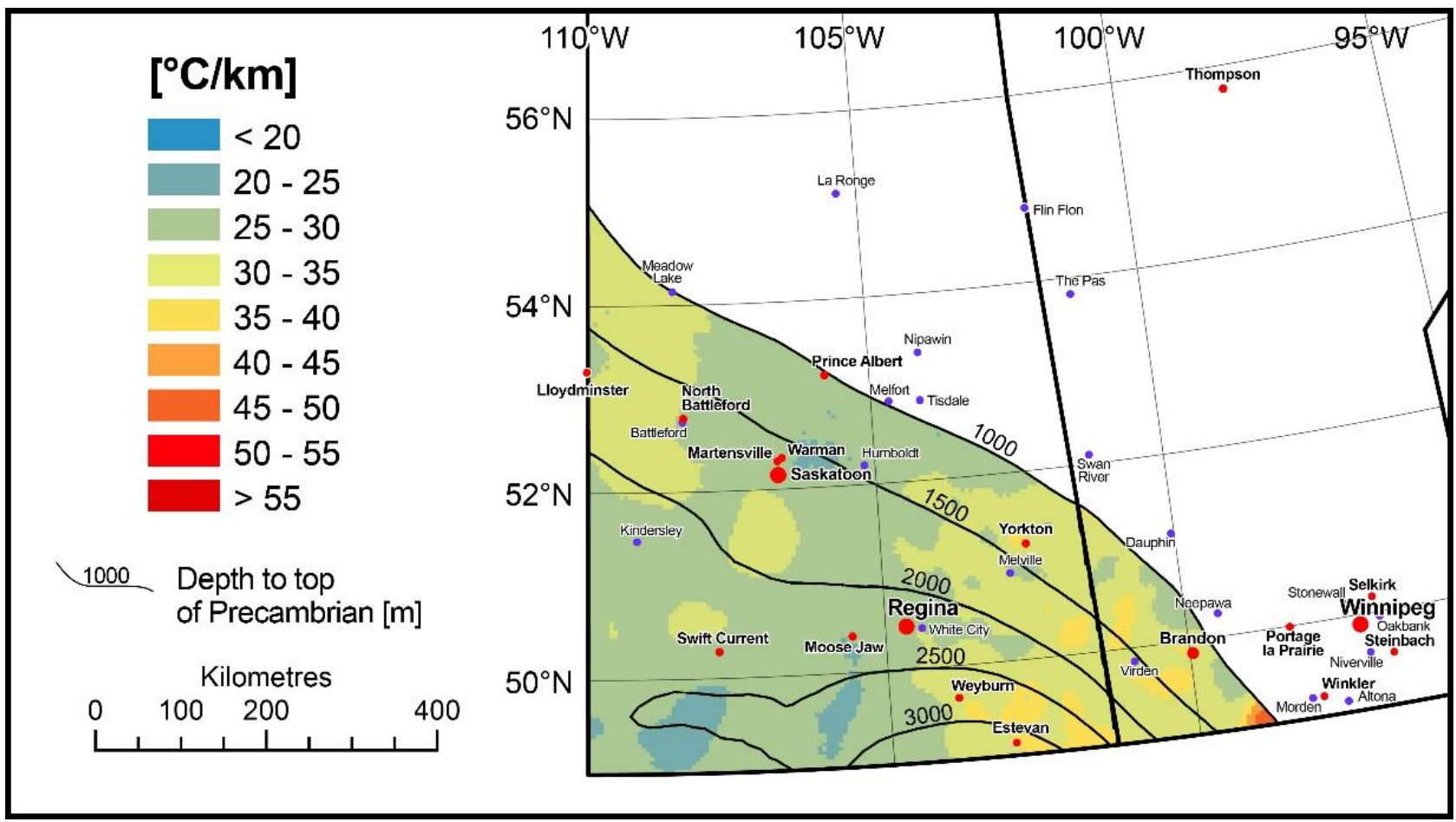

Figure A1. Geothermal gradient-Saskatchewan-Manitoba WCSB. 


\section{$\left[{ }^{\circ} \mathrm{C}\right]$}

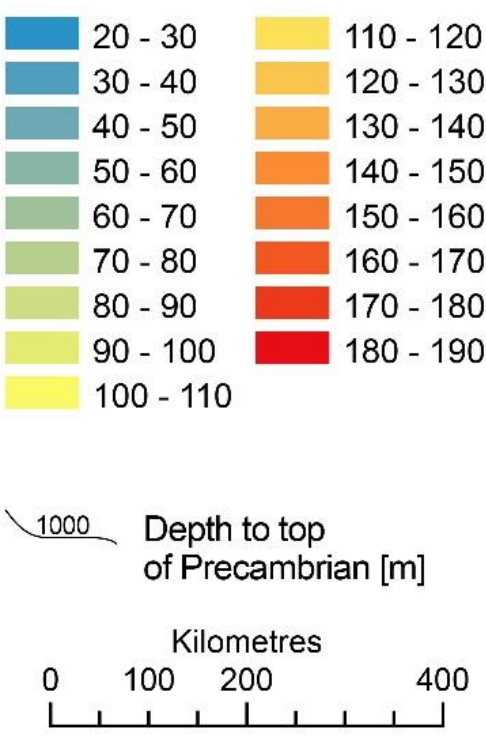

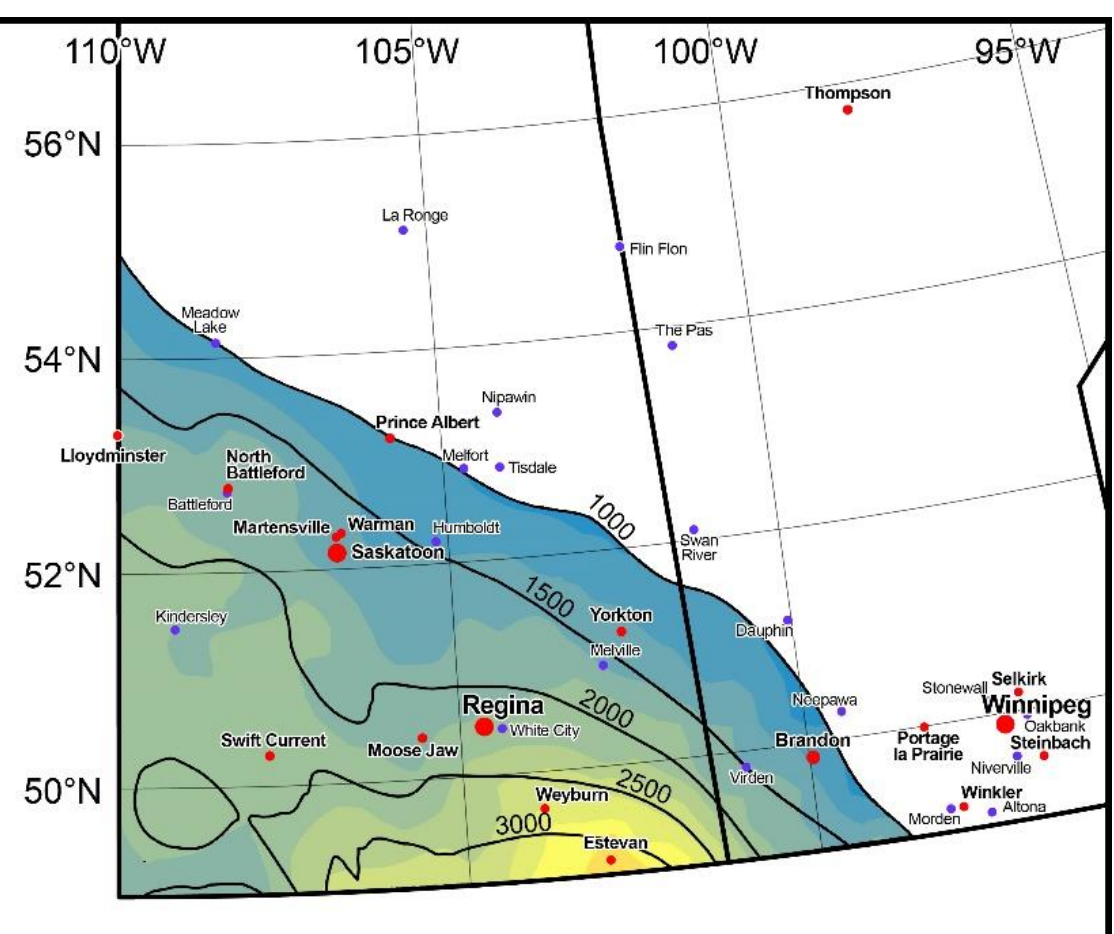

Figure A2. Temperatures top of Precambrian-Saskatchewan-Manitoba WCSB.

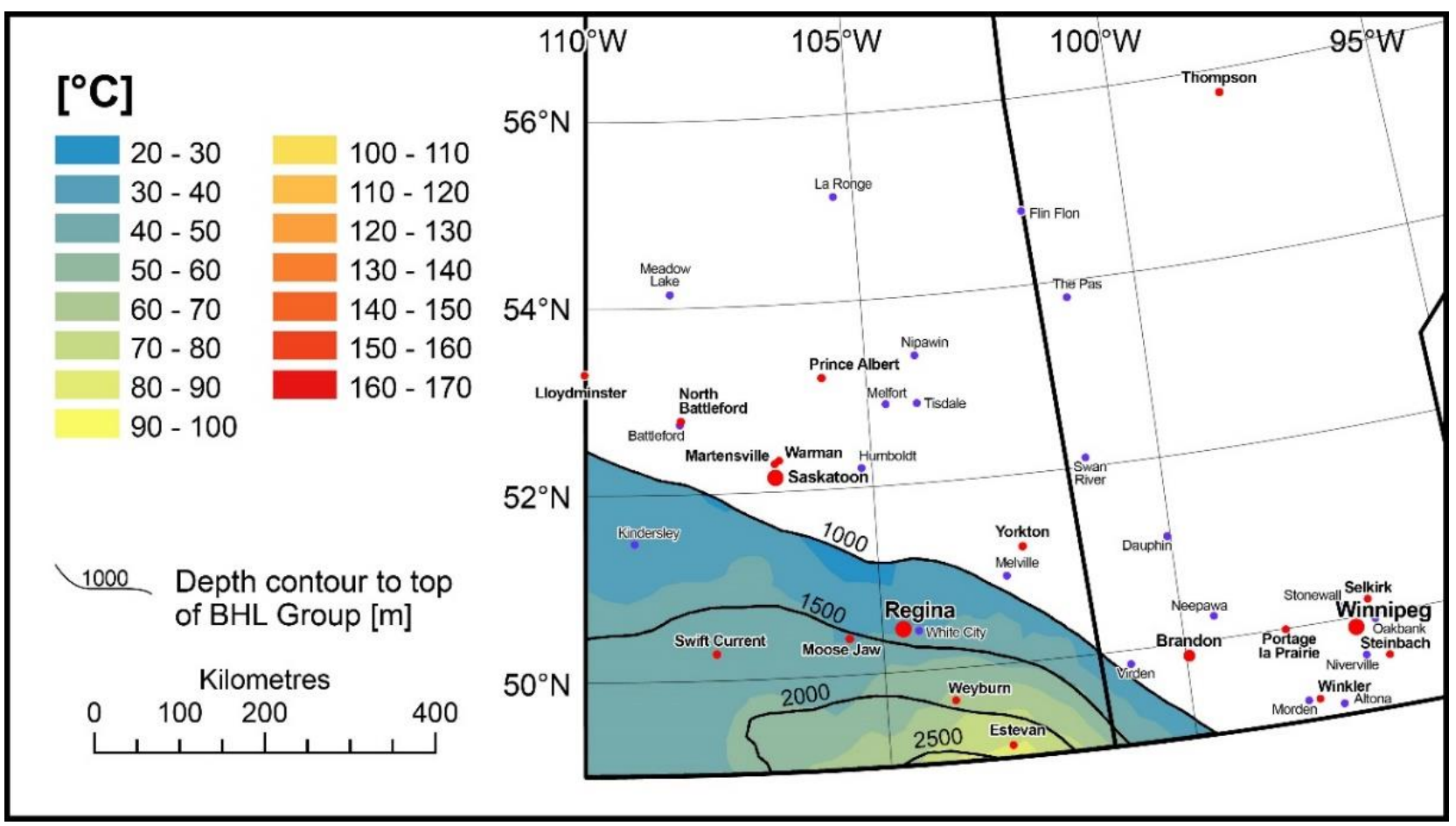

Figure A3. Temperatures top of BHL Group—Saskatchewan-Manitoba WCSB. 


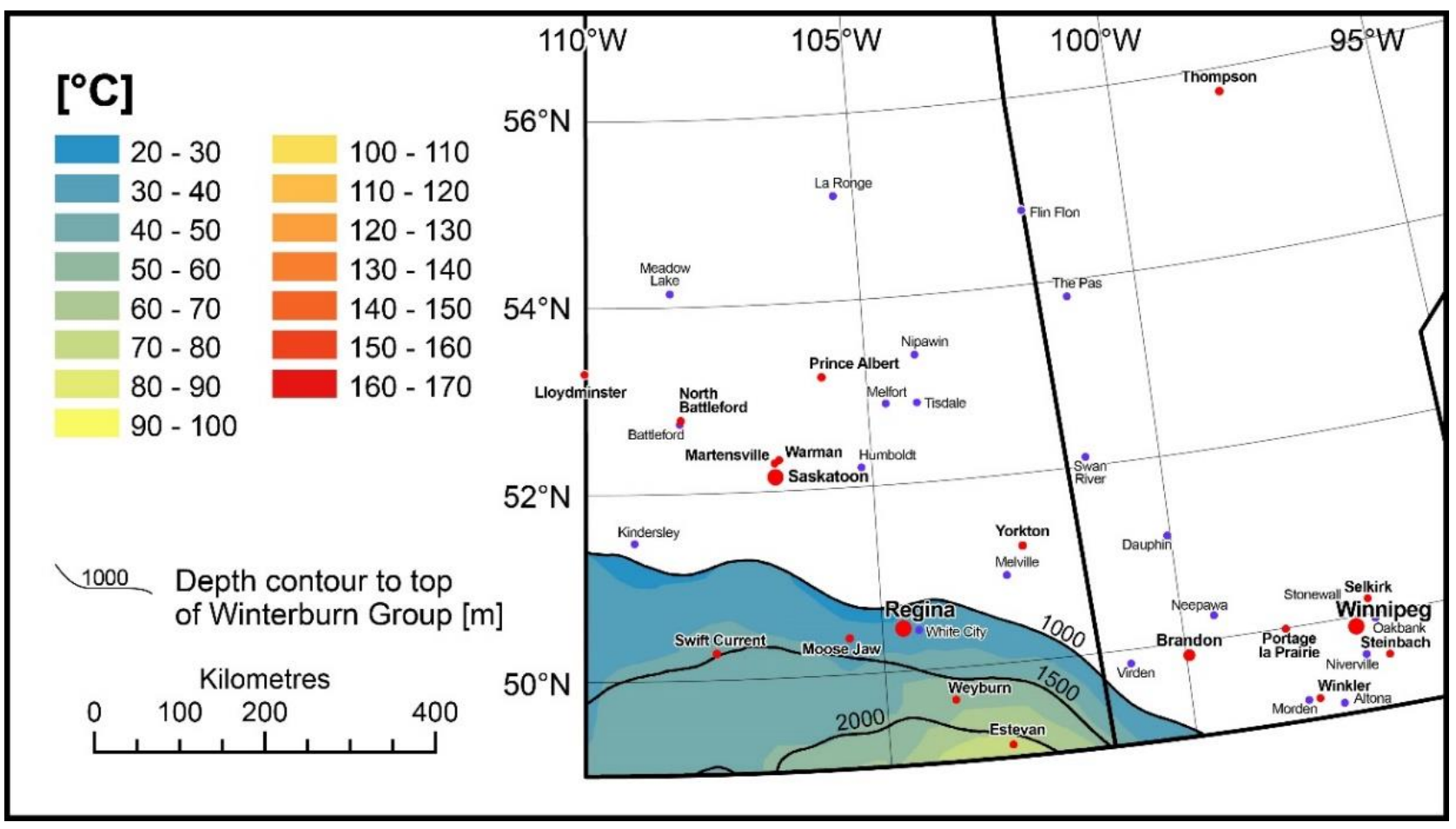

Figure A4. Temperatures top of Winterburn Group—Saskatchewan-Manitoba WCSB.

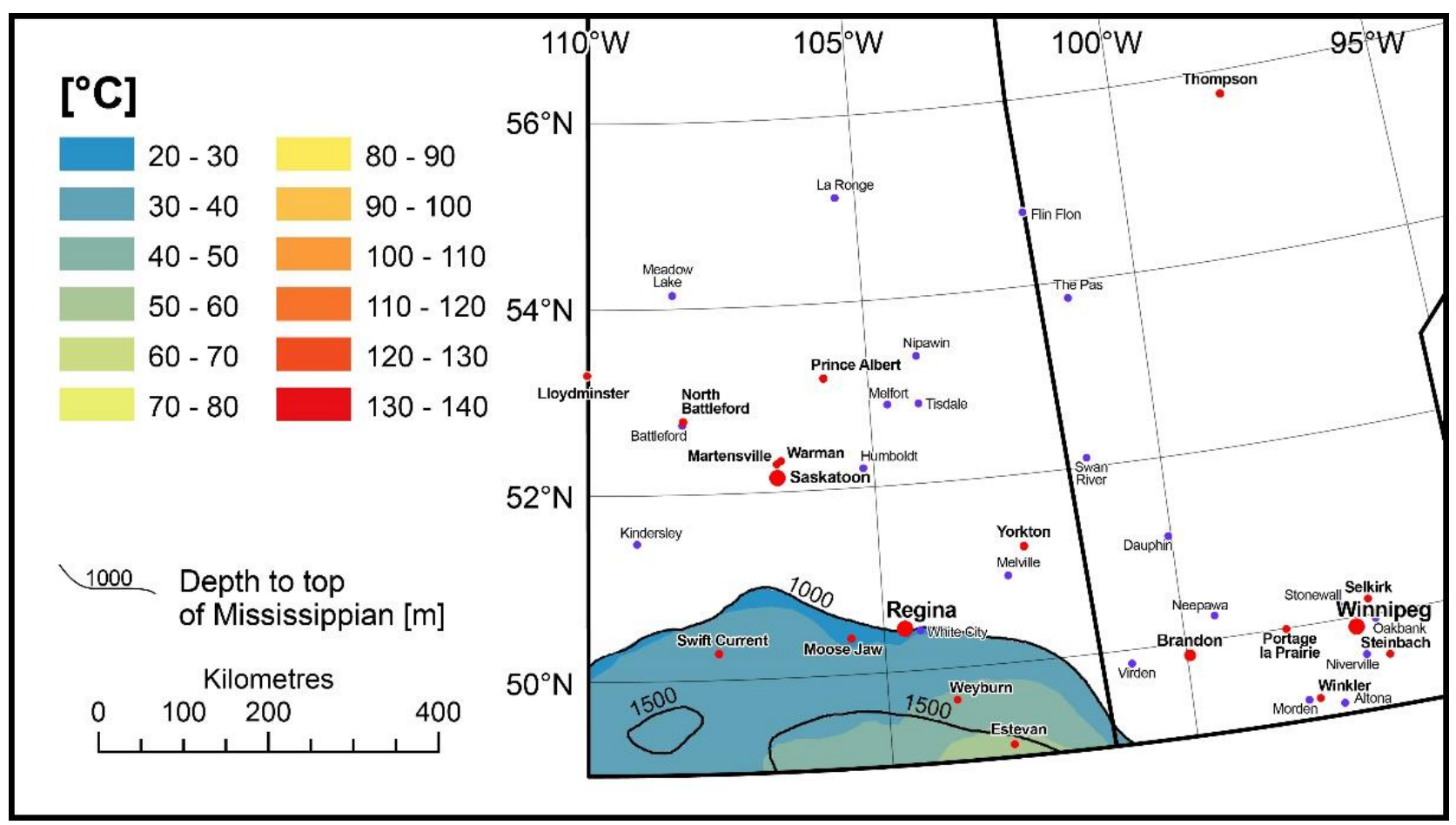

Figure A5. Temperatures top of Mississippian-Saskatchewan-Manitoba WCSB. 


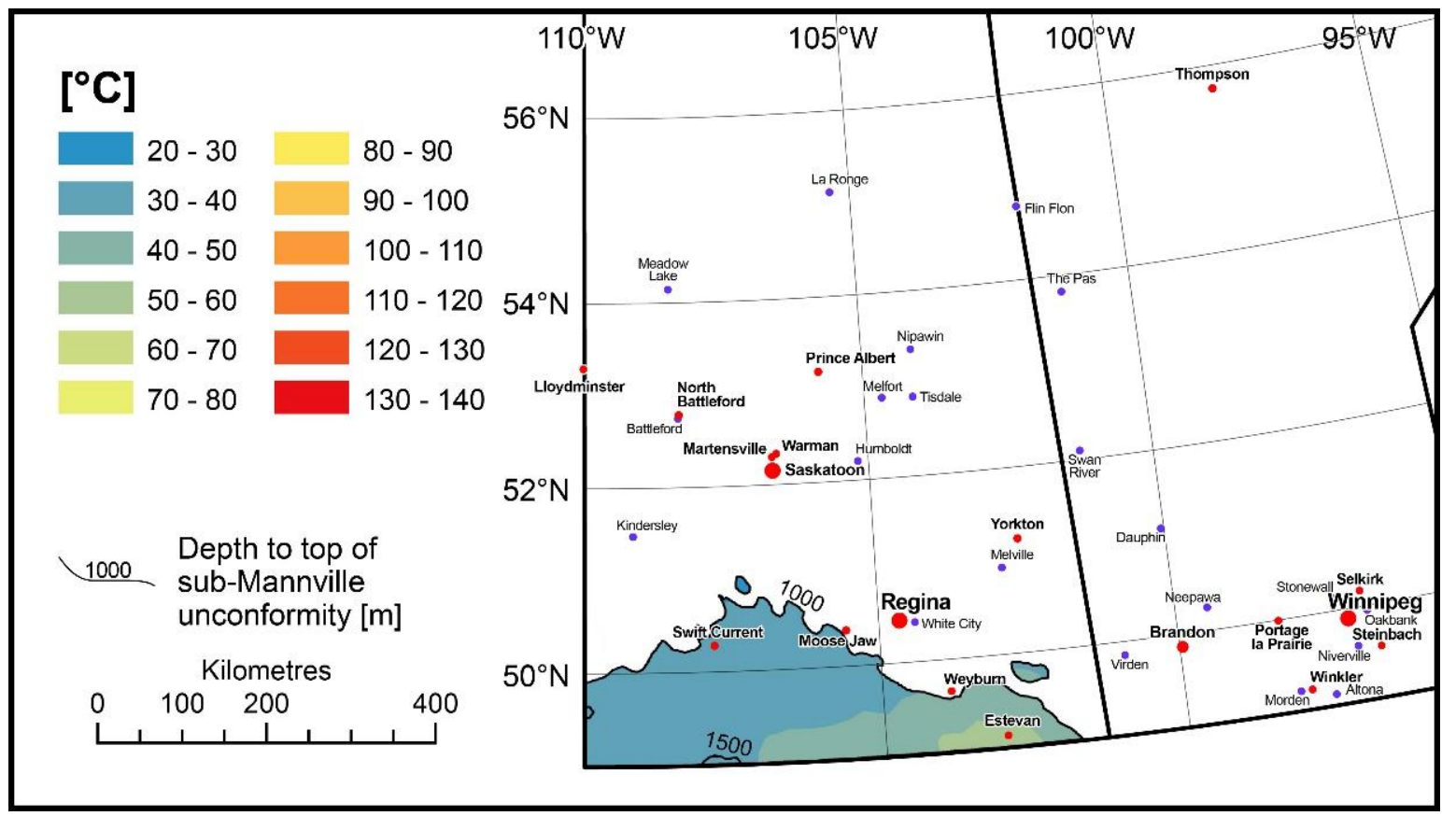

Figure A6. Temperatures top of sub-Mannville unconformity-Saskatchewan-Manitoba WCSB.

\section{Appendix B}

NE British Columbia S. NWT geothermal prospects for communities $>3 \mathrm{k}$.

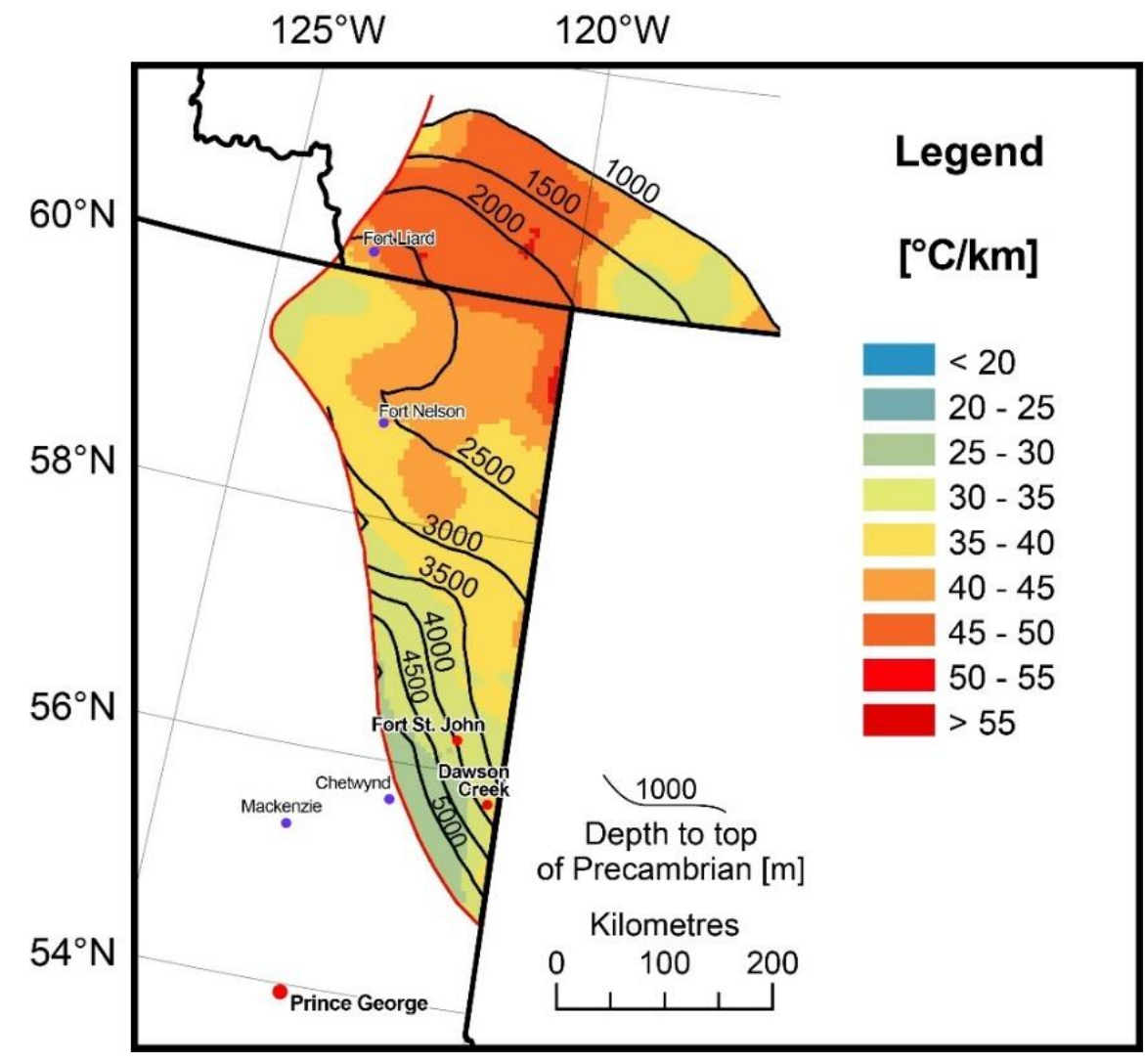

Figure A7. Geothermal gradient-NE British Columbia S. NWT WCSB. 


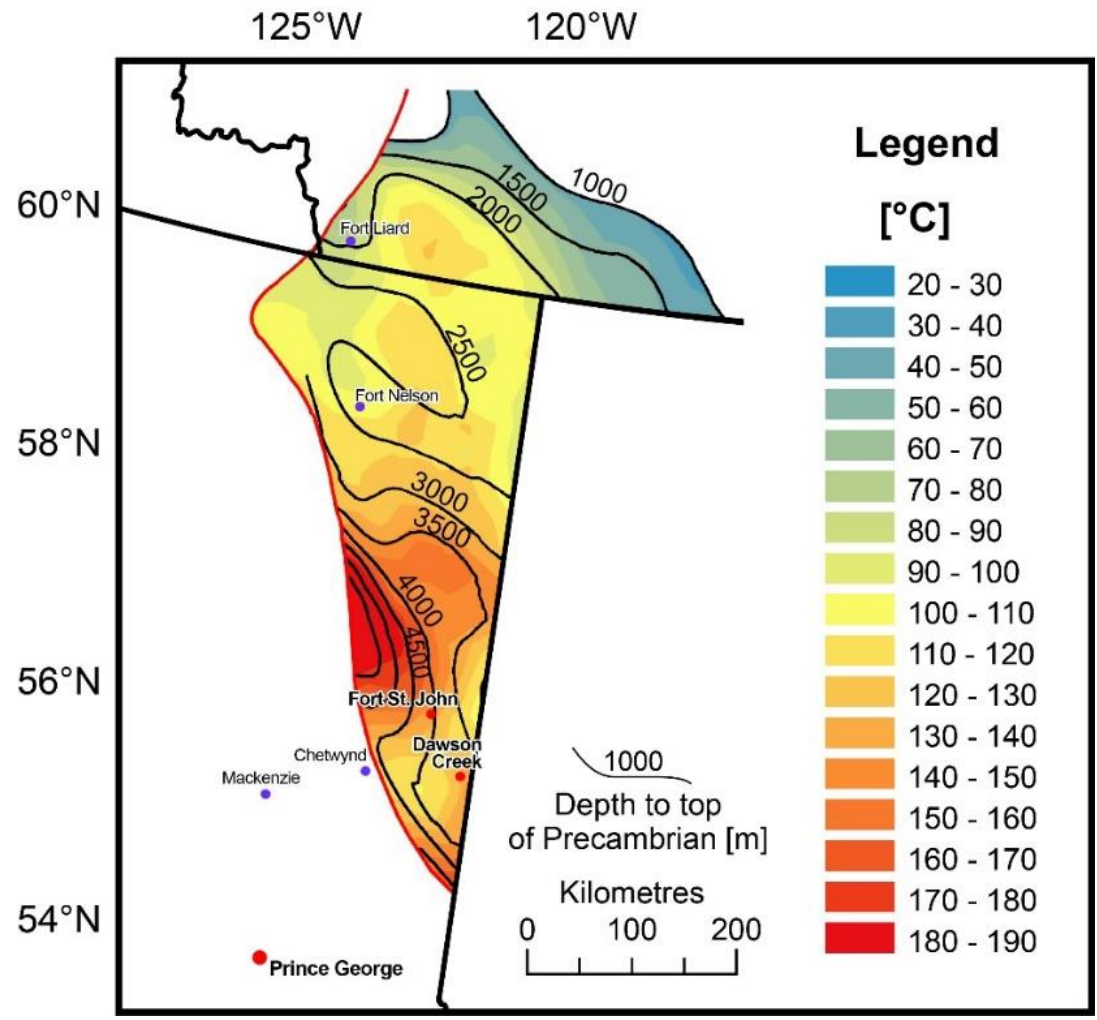

Figure A8. Temperatures top of Precambrian-NE British Columbia S. NWT WCSB.

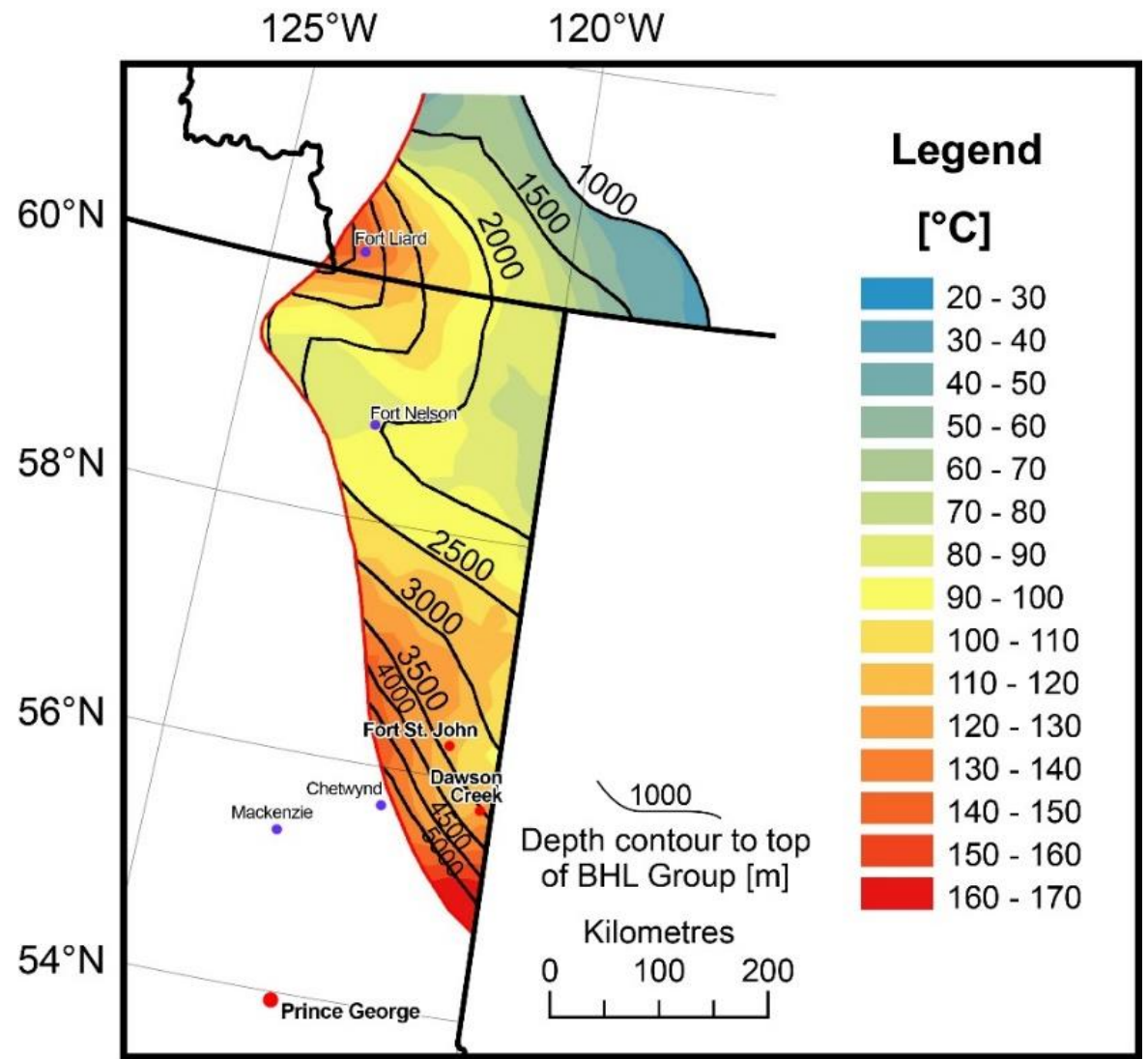

Figure A9. Temperatures top of BHL Group-NE British Columbia S. NWT WCSB 




Figure A10. Temperatures top Winterburn Group-NE British Columbia S. NWT WCSB.

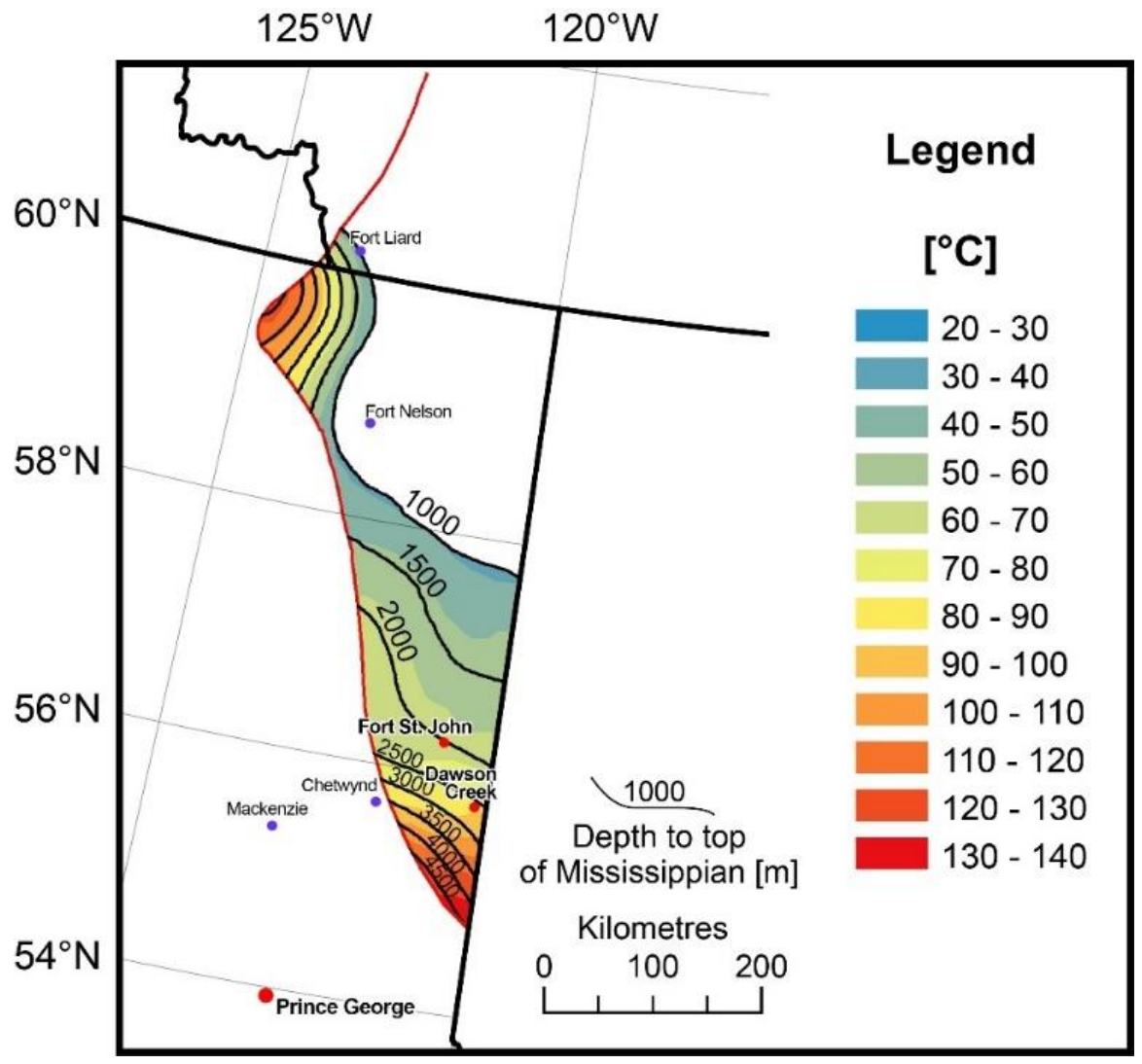

Figure A11. Temperatures top of Mississippian-NE British Columbia S. NWT WCSB. 


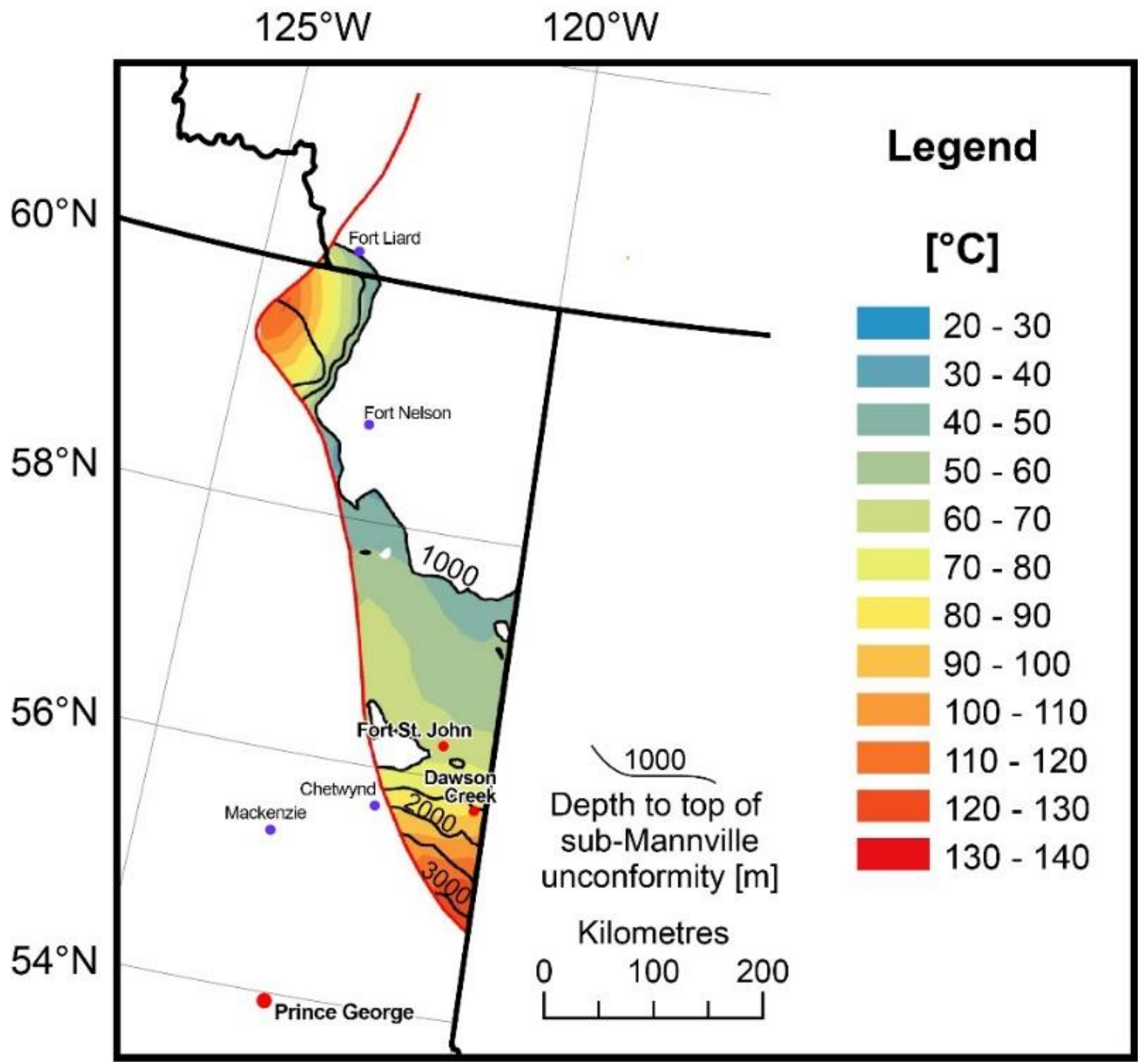

Figure A12. Temperatures top of sub-Mannville Unconformity-NE British Columbia S. NWT WCSB.

\section{Appendix C}

Tables of results.

The results of calculations for all $>3 \mathrm{k}$ population cities and towns in the WCSB are shown in Tables A1-A5. These Tables show geothermal energy for a range of flow rates (well production rate), specific heat capacity of geothermal brine, the number of direct geothermal heated households feasible for small communities of $>3 \mathrm{k}$ to $<10 \mathrm{k}$ population at average energy use of $130 \mathrm{GJ} /$ Year at 0.7 yearly uses, enthalpy, and calculated formation temperature and depth required to drill to prospective geological formations at these calculated temperatures.

The best opportunities for geothermal heat use are highlighted in yellow and geothermal power most prospects are highlighted in red. 


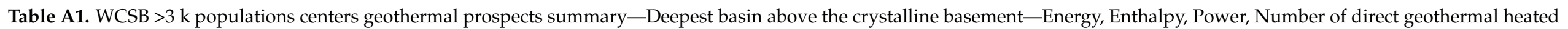
Energy, Enthalpy, Power, No. of direct deep geothermal energy heated households feasible.

\begin{tabular}{|c|c|c|c|c|c|c|c|c|c|c|c|c|c|}
\hline City Location & Province & Temperature $T$ & Energy 1 & Energy 2 & Energy 3 & Energy 4 & Households & Households & Power & Power & $\begin{array}{l}\text { Enthalpy } \\
\text { Gain }\end{array}$ & Formation Group & Depth \\
\hline & & & $\begin{array}{l}\text { at } C=3993 \\
\text { at } 30 \mathrm{~kg} / \mathrm{s}\end{array}$ & $\begin{array}{l}\text { at } C=3150 \\
\text { at } 30 \mathrm{~kg} / \mathrm{s}\end{array}$ & $\begin{array}{l}\text { at } C=3993 \\
\text { at } 80 \mathrm{~kg} / \mathrm{s}\end{array}$ & $\begin{array}{l}\text { at } C=3150 \\
\text { at } 80 \mathrm{~kg} / \mathrm{s}\end{array}$ & $\begin{array}{l}\text { Minimum } \\
\text { number }\end{array}$ & $\begin{array}{l}\text { Maximum } \\
\text { number }\end{array}$ & $\begin{array}{l}\text { at } C=3993 \\
\text { at } 30 \mathrm{~kg} / \mathrm{s}\end{array}$ & $\begin{array}{l}\text { at } C=3150 \\
\text { at } 80 \mathrm{~kg} / \mathrm{s}\end{array}$ & at $\mathrm{C}=3993$ & & \\
\hline Name & Name & ${ }^{\circ} \mathrm{C}$ & GJ Year & GJ Year & GJ Year & GJ Year & $\begin{array}{l}\text { @ 130 } \\
\text { GJ/Year }\end{array}$ & $\begin{array}{l}\text { @ 130 } \\
\text { GJ/Year }\end{array}$ & MW el. & MW el. & $\mathrm{kJ} / \mathrm{kg}$ & & $\mathrm{km}$ \\
\hline Airdrie & $\mathrm{AB}$ & 90 & 66,110 & 52,153 & 176,293 & 139,074 & 401 & 1356 & 0.5 & 0.8 & 280 & $\begin{array}{l}\text { M. Cambrian } \\
\text { Basal Sands }\end{array}$ & 3.7 \\
\hline Banff & $\mathrm{AB}$ & $?$ & - & - & _- & _ & - & - & _- & _- & _ & Disturbed belt & $?$ \\
\hline Barrhead & $\mathrm{AB}$ & 83.2 & 51,125 & 40,331 & 136,333 & 107,550 & 310 & 1049 & 0.4 & 0.6 & 252 & $\begin{array}{l}\text { M. Cambrian } \\
\text { Basal Sands. }\end{array}$ & 2.6 \\
\hline Battleford & Sask. & 51 & - & - & - & - & - & - & - & - & 124 & $\begin{array}{l}\text { M. Cambrian } \\
\text { Basal Sands. }\end{array}$ & 1.7 \\
\hline Beaumont & $\mathrm{AB}$ & 90 & 66,110 & 52,153 & 176,293 & 139,074 & 401 & 1356 & 0.5 & 0.8 & 280 & $\begin{array}{l}\text { M. Cambrian } \\
\text { Basal Sands. }\end{array}$ & 2.6 \\
\hline Blackfalds & $\mathrm{AB}$ & 120.25 & 132,770 & 104,740 & 354,054 & 279,306 & 806 & 2723 & 0.8 & 1.5 & 400 & $\begin{array}{l}\text { M. Cambrian } \\
\text { Basal Sands. }\end{array}$ & 3.25 \\
\hline Bonnyville & $\mathrm{AB}$ & 49 & - & - & - & - & - & - & - & - & 116 & $\begin{array}{l}\text { M. Cambrian } \\
\text { Basal Sands. }\end{array}$ & 1.4 \\
\hline Brooks & $A B$ & 70 & 22,037 & 17,384 & 58,764 & 46,358 & 134 & 452 & 0.2 & 0.3 & 200 & $\begin{array}{l}\text { M. Cambrian } \\
\text { Basal Sands. }\end{array}$ & 2.4 \\
\hline Calgary & $\mathrm{AB}$ & 100 & 88,146 & 69,537 & 235,057 & 185,432 & 535 & 1808 & 0.6 & 1.0 & 319 & $\begin{array}{l}\text { M. Cambrian } \\
\text { Basal Sands. }\end{array}$ & 3.8 \\
\hline Camrose & $\mathrm{AB}$ & 80 & 44,073 & 34,768 & 117,528 & 92,716 & 267 & 904 & 0.4 & 0.5 & 240 & $\begin{array}{l}\text { M. Cambrian } \\
\text { Basal Sands. }\end{array}$ & 2.4 \\
\hline $\begin{array}{l}\text { Canmore } \\
\text { Cardston }\end{array}$ & $\begin{array}{l}\mathrm{AB} \\
\mathrm{AB}\end{array}$ & $\begin{array}{l}40 \\
70\end{array}$ & 22,037 & $\overline{17}, 384$ & $\overline{5} 8,764$ & 46,358 & 134 & 452 & (7) & 03 & 80 & 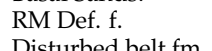 & $\overline{3} 5$ \\
\hline Carstairs & $\mathrm{AB}$ & 90 & 66,110 & 52,153 & 176,293 & $\begin{array}{l}40,030 \\
139,074\end{array}$ & 401 & 1356 & 0.5 & 0.8 & 280 & $\begin{array}{l}\text { M. Cambrian } \\
\text { Basal Sands. }\end{array}$ & 3.6 \\
\hline Chestermere & $\mathrm{AB}$ & 90 & 66,110 & 52,153 & 176,293 & 139,074 & 401 & 1356 & 0.5 & 0.8 & 280 & $\begin{array}{l}\text { Basal Sands. } \\
\text { M. Cambrian } \\
\text { Basal Sands. }\end{array}$ & 3.7 \\
\hline Claresholm & $\mathrm{AB}$ & 75.6 & 34,377 & 27,119 & 91,672 & 72,318 & 209 & 705 & 0.3 & 0.4 & 222 & $\begin{array}{l}\text { M. Cambrian } \\
\text { Basal Sands. }\end{array}$ & 3.6 \\
\hline Coaldale & $\mathrm{AB}$ & 62.5 & 5509 & 4346 & 14,691 & 11,589 & 33 & 113 & 0.1 & 0.1 & 170 & $\begin{array}{l}\text { M. Cambrian } \\
\text { Basal Sands. }\end{array}$ & 2.5 \\
\hline Cochrane & $\mathrm{AB}$ & 100 & 88,146 & 69,537 & 235,057 & 185,432 & 535 & 1808 & 0.6 & 1.0 & 319 & $\begin{array}{l}\text { M. Cambrian } \\
\text { Basal Sands. }\end{array}$ & 4.1 \\
\hline Cold Lake & $\mathrm{AB}$ & 42 & - & - & - & - & - & - & - & - & 88 & $\begin{array}{l}\text { M. Cambrian } \\
\text { Basal Sands. }\end{array}$ & 1.2 \\
\hline Cold Lake & $\mathrm{AB}$ & 40 & - & - & - & - & - & - & - & - & 80 & $\begin{array}{l}\text { M. Cambrian } \\
\text { Basal Sands. }\end{array}$ & 1.2 \\
\hline Dawson Creek & NE BC & 137 & 168,580 & 132,989 & 449,546 & 354,638 & 1023 & 3458 & 1.0 & 1.9 & 465 & $\begin{array}{l}\text { M. Cambrian } \\
\text { Basal Sands. }\end{array}$ & 3.9 \\
\hline Devon & $\mathrm{AB}$ & 99.9 & 87,926 & 69,363 & 234,469 & 184,968 & 534 & 1804 & 0.6 & 1.0 & 319 & $\begin{array}{l}\text { M. Cambrian } \\
\text { Basal Sands. }\end{array}$ & 2.7 \\
\hline Didsbury & $\mathrm{AB}$ & 92.5 & 71,619 & 56,499 & 190,984 & 150,663 & 435 & 1469 & 0.5 & 0.8 & 289 & $\begin{array}{l}\text { M. Cambrian } \\
\text { Basal Sands. }\end{array}$ & 3.7 \\
\hline Drayton Valley & $\mathrm{AB}$ & 119 & 130,016 & 102,567 & 346,709 & 273,512 & 789 & 2667 & 0.8 & 1.5 & 395 & $\begin{array}{l}\text { M. Cambrian } \\
\text { Basal Sands. }\end{array}$ & 3.4 \\
\hline
\end{tabular}


Table A1. Cont.

\begin{tabular}{|c|c|c|c|c|c|c|c|c|c|c|c|c|c|}
\hline City Location & Province & Temperature $\mathrm{T}$ & Energy 1 & Energy 2 & Energy 3 & Energy 4 & Households & Households & Power & Power & $\begin{array}{l}\text { Enthalpy } \\
\text { Gain }\end{array}$ & Formation Group & Depth \\
\hline & & & $\begin{array}{l}\text { at } C=3993 \\
\text { at } 30 \mathrm{~kg} / \mathrm{s}\end{array}$ & $\begin{array}{l}\text { at } C=3150 \\
\text { at } 30 \mathrm{~kg} / \mathrm{s}\end{array}$ & $\begin{array}{l}\text { at } C=3993 \\
\text { at } 80 \mathrm{~kg} / \mathrm{s}\end{array}$ & $\begin{array}{l}\text { at } C=3150 \\
\text { at } 80 \mathrm{~kg} / \mathrm{s}\end{array}$ & $\begin{array}{l}\text { Minimum } \\
\text { number }\end{array}$ & $\begin{array}{l}\text { Maximum } \\
\text { number }\end{array}$ & $\begin{array}{l}\text { at } C=3993 \\
\text { at } 30 \mathrm{~kg} / \mathrm{s}\end{array}$ & $\begin{array}{l}\text { at } C=3150 \\
\text { at } 80 \mathrm{~kg} / \mathrm{s}\end{array}$ & at $C=3993$ & & \\
\hline Name & Name & ${ }^{\circ} \mathrm{C}$ & GJ Year & GJ Year & GJ Year & GJ Year & $\begin{array}{l}\text { @ } 130 \\
\text { GJ/Year }\end{array}$ & $\begin{array}{l}@ 130 \\
\text { GJ/Year }\end{array}$ & MW el. & MW el. & $\mathrm{kJ} / \mathrm{kg}$ & & $\mathrm{km}$ \\
\hline Drumheller & $\mathrm{AB}$ & 72.9 & 28,427 & 22,426 & 75,806 & 59,802 & 173 & 583 & 0.3 & 0.3 & 211 & $\begin{array}{l}\text { M. Cambrian } \\
\text { Basal Sands. }\end{array}$ & 2.7 \\
\hline E. Lloydminster & Sask. & 51 & - & - & - & - & - & - & - & - & 124 & $\begin{array}{l}\text { M. Cambrian } \\
\text { Basal Sands. }\end{array}$ & 1.7 \\
\hline Edmonton & $\mathrm{AB}$ & 90 & 66,110 & 52,153 & 176,293 & 139,074 & 401 & 1356 & 0.5 & 0.8 & 280 & $\begin{array}{l}\text { M. Cambrian } \\
\text { Basal Sands. }\end{array}$ & 2.5 \\
\hline Edson & $\mathrm{AB}$ & 147 & 191,718 & 151,243 & 511,248 & 403,314 & 1163 & 3933 & 1.2 & 2.2 & 507 & $\begin{array}{l}\text { M. Cambrian } \\
\text { Basal Sands. }\end{array}$ & 4.2 \\
\hline Estevan & Sask. & 115 & 121,642 & 95,961 & 324,378 & 255,896 & 738 & 2495 & 0.8 & 1.4 & 380 & $\begin{array}{l}\text { U. Cambrian } \\
\text { Deadwood }\end{array}$ & 3.2 \\
\hline Fort Liard & NWT & 170 & 242,402 & 191,226 & 646,406 & 509,937 & 1471 & 4972 & 1.4 & 2.8 & 599 & $\begin{array}{l}\text { M. Cambrian } \\
\text { Basal Sands. }\end{array}$ & 4.25 \\
\hline Fort Nelson & NE BC & 104 & 96,961 & 76,491 & 258,562 & 203,975 & 588 & 1989 & 0.6 & 1.1 & 335 & $\begin{array}{l}\text { M. Cambrian } \\
\text { Basal Sands. }\end{array}$ & 2.6 \\
\hline Fort Sask. & $\mathrm{AB}$ & 90 & 66,110 & 52,153 & 176,293 & 139,074 & 401 & 1356 & 0.5 & 0.8 & 280 & $\begin{array}{l}\text { M. Cambrian } \\
\text { Basal Sands. }\end{array}$ & 2.4 \\
\hline Fort St. John & NE BC & 128 & 149,849 & 118,213 & 399,596 & 315,234 & 909 & 3074 & 0.9 & 1.7 & 431 & $\begin{array}{l}\text { M. Cambrian } \\
\text { Basal Sands. }\end{array}$ & 4 \\
\hline Grand Centre & $\mathrm{AB}$ & 40.5 & - & - & - & _- & - & - & - & - & 82 & $\begin{array}{l}\text { M. Cambrian } \\
\text { Basal Sands. }\end{array}$ & 1.35 \\
\hline $\begin{array}{l}\text { Grande Cache } \\
\text { Grande Prairie }\end{array}$ & $\begin{array}{l}A B \\
A B\end{array}$ & $\begin{array}{l}? \\
140\end{array}$ & $\overline{176,293}$ & $\overline{1} 39,074$ & $\overline{4} 70,113$ & $\overline{3} 70,863$ & 1070 & 3616 & $\overline{1} .1$ & $\overline{2} .0$ & $\overline{4} 79$ & $\begin{array}{l}\text { Disturbed belt fm } \\
\text { Granite Wash }\end{array}$ & $\begin{array}{l}? \\
3.7\end{array}$ \\
\hline High River & $\mathrm{AB}$ & 100 & 88,146 & 69,537 & 235,057 & 185,432 & 535 & 1808 & 0.6 & 1.0 & 319 & $\begin{array}{l}\text { M. Cambrian } \\
\text { Basal Sands. }\end{array}$ & 4 \\
\hline Hinton & $\mathrm{AB}$ & 170 & 242,402 & 191,226 & 646,406 & 509,937 & 1471 & 4972 & 1.4 & 2.8 & 599 & $\begin{array}{l}\text { M. Cambrian } \\
\text { Basal Sands. }\end{array}$ & 5.6 \\
\hline Humboldt & Sask. & 35 & - & - & - & - & - & - & - & - & 60 & $\begin{array}{l}\text { M. Cambrian } \\
\text { Basal Sands. }\end{array}$ & 1.4 \\
\hline Innisfail & $\mathrm{AB}$ & 87.5 & 60,601 & 47,807 & 161,602 & 127,484 & 368 & 1243 & 0.4 & 0.7 & 270 & $\begin{array}{l}\text { M. Cambrian } \\
\text { Basal Sands. }\end{array}$ & 3.5 \\
\hline Jasper & $\mathrm{AB}$ & $?$ & - & - & - & - & - & - & _- & - & - & Disturbed belt & $?$ \\
\hline Kindersley & Sask. & 59 & - & - & - & - & - & - & - & - & 157 & $\begin{array}{l}\text { M. Cambrian } \\
\text { Basal Sands. }\end{array}$ & 2.2 \\
\hline Lacombe & $\mathrm{AB}$ & 120 & 132,219 & 104,305 & 352,585 & 278,148 & 802 & 2712 & 0.8 & 2.0 & 399 & $\begin{array}{l}\text { M. Cambrian } \\
\text { Basal Sands. }\end{array}$ & 3.2 \\
\hline Langdon & $\mathrm{AB}$ & 80.5 & 45,175 & 35,638 & 120,467 & 95,034 & 274 & 927 & 0.4 & 0.5 & 242 & $\begin{array}{l}\text { M. Cambrian } \\
\text { Basal Sands. }\end{array}$ & 3.5 \\
\hline Leduc & $\mathrm{AB}$ & 110 & 110,183 & 86,921 & 293,821 & 231,790 & 669 & 2260 & 0.7 & 2.0 & 359 & $\begin{array}{l}\text { M. Cambrian } \\
\text { Basal Sands. }\end{array}$ & 2.7 \\
\hline Lethbridge & $\mathrm{AB}$ & 70 & 22,037 & 17,384 & 58,764 & 46,358 & 134 & 452 & 0.2 & 0.3 & 200 & $\begin{array}{l}\text { M. Cambrian } \\
\text { Basal Sands. }\end{array}$ & 2.7 \\
\hline Lloydminster & $\mathrm{AB}$ & 50 & - & - & - & - & - & - & - & - & 120 & $\begin{array}{l}\text { M. Cambrian } \\
\text { Basal Sands. }\end{array}$ & 1.7 \\
\hline
\end{tabular}


Table A1. Cont.

\begin{tabular}{|c|c|c|c|c|c|c|c|c|c|c|c|c|c|}
\hline City Location & Province & Temperature $\mathbf{T}$ & Energy 1 & Energy 2 & Energy 3 & Energy 4 & Households & Households & Power & Power & $\begin{array}{l}\text { Enthalpy } \\
\text { Gain }\end{array}$ & Formation Group & Depth \\
\hline & & & $\begin{array}{l}\text { at } C=3993 \\
\text { at } 30 \mathrm{~kg} / \mathrm{s}\end{array}$ & $\begin{array}{l}\text { at } C=3150 \\
\text { at } 30 \mathrm{~kg} / \mathrm{s}\end{array}$ & $\begin{array}{l}\text { at } C=3993 \\
\text { at } 80 \mathrm{~kg} / \mathrm{s}\end{array}$ & $\begin{array}{l}\text { at } C=3150 \\
\text { at } 80 \mathrm{~kg} / \mathrm{s}\end{array}$ & $\begin{array}{l}\text { Minimum } \\
\text { number }\end{array}$ & $\begin{array}{l}\text { Maximum } \\
\text { number }\end{array}$ & $\begin{array}{l}\text { at } C=3993 \\
\text { at } 30 \mathrm{~kg} / \mathrm{s}\end{array}$ & $\begin{array}{l}\text { at } C=3150 \\
\text { at } 80 \mathrm{~kg} / \mathrm{s}\end{array}$ & at $C=3993$ & & \\
\hline Name & Name & ${ }^{\circ} \mathrm{C}$ & GJ Year & GJ Year & GJ Year & GJ Year & $\begin{array}{l}\text { @ 130 } \\
\text { GJ/Year }\end{array}$ & $\begin{array}{l}@ 130 \\
\text { GJ/Year }\end{array}$ & MW el. & MW el. & $\mathrm{kJ} / \mathrm{kg}$ & & $\mathrm{km}$ \\
\hline Malville & Sask. & 48 & - & - & - & - & - & - & - & - & 112 & $\begin{array}{l}\text { M. Cambrian } \\
\text { Basal Sands. }\end{array}$ & 1.6 \\
\hline Martensville & Sask. & 45 & - & - & - & - & - & - & - & - & 98 & $\begin{array}{l}\text { M. Cambrian } \\
\text { Basal Sands. }\end{array}$ & 1.65 \\
\hline Meadow Lake & Sask. & 35 & - & - & - & - & - & - & - & - & 60 & $\begin{array}{l}\text { M. Cambrian } \\
\text { Basal Sands. }\end{array}$ & 1 \\
\hline Medicine Hat & $\mathrm{AB}$ & 60 & - & - & - & - & - & - & 0.1 & - & 160 & $\begin{array}{l}\text { M. Cambrian } \\
\text { Basal Sands. }\end{array}$ & 2.2 \\
\hline Melford & Sask. & 30 & - & - & - & - & - & - & - & - & 40 & $\begin{array}{l}\text { M. Cambrian } \\
\text { Basal Sands. }\end{array}$ & 1 \\
\hline Moose Jaw & Sask. & 55 & - & - & - & - & - & - & 0.1 & - & 140 & $\begin{array}{l}\text { M. Cambrian } \\
\text { Basal Sands. }\end{array}$ & 2.2 \\
\hline Morinville & $\mathrm{AB}$ & 100 & 88,146 & 69,537 & 235,057 & 185,432 & 535 & 1808 & 0.6 & 1.0 & 319 & $\begin{array}{l}\text { M. Cambrian } \\
\text { Basal Sands. }\end{array}$ & 2.4 \\
\hline $\begin{array}{l}\text { North } \\
\text { Battleford }\end{array}$ & Sask. & 50 & - & - & - & - & - & - & - & - & 118 & $\begin{array}{l}\text { M. Cambrian } \\
\text { Basal Sands. }\end{array}$ & 1.65 \\
\hline Olds & $\mathrm{AB}$ & 90 & 66,110 & 52,153 & 176,293 & 139,074 & 401 & 1356 & 0.5 & 0.8 & 280 & $\begin{array}{l}\text { M. Cambrian } \\
\text { Basal Sands. }\end{array}$ & 3.6 \\
\hline Peace River & $\mathrm{AB}$ & 67.2 & 15,866 & 12,517 & 42,310 & 33,378 & 96 & 325 & 0.2 & 0.2 & 188 & $\begin{array}{l}\text { Devonian Granite } \\
\text { Wash }\end{array}$ & 2.1 \\
\hline Penhold & $\mathrm{AB}$ & 95.2 & 77,569 & 61,192 & 206,850 & 163,180 & 471 & 1591 & 0.5 & 0.9 & 300 & $\begin{array}{l}\text { M. Cambrian } \\
\text { Basal Sands. }\end{array}$ & 3.4 \\
\hline Pincher Creek & $\mathrm{AB}$ & 89.3 & 64,567 & 50,936 & 172,179 & 135,829 & 392 & 1324 & 0.5 & 0.7 & 277 & Disturbed belt fm & 4.7 \\
\hline Ponoka & $\mathrm{AB}$ & 123 & 138,830 & 109,521 & 370,214 & 292,055 & 842 & 2848 & 0.9 & 1.6 & 411 & $\begin{array}{l}\text { M. Cambrian } \\
\text { Basal Sands. }\end{array}$ & 3 \\
\hline Prince Albert & Sask. & 30 & - & - & - & - & - & - & - & - & 40 & $\begin{array}{l}\text { M. Cambrian } \\
\text { Basal Sands. }\end{array}$ & 1 \\
\hline Raymond & $\mathrm{AB}$ & 57.5 & - & - & - & - & - & - & - & - & 150 & $\begin{array}{l}\text { M. Cambrian } \\
\text { Basal Sands. }\end{array}$ & 2.5 \\
\hline Red Deer & $\mathrm{AB}$ & 115 & 121,201 & 95,613 & 323,203 & 254,969 & 735 & 2486 & 0.8 & 1.4 & 379 & $\begin{array}{l}\text { M. Cambrian } \\
\text { Basal Sands. }\end{array}$ & 3.3 \\
\hline Redcliff & $\mathrm{AB}$ & 60.5 & 1102 & 869 & 2938 & 2318 & 7 & 23 & 0.1 & 0.0 & 162 & $\begin{array}{l}\text { M. Cambrian } \\
\text { Basal Sands. }\end{array}$ & 2.2 \\
\hline Regina & Sask. & 61 & 1983 & 1565 & 5289 & 4172 & 12 & 41 & 0.1 & 0.0 & 163 & $\begin{array}{l}\text { U. Cambrian } \\
\text { Deadwood }\end{array}$ & 2.1 \\
\hline $\begin{array}{l}\text { Rocky } \\
\text { Mountain } \\
\text { House }\end{array}$ & $\mathrm{AB}$ & 144 & 185,107 & 146,027 & 493,619 & 389,407 & 1123 & 3797 & 1.1 & 2.1 & 495 & $\begin{array}{l}\text { M. Cambrian } \\
\text { Basal Sands. }\end{array}$ & 4.8 \\
\hline Saskatoon & Sask. & 48 & - & - & - & - & - & - & - & - & 110 & $\begin{array}{l}\text { M. Cambrian } \\
\text { Basal Sands. }\end{array}$ & 1.7 \\
\hline Slave Lake & $\mathrm{AB}$ & 73.5 & 29,749 & 23,469 & 79,332 & 62,583 & 181 & 610 & 0.3 & 0.3 & 214 & $\begin{array}{l}\text { M. Cambrian } \\
\text { Basal Sands. }\end{array}$ & 2.1 \\
\hline Spruce Grove & $\mathrm{AB}$ & 90 & 66,110 & 52,153 & 176,293 & 139,074 & 401 & 1356 & 0.5 & 0.8 & 280 & $\begin{array}{l}\text { M. Cambrian } \\
\text { Basal Sands. }\end{array}$ & 2.6 \\
\hline
\end{tabular}


Table A1. Cont.

\begin{tabular}{|c|c|c|c|c|c|c|c|c|c|c|c|c|c|}
\hline City Location & Province & Temperature T & Energy 1 & Energy 2 & Energy 3 & Energy 4 & Households & Households & Power & Power & $\begin{array}{l}\text { Enthalpy } \\
\text { Gain }\end{array}$ & Formation Group & Depth \\
\hline & & & $\begin{array}{l}\text { at } C=3993 \\
\text { at } 30 \mathrm{~kg} / \mathrm{s}\end{array}$ & $\begin{array}{l}\text { at } C=3150 \\
\text { at } 30 \mathrm{~kg} / \mathrm{s}\end{array}$ & $\begin{array}{l}\text { at } C=3993 \\
\text { at } 80 \mathrm{~kg} / \mathrm{s}\end{array}$ & $\begin{array}{l}\text { at } C=3150 \\
\text { at } 80 \mathrm{~kg} / \mathrm{s}\end{array}$ & $\begin{array}{l}\text { Minimum } \\
\text { number }\end{array}$ & $\begin{array}{l}\text { Maximum } \\
\text { number }\end{array}$ & $\begin{array}{l}\text { at } C=3993 \\
\text { at } 30 \mathrm{~kg} / \mathrm{s}\end{array}$ & $\begin{array}{l}\text { at } C=3150 \\
\text { at } 80 \mathrm{~kg} / \mathrm{s}\end{array}$ & at $\mathrm{C}=3993$ & & \\
\hline Name & Name & ${ }^{\circ} \mathrm{C}$ & GJ Year & GJ Year & GJ Year & GJ Year & $\begin{array}{l}\text { @ 130 } \\
\text { GJ/Year }\end{array}$ & $\begin{array}{l}\text { @ 130 } \\
\text { GJ/Year }\end{array}$ & MW el. & MW el. & $\mathrm{kJ} / \mathrm{kg}$ & & km \\
\hline St. Albert & $\mathrm{AB}$ & 90 & 66,110 & 52,153 & 176,293 & 139,074 & 401 & 1356 & 0.5 & 0.8 & 280 & $\begin{array}{l}\text { M. Cambrian } \\
\text { Basal Sands. }\end{array}$ & 2.5 \\
\hline St. Paul & $\mathrm{AB}$ & 55.25 & - & - & - & - & - & - & - & - & 141 & $\begin{array}{l}\text { M. Cambrian } \\
\text { Basal Sands. }\end{array}$ & 1.7 \\
\hline Stettler & $\mathrm{AB}$ & 78.3 & 40,327 & 31,813 & 107,538 & 84,835 & 245 & 827 & 0.3 & 0.5 & 233 & $\begin{array}{l}\text { M. Cambrian } \\
\text { Basal Sands. }\end{array}$ & 2.7 \\
\hline Stony Plain & $\mathrm{AB}$ & 90 & 66,110 & 52,153 & 176,293 & 139,074 & 401 & 1356 & 0.5 & 0.8 & 280 & $\begin{array}{l}\text { M. Cambrian } \\
\text { Basal Sands. }\end{array}$ & 2.6 \\
\hline Swift Current & Sask. & 65 & 10,578 & 8344 & 28,207 & 22,252 & 64 & 217 & 0.2 & 0.1 & 179 & $\begin{array}{l}\text { M. Cambrian } \\
\text { Basal Sands. }\end{array}$ & 2.4 \\
\hline Taber & $\mathrm{AB}$ & 59.4 & - & - & - & - & - & - & - & - & 157 & $\begin{array}{l}\text { M. Cambrian } \\
\text { Basal Sands. }\end{array}$ & 2.2 \\
\hline Three Hills & $\mathrm{AB}$ & 80.6 & 45,395 & 35,811 & 121,054 & 95,497 & 275 & 931 & 0.4 & 0.5 & 242 & $\begin{array}{l}\text { M. Cambrian } \\
\text { Basal Sands. }\end{array}$ & 3.1 \\
\hline Varman & Sask. & 43 & - & - & - & - & - & - & - & - & 93 & $\begin{array}{l}\text { M. Cambrian } \\
\text { Basal Sands. }\end{array}$ & 1.6 \\
\hline Vegreville & $\mathrm{AB}$ & 64 & 8815 & 6954 & 23,506 & 18,543 & 53 & 181 & 0.2 & 0.1 & 176 & $\begin{array}{l}\text { M. Cambrian } \\
\text { Basal Sands. }\end{array}$ & 2 \\
\hline Vermilion & $\mathrm{AB}$ & 55.8 & - & - & - & - & - & - & - & - & 143 & $\begin{array}{l}\text { M. Cambrian } \\
\text { Basal Sands. }\end{array}$ & 1.8 \\
\hline Virden & Man. & 56 & - & - & - & - & - & - & - & - & 144 & $\begin{array}{l}\text { M. Cambrian } \\
\text { Basal Sands. }\end{array}$ & 1.6 \\
\hline Wainwleft & $\mathrm{AB}$ & 64 & 8815 & 6954 & 23,506 & 18,543 & 53 & 181 & 0.2 & 0.1 & 176 & $\begin{array}{l}\text { M. Cambrian } \\
\text { Basal Sands. }\end{array}$ & 2 \\
\hline Westlock & $\mathrm{AB}$ & 73.6 & 29,970 & 23,643 & 79,919 & 63,047 & 182 & 615 & 0.3 & 0.3 & 214 & $\begin{array}{l}\text { M. Cambrian } \\
\text { Basal Sands. }\end{array}$ & 2.3 \\
\hline Wetaskiwin & $\mathrm{AB}$ & 110 & 110,183 & 86,921 & 293,821 & 231,790 & 669 & 2260 & 0.7 & 1.3 & 359 & $\begin{array}{l}\text { M. Cambrian } \\
\text { Basal Sands. }\end{array}$ & 2.7 \\
\hline Weyburn & Sask. & 92 & 70,076 & 55,282 & 186,870 & 147,418 & 425 & 1437 & 0.5 & 0.8 & 287 & $\begin{array}{l}\text { U. Cambrian } \\
\text { Deadwood }\end{array}$ & 2.7 \\
\hline White City & Sask. & 65 & 9916 & 7823 & 26,444 & 20,861 & 60 & 203 & 0.2 & 0.1 & 178 & $\begin{array}{l}\text { U. Cambrian } \\
\text { Deadwood }\end{array}$ & 2.15 \\
\hline Whitecourt & $\mathrm{AB}$ & 140 & 176,293 & 139,074 & 470,113 & 370,863 & 1070 & 3616 & 1.1 & 2.0 & 479 & $\begin{array}{l}\text { M. Cambrian } \\
\text { Basal Sands. }\end{array}$ & 3.3 \\
\hline Yorkton & Sask. & 46 & - & - & - & - & - & - & - & - & 102 & $\begin{array}{l}\text { M. Cambrian } \\
\text { Basal Sands. }\end{array}$ & 1.3 \\
\hline
\end{tabular}




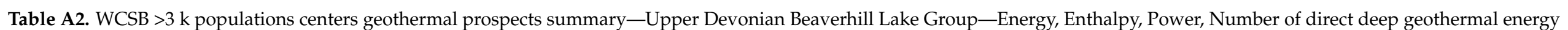
heated households feasible.

\begin{tabular}{|c|c|c|c|c|c|c|c|c|c|c|c|c|c|}
\hline City Location & Province & Temperature $T$ & Energy 1 & Energy 2 & Energy 3 & Energy 4 & Households & Households & Power & Power & $\begin{array}{l}\text { Enthalpy } \\
\text { Gain }\end{array}$ & Formation Group & Depth \\
\hline & & & $\begin{array}{l}\text { at } C=3993 \\
\text { at } 30 \mathrm{~kg} / \mathrm{s}\end{array}$ & $\begin{array}{l}\text { at } C=3150 \\
\text { at } 30 \mathrm{~kg} / \mathrm{s}\end{array}$ & $\begin{array}{l}\text { at } C=3993 \\
\text { at } 80 \mathrm{~kg} / \mathrm{s}\end{array}$ & $\begin{array}{l}\text { at } C=3150 \\
\text { at } 80 \mathrm{~g} / \mathrm{s}\end{array}$ & Minimum & $\begin{array}{l}\text { Maximum } \\
\text { number }\end{array}$ & $\begin{array}{l}\text { at } C=3993 \\
\text { at } 30 \mathrm{~kg} / \mathrm{s}\end{array}$ & $\begin{array}{l}\text { at } C=3150 \\
\text { at } 80 \mathrm{~kg} / \mathrm{s}\end{array}$ & at $\mathrm{C}=3993$ & & \\
\hline Name & Name & ${ }^{\circ} \mathrm{C}$ & GJ Year & GJ Year & GJ Year & GJ Year & $\begin{array}{l}\text { @ 130 } \\
\text { GJ/Year }\end{array}$ & $\begin{array}{l}\text { @ } 130 \\
\text { GJ/Year }\end{array}$ & MW el. & MW el. & $\mathrm{kJ} / \mathrm{kg}$ & & $\mathrm{km}$ \\
\hline Airdrie & $\mathrm{AB}$ & 80 & $44,073.14$ & $34,768.44$ & $117,528.4$ & $92,715.84$ & 267.4495 & 904.0643 & 0.35937 & 0.504 & 239.58 & /Leduc & 1.9 \\
\hline Banff & $\mathrm{AB}$ & $?$ & - & - & - & - & - & - & - & - & & Disturbed belt & $?$ \\
\hline Barrhead & $\mathrm{AB}$ & 59.2 & - & - & - & - & - & - & - & - & 156.5256 & $\begin{array}{l}\text { U. Devonian } \\
\text { Beaverhill L. }\end{array}$ & 1.85 \\
\hline Battleford & Sask. & - & - & - & - & - & - & - & - & - & - & & - \\
\hline Beaumont & $\mathrm{AB}$ & 60 & - & - & - & - & - & - & - & - & 159.72 & $\begin{array}{l}\text { Beaverhill } 1 . \\
\text { Group }\end{array}$ & 1.7 \\
\hline Blackfalds & $\mathrm{AB}$ & 85 & $55,091.42$ & $43,460.55$ & $146,910.5$ & $115,894.8$ & 334.3119 & 1130.08 & - & 0.63 & 259.545 & $\begin{array}{l}\text { U. Devonian } \\
\text { Beaverhill L. }\end{array}$ & 2.5 \\
\hline Bonnyville & $\mathrm{AB}$ & - & - & - & - & - & - & - & - & - & - & Shallow basin & \\
\hline Brooks & $\mathrm{AB}$ & 50 & - & - & - & - & - & - & - & - & 119.79 & $\begin{array}{l}\text { Beaverhill } 1 . \\
\text { Group }\end{array}$ & 1.7 \\
\hline Calgary & $\mathrm{AB}$ & 80 & $44,073.14$ & $34,768.44$ & $117,528.4$ & $92,715.84$ & 267.4495 & 904.0643 & 0.35937 & 0.504 & 239.58 & /Leduc & 3.4 \\
\hline Camrose & $\mathrm{AB}$ & 60 & - & - & - & - & - & - & 0.11979 & - & 159.72 & $\begin{array}{l}\text { Beaverhill } 1 . \\
\text { Group }\end{array}$ & 1.6 \\
\hline Canmore & $\mathrm{AB}$ & - & - & - & - & - & - & - & - & - & - & Disturbed belt & - \\
\hline Cardston & $\mathrm{AB}$ & 66 & $13,221.94$ & $10,430.53$ & $35,258.51$ & $27,814.75$ & 80.23486 & 271.2193 & - & - & 183.678 & $\begin{array}{l}\text { U. Devonian } \\
\text { BeaverbillL }\end{array}$ & 3.3 \\
\hline $\begin{array}{l}\text { Carstairs } \\
\text { Chestermere }\end{array}$ & $\begin{array}{l}\mathrm{AB} \\
\mathrm{AB}\end{array}$ & $\begin{array}{l}75.6 \\
75\end{array}$ & $\begin{array}{l}34,377.05 \\
33,054.85\end{array}$ & $\begin{array}{l}27,119.38 \\
26,076.33\end{array}$ & $\begin{array}{l}91,672.12 \\
88,146.27\end{array}$ & $\begin{array}{l}72,318.36 \\
69,536.88\end{array}$ & $\begin{array}{l}208.6106 \\
200.5872\end{array}$ & $\begin{array}{l}705.1702 \\
678.0483\end{array}$ & - & - & $\begin{array}{l}222.0108 \\
219.615\end{array}$ & $\begin{array}{l}\text { Leduc F. } \\
\text { /Leduc }\end{array}$ & $\begin{array}{l}3.15 \\
3\end{array}$ \\
\hline Claresholm & $\mathrm{AB}$ & 70.35 & $22,807.85$ & $17,992.67$ & $60,820.93$ & $47,980.45$ & 138.4051 & 467.8533 & - & - & 201.0476 & $\begin{array}{l}\text { U. Devonian } \\
\text { Beaverhill L. }\end{array}$ & 3.35 \\
\hline Coaldale & $\mathrm{AB}$ & 47.5 & - & - & - & - & - & - & - & - & 109.8075 & $\begin{array}{l}\text { U. Devonian } \\
\text { Beaverhill L. }\end{array}$ & 1.9 \\
\hline Cochrane & $\mathrm{AB}$ & - & - & - & - & - & - & - & - & - & - & Disturbed belt & - \\
\hline $\begin{array}{l}\text { Cold Lake } \\
\text { Cold Lake }\end{array}$ & $\begin{array}{l}\mathrm{AB} \\
\mathrm{AB}\end{array}$ & - & - & - & - & - & - & - & - & - & - & $\begin{array}{l}\text { Shallow basin } \\
\text { Shallow basin }\end{array}$ & - \\
\hline Dawson Creek & NE BC & $\overline{126}$ & $145,441.4$ & $114,735.9$ & $387,843.6$ & $30,5962.3$ & 882.5835 & 2983.412 & 0.910404 & $\begin{array}{l}- \\
1.6632\end{array}$ & 423.258 & $\begin{array}{l}\text { U. Devonian } \\
\text { Beaverhill L }\end{array}$ & 3.6 \\
\hline $\begin{array}{l}\text { Devon } \\
\text { Didsbury }\end{array}$ & $\begin{array}{l}\mathrm{AB} \\
\mathrm{AB}\end{array}$ & $\begin{array}{l}72.2 \\
78.75\end{array}$ & $\begin{array}{l}26,884.61 \\
41,318.57\end{array}$ & $\begin{array}{l}21,208.75 \\
32,595.41\end{array}$ & $\begin{array}{l}71,692.3 \\
110,182.8\end{array}$ & $\begin{array}{l}56,556.66 \\
86,921.1\end{array}$ & $\begin{array}{l}163.1442 \\
250.7339\end{array}$ & $\begin{array}{l}551.4793 \\
847.5603\end{array}$ & $\begin{array}{l}- \\
-\end{array}$ & $\begin{array}{l}- \\
-\end{array}$ & $\begin{array}{l}208.4346 \\
234.5888\end{array}$ & $\begin{array}{l}\text { Cooking Lk F. } \\
\text { Leduc F. }\end{array}$ & $\begin{array}{l}1.9 \\
3.15\end{array}$ \\
\hline Drayton Valley & $\mathrm{AB}$ & 86.8 & 59,058 & $46,589.71$ & 157,488 & $124,239.2$ & 358.3824 & 1211.446 & _- & 0.67536 & 266.7324 & $\begin{array}{l}\text { U. Devonian } \\
\text { Beaverhill L }\end{array}$ & 2.8 \\
\hline Drumheller & $\mathrm{AB}$ & 51.3 & _- & _- & _- & _- & _- & _- & _- & _- & 124.9809 & Leduc F. & 1.9 \\
\hline E.Lloydminster & Sask. & - & - & - & - & - & - & - & - & - & - & & - \\
\hline Edmonton & $\mathrm{AB}$ & 70 & $22,036.57$ & $17,384.22$ & $58,764.18$ & $46,357.92$ & 133.7248 & 452.0322 & - & - & 199.65 & $\begin{array}{l}\text { Beaverhill } 1 . \\
\text { Group }\end{array}$ & 1.7 \\
\hline Edson & $\mathrm{AB}$ & 126 & $145,441.4$ & $114,735.9$ & $387,843.6$ & $30,5962.3$ & 882.5835 & 2983.412 & 0.910404 & 1.6632 & 423.258 & $\begin{array}{l}\text { Beaverhill } 1 . \\
\text { Group }\end{array}$ & 3.6 \\
\hline Estevan & Sask. & 82.8 & $50,243.38$ & $39,636.02$ & $133,982.3$ & $105,696.1$ & 304.8925 & 1030.633 & 0.392911 & 0.57456 & 250.7604 & $\begin{array}{l}\text { U. Devonian } \\
\text { Beaverhill L. }\end{array}$ & 2.3 \\
\hline Fort Liard & NWT & 136 & $167,477.9$ & $132,120.1$ & $446,607.8$ & $352,320.2$ & 1016.308 & 3435.445 & 1.030194 & 1.9152 & 463.188 & $\begin{array}{l}\text { U. Devonian } \\
\text { Beaverhill L. }\end{array}$ & 3.4 \\
\hline
\end{tabular}


Table A2. Cont.

\begin{tabular}{|c|c|c|c|c|c|c|c|c|c|c|c|c|c|}
\hline City Location & Province & Temperature $\mathrm{T}$ & Energy 1 & Energy 2 & Energy 3 & Energy 4 & Households & Households & Power & Power & $\begin{array}{l}\text { Enthalpy } \\
\text { Gain }\end{array}$ & Formation Group & Depth \\
\hline & & & $\begin{array}{l}\text { at } C=3993 \\
\text { at } 30 \mathrm{~kg} / \mathrm{s}\end{array}$ & $\begin{array}{l}\text { at } C=3150 \\
\text { at } 30 \mathrm{~kg} / \mathrm{s}\end{array}$ & $\begin{array}{l}\text { at } C=3993 \\
\text { at } 80 \mathrm{~kg} / \mathrm{s}\end{array}$ & $\begin{array}{l}\text { at } C=3150 \\
\text { at } 80 \mathrm{~g} / \mathrm{s}\end{array}$ & $\begin{array}{l}\text { Minimum } \\
\text { number }\end{array}$ & $\begin{array}{l}\text { Maximum } \\
\text { number }\end{array}$ & $\begin{array}{l}\text { at } C=3993 \\
\text { at } 30 \mathrm{~kg} / \mathrm{s}\end{array}$ & $\begin{array}{l}\text { at } C=3150 \\
\text { at } 80 \mathrm{~kg} / \mathrm{s}\end{array}$ & at $C=3993$ & & \\
\hline Name & Name & ${ }^{\circ} \mathrm{C}$ & GJ Year & GJ Year & GJ Year & GJ Year & $\begin{array}{l}\text { @ } 130 \\
\text { GJ/Year }\end{array}$ & $\begin{array}{l}@ 130 \\
\text { GJ/Year }\end{array}$ & MW el. & MW el. & $\mathrm{kJ} / \mathrm{kg}$ & & $\mathbf{k m}$ \\
\hline Fort Nelson & NE BC & 84 & $52,887.76$ & $41,722.13$ & 141,034 & 111,259 & 320.9394 & 1084.877 & - & 0.6048 & 255.552 & \multirow{7}{*}{$\begin{array}{l}\text { U. Devonian } \\
\text { Beaverhill L. } \\
\text { Beaverhill l. } \\
\text { Group } \\
\text { U. Devonian } \\
\text { Beaverhill L. } \\
\text { Shallow basin } \\
\text { Disturbed belt } \\
\text { /Leduc } \\
\text { Beaverhill l. } \\
\text { Group } \\
\text { /Leduc }\end{array}$} & 2.1 \\
\hline Fort Sask. & $\mathrm{AB}$ & 60 & - & - & - & - & - & - & - & - & 159.72 & & 1.6 \\
\hline Fort St. John & NE BC & 112 & $114,590.2$ & $90,397.94$ & $305,573.7$ & $241,061.2$ & 695.3688 & 2350.567 & 0.742698 & 1.3104 & 367.356 & & 3.5 \\
\hline $\begin{array}{l}\text { Grand Centre } \\
\text { Grande Cache }\end{array}$ & $\begin{array}{l}\mathrm{AB} \\
\mathrm{AB}\end{array}$ & & - & - & - & - & - & - & & - & & & - \\
\hline Grande Prairie & $\mathrm{AB}$ & 90 & $\overline{6} 6,109.71$ & $\overline{5} 2,152.66$ & $\overline{176,292.5}$ & $\overline{139}, 073.8$ & $\overline{4} 01.1743$ & $\overline{1} 356.097$ & 0.47916 & $\overline{0.756}$ & 279.51 & & $\overline{3} .5$ \\
\hline High River & $\mathrm{AB}$ & 80 & $44,073.14$ & $34,768.44$ & $117,528.4$ & $92,715.84$ & 267.4495 & 904.0643 & 0.35937 & 0.504 & 239.58 & & 3.5 \\
\hline Hinton & $\mathrm{AB}$ & 160 & $220,365.7$ & $173,842.2$ & $587,641.8$ & $463,579.2$ & 1337.248 & 4520.322 & 1.31769 & 2.52 & 559.02 & & $3.4-5.4$ \\
\hline Humboldt & Sask. & - & - & - & - & - & - & - & - & - & - & \multirow{12}{*}{ 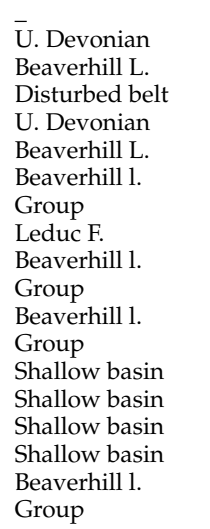 } & - \\
\hline Innisfail & $\mathrm{AB}$ & 68.75 & 19,282 & $15,211.19$ & $51,418.66$ & $40,563.18$ & 117.0092 & 395.5282 & - & - & 194.6588 & & 2.75 \\
\hline Jasper & $\mathrm{AB}$ & - & - & - & - & - & - & - & - & - & - & & \\
\hline Kindersley & Sask. & 32.4 & - & - & - & - & - & - & - & - & 49.5132 & & 1.2 \\
\hline Lacombe & $\mathrm{AB}$ & 130 & 154,256 & $121,689.5$ & $411,349.3$ & $324,505.4$ & 936.0734 & 3164.225 & 0.95832 & 1.764 & 439.23 & & 2.3 \\
\hline Langdon & $\mathrm{AB}$ & 69.6 & $21,155.11$ & $16,688.85$ & $56,413.62$ & $44,503.6$ & 128.3758 & 433.9509 & _ & _- & 198.0528 & & 2.9 \\
\hline Leduc & $\mathrm{AB}$ & 80 & $44,073.14$ & $34,768.44$ & $117,528.4$ & $92,715.84$ & 267.4495 & 904.0643 & - & 0.504 & 239.58 & & 1.9 \\
\hline Lethbridge & $\mathrm{AB}$ & 60 & - & - & - & - & - & - & - & - & 159.72 & & 2.3 \\
\hline $\begin{array}{l}\text { Lloydminster } \\
\text { Malville }\end{array}$ & $\begin{array}{l}\mathrm{AB} \\
\text { Sask }\end{array}$ & - & - & - & - & - & - & - & - & - & - & & - \\
\hline Martensville & $\begin{array}{l}\text { Sask. } \\
\text { Sask. }\end{array}$ & - & - & - & - & - & - & - & - & - & - & & - \\
\hline Meadow Lake & Sask. & - & - & - & - & $\begin{array}{l}- \\
-\end{array}$ & - & $\begin{array}{l}- \\
-\end{array}$ & $\begin{array}{l}- \\
-\end{array}$ & $\begin{array}{l}- \\
-\end{array}$ & $\begin{array}{l}- \\
-\end{array}$ & & $\begin{array}{l}- \\
-\end{array}$ \\
\hline Medicine Hat & $\mathrm{AB}$ & 50 & - & - & - & - & - & - & - & - & 119.79 & & 1.6 \\
\hline Melford & Sask. & - & - & - & - & - & - & - & - & - & - & \multirow{3}{*}{$\begin{array}{l}\text { U. Devonian } \\
\text { Beaverhill L. } \\
\text { /Cooking Lk F. }\end{array}$} & - \\
\hline Moose Jaw & Sask. & 37.5 & - & - & - & - & - & - & - & - & 69.8775 & & 1.5 \\
\hline Morinville & $\mathrm{AB}$ & 70 & $22,036.57$ & $17,384.22$ & $58,764.18$ & $46,357.92$ & 133.7248 & 452.0322 & - & - & 199.65 & & 1.6 \\
\hline $\begin{array}{l}\text { North } \\
\text { Battleford }\end{array}$ & Sask. & - & - & - & - & - & - & - & - & - & - & Shallow basin & - \\
\hline Olds & $\mathrm{AB}$ & 76.25 & $35,809.42$ & $28,249.36$ & $95,491.8$ & $75,331.62$ & 217.3028 & 734.5523 & - & - & 224.6063 & \multirow{5}{*}{$\begin{array}{l}\text { Leduc F. } \\
\text { Leduc F. } \\
\text { U. Devonian } \\
\text { Beaverhill L. } \\
\text { Disturbed belt } \\
\text { U. Devonian } \\
\text { Beaverhill L. }\end{array}$} & 3.05 \\
\hline Peace River & $A B$ & 67.2 & $15,866.33$ & $12,516.64$ & $42,310.21$ & $33,377.7$ & 96.28183 & 325.4632 & - & - & 188.4696 & & 2.1 \\
\hline Penhold & $\mathrm{AB}$ & 70.2 & $22,477.3$ & $17,731.9$ & $59,939.47$ & $47,285.08$ & 136.3993 & 461.0728 & _ & _- & 200.4486 & & 2.6 \\
\hline Pincher Creek & $\mathrm{AB}$ & - & - & - & - & - & - & - & - & - & & & - \\
\hline Ponoka & $\mathrm{AB}$ & 88 & $61,702.39$ & $48,675.82$ & $164,539.7$ & $129,802.2$ & 374.4294 & 1265.69 & - & 0.7056 & 271.524 & & 2.2 \\
\hline
\end{tabular}


Table A2. Cont.

\begin{tabular}{|c|c|c|c|c|c|c|c|c|c|c|c|c|c|}
\hline City Location & Province & Temperature $\mathrm{T}$ & Energy 1 & Energy 2 & Energy 3 & Energy 4 & Households & Households & Power & Power & $\begin{array}{l}\text { Enthalpy } \\
\text { Gain }\end{array}$ & Formation Group & Depth \\
\hline & & & $\begin{array}{l}\text { at } C=3993 \\
\text { at } 30 \mathrm{~kg} / \mathrm{s}\end{array}$ & $\begin{array}{l}\text { at } C=3150 \\
\text { at } 30 \mathrm{~kg} / \mathrm{s}\end{array}$ & $\begin{array}{l}\text { at } C=3993 \\
\text { at } 80 \mathrm{~kg} / \mathrm{s}\end{array}$ & $\begin{array}{l}\text { at } C=3150 \\
\text { at } 80 \mathrm{~g} / \mathrm{s}\end{array}$ & $\begin{array}{l}\text { Minimum } \\
\text { number }\end{array}$ & $\begin{array}{l}\text { Maximum } \\
\text { number }\end{array}$ & $\begin{array}{l}\text { at } C=3993 \\
\text { at } 30 \mathrm{~kg} / \mathrm{s}\end{array}$ & $\begin{array}{l}\text { at } C=3150 \\
\text { at } 80 \mathrm{~kg} / \mathrm{s}\end{array}$ & at $C=3993$ & & \\
\hline Name & Name & ${ }^{\circ} \mathrm{C}$ & GJ Year & GJ Year & GJ Year & GJ Year & $\begin{array}{l}\text { @ } 130 \\
\text { GJ/Year }\end{array}$ & $\begin{array}{l}\text { @ 130 } \\
\text { GJ/Year }\end{array}$ & MW el. & MW el. & $\mathrm{kJ} / \mathrm{kg}$ & & $\mathrm{km}$ \\
\hline Prince Albert & Sask. & - & - & - & - & - & - & - & - & - & - & Shallow basin & - \\
\hline Raymond & $\mathrm{AB}$ & & & & & & & & _- & & 111.804 & $\begin{array}{l}\text { U. Devonian } \\
\text { Beaverhill L. }\end{array}$ & 2 \\
\hline Red Deer & $\mathrm{AB}$ & 90 & $66,109.71$ & $52,152.66$ & $176,292.5$ & $139,073.8$ & 401.1743 & 1356.097 & - & 0.756 & 279.51 & /Leduc F. to SE & 2.6 \\
\hline Redcliff & $\mathrm{AB}$ & 45.375 & - & - & - & - & - & - & - & - & 101.3224 & $\begin{array}{l}\text { U. Devonian } \\
\text { Beaverhill L. }\end{array}$ & 1.65 \\
\hline Regina & Sask. & 42 & - & - & - & - & - & - & - & - & 87.846 & $\begin{array}{l}\text { U. Devonian } \\
\text { Beaverhill L. }\end{array}$ & 1.4 \\
\hline $\begin{array}{l}\text { Rocky } \\
\text { Mountain } \\
\text { House }\end{array}$ & $\mathrm{AB}$ & 108.5 & $106,877.4$ & $84,313.47$ & $285,006.3$ & $224,835.9$ & 648.5651 & 2192.356 & 0.700772 & 1.2222 & 353.3805 & $\begin{array}{l}\text { U. Devonian } \\
\text { Beaverhill L. }\end{array}$ & 3.5 \\
\hline Saskatoon & Sask. & _- & _- & _- & - & _- & _- & - & _- & _- & _- & Shallow basin & _- \\
\hline Slave Lake & $\mathrm{AB}$ & 54.4 & - & - & - & - & - & - & - & - & 137.3592 & $\begin{array}{l}\text { U. Devonian } \\
\text { Beaverhill L. }\end{array}$ & 1.6 \\
\hline Spruce Grove & $\mathrm{AB}$ & 70 & $22,036.57$ & $17,384.22$ & $58,764.18$ & $46,357.92$ & 133.7248 & 452.0322 & _- & _- & 199.65 & Beaverhill l. & 1.9 \\
\hline St. Albert & $\mathrm{AB}$ & 60 & - & - & - & - & - & - & _- & _- & 159.72 & /Cooking Lk F. & 1.7 \\
\hline St. Paul & $\mathrm{AB}$ & & - & - & - & - & - & - & - & - & -79.86 & Shallow basin & \\
\hline Stettler & $\mathrm{AB}$ & 55.5 & - & - & - & - & - & - & - & - & 141.7515 & Leduc F. & $\overline{1} .85$ \\
\hline Stony Plain & $\mathrm{AB}$ & 70 & $22,036.57$ & $17,384.22$ & $58,764.18$ & $46,357.92$ & 133.7248 & 452.0322 & - & - & 199.65 & $\begin{array}{l}\text { Beaverhill } 1 . \\
\text { Group }\end{array}$ & 2 \\
\hline Swift Current & Sask. & 45.9 & - & - & - & - & - & - & - & - & 103.4187 & $\begin{array}{l}\text { U. Devonian } \\
\text { Beaverhill L. }\end{array}$ & 1.7 \\
\hline Taber & $\mathrm{AB}$ & 45.9 & - & - & - & - & - & - & - & - & 103.4187 & $\begin{array}{l}\text { U. Devonian } \\
\text { Beaverhill L. }\end{array}$ & 1.7 \\
\hline Three Hills & $\mathrm{AB}$ & 59.8 & - & - & - & - & - & - & - & - & 158.9214 & $\begin{array}{l}\text { U. Devonian } \\
\text { Beaverhill L. }\end{array}$ & 2.3 \\
\hline Varman & Sask. & - & - & - & - & - & - & - & - & - & - & Shallow basin & - \\
\hline Vegreville & $\mathrm{AB}$ & 35.2 & - & - & - & - & - & - & - & - & 60.6936 & $\begin{array}{l}\text { U. Devonian } \\
\text { Beaverhill L. }\end{array}$ & 1.1 \\
\hline $\begin{array}{l}\text { Vermilion } \\
\text { Virden Man. }\end{array}$ & $\begin{array}{l}\mathrm{AB} \\
\text { Man. }\end{array}$ & & - & - & - & - & - & - & - & - & - & Shallow basin & - \\
\hline $\begin{array}{l}\text { Virden Man. } \\
\text { Wainwright }\end{array}$ & $\begin{array}{l}\text { Man. } \\
\text { AB }\end{array}$ & $\begin{array}{l}- \\
33.6\end{array}$ & - & - & - & - & - & - & - & - & $\begin{array}{l}- \\
54.3048\end{array}$ & $\begin{array}{l}\text { Shallow basin } \\
\text { U. Devonian }\end{array}$ & $\begin{array}{l}- \\
1.05\end{array}$ \\
\hline Westlock & $\mathrm{AB}$ & 51.2 & - & - & - & - & - & - & - & - & 124.5816 & $\begin{array}{l}\text { Beaverhill L. } \\
\text { U. Devonian } \\
\text { Beaverhill L. }\end{array}$ & 1.6 \\
\hline Wetaskiwin & $\mathrm{AB}$ & 80 & $44,073.14$ & $34,768.44$ & $117,528.4$ & $92,715.84$ & 267.4495 & 904.0643 & - & - & 239.58 & $\begin{array}{l}\text { Beaverhill } 1 . \\
\text { Group }\end{array}$ & 2 \\
\hline Weyburn & Sask. & 66.3 & $13,883.04$ & $10,952.06$ & $37,021.43$ & $29,205.49$ & 84.2466 & 284.7803 & - & - & 184.8759 & $\begin{array}{l}\text { U. Devonian } \\
\text { Beaverhill L. }\end{array}$ & 1.95 \\
\hline White City & Sask. & 42 & - & - & - & _- & - & - & - & - & 87.846 & $\begin{array}{l}\text { U. Devonian } \\
\text { Beaverhill L }\end{array}$ & 1.4 \\
\hline Whitecourt & $\mathrm{AB}$ & 110 & $110,182.8$ & $86,921.1$ & $293,820.9$ & $231,789.6$ & 668.6238 & 2260.161 & 0.71874 & 1.26 & 359.37 & Swan H. - Slave Pt & 2.7 \\
\hline Yorkton & Sask. & - & - & - & - & - & - & _- & _- & - & - & Shallow basin & - \\
\hline
\end{tabular}




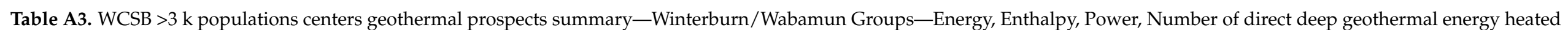
households feasible.

\begin{tabular}{|c|c|c|c|c|c|c|c|c|c|c|c|c|c|}
\hline City Location & Province & Temperature $\mathbf{T}$ & Energy 1 & Energy 2 & Energy 3 & Energy 4 & Households & Households & Power & Power & $\begin{array}{l}\text { Enthalpy } \\
\text { Gain }\end{array}$ & Formation Group & Depth \\
\hline \multirow[b]{3}{*}{ Name } & \multirow[b]{3}{*}{ Name } & \multirow[b]{3}{*}{${ }^{\circ} \mathrm{C}$} & \multirow{3}{*}{$\begin{array}{l}\text { at } C=3993 \\
\text { at } 30 \mathrm{~kg} / \mathrm{s} \\
\text { GJ Year }\end{array}$} & \multirow{3}{*}{$\begin{array}{l}\text { at } C=3150 \\
\text { at } 30 \mathrm{~kg} / \mathrm{s} \\
\text { GJ Year }\end{array}$} & \multirow{3}{*}{$\begin{array}{l}\text { at } C=3993 \\
\text { at } 80 \mathrm{~kg} / \mathrm{s} \\
\text { GJ Year }\end{array}$} & \multirow{3}{*}{$\begin{array}{l}\text { at } C=3150 \\
\text { at } 80 \mathrm{~kg} / \mathrm{s} \\
\text { GJ Year }\end{array}$} & \multirow{3}{*}{$\begin{array}{l}\text { Minimum } \\
\text { number } \\
@ 130 \\
\text { GJ/Year }\end{array}$} & \multirow{3}{*}{$\begin{array}{l}\text { Maximum } \\
\text { number } \\
@ 130 \\
\text { GJ/Year }\end{array}$} & \multirow{3}{*}{$\begin{array}{l}\text { at } C=3993 \\
\text { at } 30 \mathrm{~kg} / \mathrm{s} \\
\text { MW el. }\end{array}$} & \multirow{3}{*}{$\begin{array}{l}\text { at } C=3150 \\
\text { at } 80 \mathrm{~kg} / \mathrm{s} \\
\mathrm{MW} \mathrm{el.}\end{array}$} & \multirow{3}{*}{$\begin{array}{l}\text { at } C=3993 \\
\mathrm{~kJ} / \mathrm{kg}\end{array}$} & \multirow[t]{3}{*}{$\begin{array}{l}\text { top of } \\
\text { Winterburn } \\
\text { Group }\end{array}$} & \multirow[b]{3}{*}{$\mathrm{km}$} \\
\hline & & & & & & & & & & & & & \\
\hline & & & & & & & & & & & & & \\
\hline Airdrie & $\mathrm{AB}$ & 70 & $22,036.57$ & $17,384.22$ & $58,764.18$ & $46,357.92$ & 133.7248 & 452.0322 & 0.23958 & 0.252 & 199.65 & /Dolomite Nisku & 2.9 \\
\hline Banff & $\mathrm{AB}$ & - & - & - & - & - & - & - & - & - & - & Disturbed belt & - \\
\hline Barrhead & $\mathrm{AB}$ & 44.8 & - & - & - & - & - & - & - & - & 99.0264 & $\begin{array}{l}\text { Wabamum } \\
\text { dolomite }\end{array}$ & 1.4 \\
\hline Battleford & Sask. & - & - & - & - & - & - & - & - & - & - & Shallow basin & - \\
\hline Beaumont & $\mathrm{AB}$ & 50 & - & - & - & - & - & - & - & - & 119.79 & $\begin{array}{l}\text { Wabamum } \\
\text { dolomite }\end{array}$ & 1.3 \\
\hline Blackfalds & $\mathrm{AB}$ & 73.1 & $28,867.9$ & $22,773.33$ & $76,981.08$ & $60,728.88$ & 175.1794 & 592.1621 & 0.276715 & 0.33012 & 212.0283 & Dolomite Nisku & 2.15 \\
\hline Bonnyville & $\mathrm{AB}$ & - & - & - & - & - & - & - & - & - & - & Shallow basin & - \\
\hline Brooks & $\mathrm{AB}$ & 40 & - & - & - & - & - & - & - & - & 79.86 & $\begin{array}{l}\text { Winterburn/ } \\
\text { Wabamun }\end{array}$ & 1.4 \\
\hline Calgary & $\mathrm{AB}$ & 70 & $22,036.57$ & $17,384.22$ & $58,764.18$ & $46,357.92$ & 133.7248 & 452.0322 & 0.23958 & 0.252 & 199.65 & /Dolomite Nisku & 3 \\
\hline Camrose & $\mathrm{AB}$ & 50 & - & - & - & - & - & - & - & - & 119.79 & /Dolomite Nisku & 1.3 \\
\hline Canmore & $\mathrm{AB}$ & - & - & - & - & - & - & - & - & $\begin{array}{l}- \\
-\end{array}$ & - & Shallow basin & - \\
\hline Cardston & $\mathrm{AB}$ & 60 & - & - & - & - & - & - & - & - & 159.72 & $\begin{array}{l}\text { top of Winterburn } \\
\text { Group }\end{array}$ & 3 \\
\hline Carstairs & $\mathrm{AB}$ & 68.4 & $18,510.72$ & $14,602.74$ & $49,361.91$ & $38,940.65$ & 112.3288 & 379.707 & 0.220414 & 0.21168 & 193.2612 & Dolimite Nisku & 2.85 \\
\hline Chestermere & $\mathrm{AB}$ & 70 & $22,036.57$ & $17,384.22$ & $58,764.18$ & $46,357.92$ & 133.7248 & 452.0322 & 0.23958 & 0.252 & 199.65 & /Dolomite Nisku & 2.8 \\
\hline Claresholm & $\mathrm{AB}$ & 60.9 & 1983.291 & 1564.58 & 5288.776 & 4172.213 & 12.03523 & 40.6829 & 0.130571 & 0.02268 & 163.3137 & $\begin{array}{l}\text { top of Winterburn } \\
\text { Group }\end{array}$ & 2.9 \\
\hline Coaldale & $\mathrm{AB}$ & - & - & - & - & - & - & - & - & - & 79.86 & $\begin{array}{l}\text { top of Winterburn } \\
\text { Group }\end{array}$ & 1.6 \\
\hline Cochrane & $\begin{array}{l}A B \\
A B\end{array}$ & - & - & - & - & - & - & - & - & - & - & Shallow basin & - \\
\hline $\begin{array}{l}\text { Cold Lake } \\
\text { Cold Lake }\end{array}$ & $\mathrm{AB}$ & - & - & _- & _- & _- & - & _- & - & - & - & $\begin{array}{l}\text { Shallow basin } \\
\text { Shallow basin }\end{array}$ & - \\
\hline Dawson Creek & NE BC & 131.25 & $157,010.5$ & $123,862.6$ & $418,694.8$ & $330,300.2$ & 952.789 & 3220.729 & 0.973294 & 1.7955 & 444.2213 & $\begin{array}{l}\text { top of Winterburn } \\
\text { Group }\end{array}$ & 3.75 \\
\hline Devon & $\mathrm{AB}$ & 57 & & & & & & & & & 147.741 & $\begin{array}{l}\text { Wabamum } \\
\text { dolomite }\end{array}$ & 1.5 \\
\hline Didsbury & $\mathrm{AB}$ & 70 & $22,036.57$ & $17,384.22$ & $58,764.18$ & $46,357.92$ & 133.7248 & 452.0322 & 0.23958 & 0.252 & 199.65 & Dolomite Nisku & 2.8 \\
\hline Drayton Valley & $\mathrm{AB}$ & 72 & $26,443.88$ & $20,861.06$ & $70,517.02$ & $55,629.5$ & 160.4697 & 542.4386 & 0.263538 & 0.3024 & 207.636 & $\begin{array}{l}\text { Wabamum } \\
\text { dolomite }\end{array}$ & 2.4 \\
\hline Drumheller & $\mathrm{AB}$ & 45.9 & - & - & - & - & - & - & - & - & 103.4187 & Dolomite Nisku & 1.7 \\
\hline E.Lloydminster & Sask. & 0 & - & - & - & - & - & - & - & - & & Shallow basin & \\
\hline Edmonton & $\mathrm{AB}$ & 50 & - & - & - & - & - & - & - & - & 119.79 & /Dolomite Nisku & 1.2 \\
\hline Edson & $\mathrm{AB}$ & 112 & $114,590.2$ & $90,397.94$ & $305,573.7$ & $241,061.2$ & 695.3688 & 2350.567 & 0.742698 & 1.3104 & 367.356 & $\begin{array}{l}\text { top of Winterburn } \\
\text { Group }\end{array}$ & 3.2 \\
\hline Estevan & Sask. & 77.4 & $38,343.63$ & $30,248.54$ & $102,249.7$ & $80,662.78$ & 232.6811 & 786.536 & 0.328225 & 0.43848 & 229.1982 & $\begin{array}{l}\text { top of Winterburn } \\
\text { Group }\end{array}$ & 2.15 \\
\hline Fort Liard & NWT & 132 & $158,663.3$ & $125,166.4$ & $423,102.1$ & 333,777 & 962.8183 & 3254.632 & 0.982278 & 1.8144 & 447.216 & $\begin{array}{l}\text { top of Winterburn } \\
\text { Group }\end{array}$ & 3.3 \\
\hline
\end{tabular}


Table A3. Cont.

\begin{tabular}{|c|c|c|c|c|c|c|c|c|c|c|c|c|c|}
\hline City Location & Province & Temperature $\mathrm{T}$ & Energy 1 & Energy 2 & Energy 3 & Energy 4 & Households & Households & Power & Power & $\begin{array}{l}\text { Enthalpy } \\
\text { Gain }\end{array}$ & Formation Group & Depth \\
\hline Name & Name & ${ }^{\circ} \mathrm{C}$ & $\begin{array}{l}\text { at } C=3993 \\
\text { at } 30 \mathrm{~kg} / \mathrm{s} \\
\text { GJ Year }\end{array}$ & $\begin{array}{l}\text { at } C=3150 \\
\text { at } 30 \mathrm{~kg} / \mathrm{s} \\
\text { GJ Year }\end{array}$ & $\begin{array}{l}\text { at } \mathrm{C}=3993 \\
\text { at } 80 \mathrm{~kg} / \mathrm{s} \\
\text { GJ Year }\end{array}$ & $\begin{array}{l}\text { at } C=3150 \\
\text { at } 80 \mathrm{~kg} / \mathrm{s} \\
\text { GJ Year }\end{array}$ & $\begin{array}{l}\text { Minimum } \\
\text { number } \\
@ 130 \\
\text { GJ/Year } \\
\end{array}$ & $\begin{array}{l}\text { Maximum } \\
\text { number } \\
@ 130 \\
\text { GJ/Year } \\
\end{array}$ & $\begin{array}{l}\text { at } C=3993 \\
\text { at } 30 \mathrm{~kg} / \mathrm{s} \\
\text { MW el. }\end{array}$ & $\begin{array}{l}\text { at } C=3150 \\
\text { at } 80 \mathrm{~kg} / \mathrm{s} \\
\mathrm{MW} \mathrm{el.}\end{array}$ & at $C=3993$ & $\begin{array}{l}\text { top of } \\
\text { Winterburn } \\
\text { Group }\end{array}$ & km \\
\hline Fort Nelson & NE BC & 56 & - & - & - & - & - & - & 0.071874 & - & 143.748 & $\begin{array}{l}\text { top of Winterburn } \\
\text { Group }\end{array}$ & 1.4 \\
\hline Fort Sask. & $\mathrm{AB}$ & 40 & - & - & - & - & - & - & - & - & 79.86 & /Dolomite Nisku & 1.1 \\
\hline Fort St. John & NE BC & 112 & $114,590.2$ & $90,397.94$ & $305,573.7$ & $241,061.2$ & 695.3688 & 2350.567 & 0.742698 & 1.3104 & 367.356 & $\begin{array}{l}\text { top of Winterburn } \\
\text { Group }\end{array}$ & 3.5 \\
\hline $\begin{array}{l}\text { Grand Centre } \\
\text { Grande Cache }\end{array}$ & $\begin{array}{l}\mathrm{AB} \\
\mathrm{AB}\end{array}$ & - & - & - & - & - & - & - & - & - & - & $\begin{array}{l}\text { Shallow basin } \\
\text { Disturbed belt }\end{array}$ & - \\
\hline Grande Prairie & $\mathrm{AB}$ & $\overline{1} 30$ & $\overline{154,256}$ & $\overline{121,689.5}$ & $\overline{4} 11,349.3$ & $\overline{3} 24,505.4$ & $\overline{9} 36.0734$ & $\overline{3} 164.225$ & $\overline{0} .95832$ & $\overline{1} .764$ & $\overline{4} 39.23$ & /Limestone Nisku & $\overline{3} .4$ \\
\hline High River & $\mathrm{AB}$ & 80 & $44,073.14$ & $34,768.44$ & $117,528.4$ & $92,715.84$ & 267.4495 & 904.0643 & 0.35937 & 0.504 & 239.58 & $\begin{array}{l}\text { Winterburn/ } \\
\text { Wabamun }\end{array}$ & 3.3 \\
\hline Hinton & $\mathrm{AB}$ & 150 & $198,329.1$ & 156,458 & $528,877.6$ & $417,221.3$ & 1203.523 & 4068.29 & 1.1979 & 2.268 & 519.09 & /Dolomite Nisku & $3.7-5.2$ \\
\hline Humboldt & Sask. & 0 & - & - & - & - & - & - & - & - & - & Shallow basin & - \\
\hline Innisfail & $\mathrm{AB}$ & - & - & - & - & - & - & - & - & - & - & Shallow basin & - \\
\hline Jasper & $\mathrm{AB}$ & - & & & & & & & & & & Disturbed belt & - \\
\hline Kindersley & Sask. & & & & & & & & & & & Shallow basin & \\
\hline Lacombe & $\mathrm{AB}$ & $\overline{8} 0$ & $\overline{4} 4,073.14$ & $\overline{3} 4,768.44$ & $\overline{1} 17,528.4$ & $\overline{9} 2,715.84$ & $\overline{2} 67.4495$ & $\overline{9} 04.0643$ & $\overline{0} .35937$ & $\overline{0} .504$ & $\overline{2} 39.58$ & /Dolomite Nisku & $\overline{2}$ \\
\hline Langdon & $\mathrm{AB}$ & 62.4 & 5288.776 & 4172.213 & $14,103.4$ & $11,125.9$ & 32.09394 & 108.4877 & 0.14854 & 0.06048 & 169.3032 & Dolomite Nisku & 2.6 \\
\hline Leduc & $\mathrm{AB}$ & 60 & - & - & - & - & - & - & 0.11979 & - & 159.72 & $\begin{array}{l}\text { Wabamum } \\
\text { dolomite }\end{array}$ & 1.5 \\
\hline Lethbridge & $\mathrm{AB}$ & 50 & - & - & - & _- & - & - & - & - & 119.79 & $\begin{array}{l}\text { Winterburn/ } \\
\text { Wabamun }\end{array}$ & 1.9 \\
\hline Lloydminster & $\mathrm{AB}$ & - & - & - & - & - & - & _- & _- & - & _- & Shallow basin & - \\
\hline Martensville & Sask. & - & - & - & - & - & - & - & - & - & - & Shallow basin & - \\
\hline Meadow Lake & Sask. & - & - & - & - & - & - & $\begin{array}{l}- \\
-\end{array}$ & $\begin{array}{l}- \\
-\end{array}$ & $\begin{array}{l}- \\
-\end{array}$ & $\begin{array}{l}- \\
-\end{array}$ & Shallow basin & - \\
\hline Medicine Hat & Sask. & 40 & - & - & - & - & - & - & - & - & - & $\begin{array}{l}\text { Winterburn/ } \\
\text { Wabamun }\end{array}$ & 1.3 \\
\hline Melford & $\mathrm{AB}$ & - & - & - & - & - & - & - & - & - & - & Shallow basin & - \\
\hline Melville & Sask. & - & - & - & - & - & - & - & - & - & - & Shallow basin & - \\
\hline Moose Jaw & Sask. & 32.5 & - & - & - & - & - & - & - & - & 49.9125 & $\begin{array}{l}\text { top of Winterburn } \\
\text { Group }\end{array}$ & 1.3 \\
\hline Morinville & $\mathrm{AB}$ & 50 & - & - & - & - & - & - & - & - & 119.79 & $\begin{array}{l}\text { Wabamum } \\
\text { dolomite }\end{array}$ & 1.2 \\
\hline $\begin{array}{l}\text { North } \\
\text { Battleford }\end{array}$ & Sask. & - & - & - & - & - & - & - & - & - & - & Shallow basin & - \\
\hline Olds & $\mathrm{AB}$ & 67.5 & $16,527.43$ & $13,038.17$ & $44,073.14$ & $34,768.44$ & 100.2936 & 339.0241 & 0.209633 & 0.189 & 189.6675 & Dolomite Nisku & 2.7 \\
\hline Peace River & $\mathrm{AB}$ & 56 & & & & & & & & & 143.748 & $\begin{array}{l}\text { top of Winterburn } \\
\text { Group }\end{array}$ & 1.75 \\
\hline Penhold & $\mathrm{AB}$ & 62.1 & 4627.679 & 3650.686 & $12,340.48$ & 9735.163 & 28.0822 & 94.92676 & 0.144946 & 0.05292 & 168.1053 & $\begin{array}{l}\text { top of Winterburn } \\
\text { Group }\end{array}$ & 2.3 \\
\hline Pincher Creek & $\mathrm{AB}$ & - & - & - & - & - & - & - & - & - & - & Disturbed belt & - \\
\hline Ponoka & $\mathrm{AB}$ & 72 & $26,443.88$ & $20,861.06$ & $70,517.02$ & $55,629.5$ & 160.4697 & 542.4386 & 0.263538 & 0.3024 & 207.636 & $\begin{array}{l}\text { Winterburn/ } \\
\text { Dolomite Nisku }\end{array}$ & 1.8 \\
\hline
\end{tabular}


Table A3. Cont.

\begin{tabular}{|c|c|c|c|c|c|c|c|c|c|c|c|c|c|}
\hline City Location & Province & Temperature T & Energy 1 & Energy 2 & Energy 3 & Energy 4 & Households & Households & Power & Power & $\begin{array}{l}\text { Enthalpy } \\
\text { Gain }\end{array}$ & Formation Group & Depth \\
\hline Name & Name & ${ }^{\circ} \mathrm{C}$ & $\begin{array}{l}\text { at } C=3993 \\
\text { at } 30 \mathrm{~kg} / \mathrm{s} \\
\text { GJ Year }\end{array}$ & $\begin{array}{l}\text { at } C=3150 \\
\text { at } 30 \mathrm{~kg} / \mathrm{s} \\
\text { GJ Year }\end{array}$ & $\begin{array}{l}\text { at } C=3993 \\
\text { at } 80 \mathrm{~kg} / \mathrm{s} \\
\text { GJ Year }\end{array}$ & $\begin{array}{l}\text { at } C=3150 \\
\text { at } 80 \mathrm{~kg} / \mathrm{s} \\
\text { GJ Year }\end{array}$ & $\begin{array}{l}\text { Minimum } \\
\text { number } \\
@ 130 \\
\text { GJ/Year } \\
\end{array}$ & $\begin{array}{l}\text { Maximum } \\
\text { number } \\
@ 130 \\
\text { GJ/Year } \\
\end{array}$ & $\begin{array}{l}\text { at } C=3993 \\
\text { at } 30 \mathrm{~kg} / \mathrm{s} \\
\text { MW el. }\end{array}$ & $\begin{array}{l}\text { at } C=3150 \\
\text { at } 80 \mathrm{~kg} / \mathrm{s} \\
\mathrm{MW} \mathrm{el.}\end{array}$ & at $C=3993$ & $\begin{array}{l}\text { top of } \\
\text { Winterburn } \\
\text { Group }\end{array}$ & km \\
\hline Prince Albert & Sask. & - & - & - & - & - & - & - & - & - & - & Shallow basin & - \\
\hline Raymond & $\mathrm{AB}$ & 42 & - & - & - & - & - & - & - & - & - & $\begin{array}{l}\text { top of Winterburn } \\
\text { Group }\end{array}$ & 1.75 \\
\hline Red Deer & $\mathrm{AB}$ & 70 & $22,036.57$ & $17,384.22$ & $58,764.18$ & $46,357.92$ & 133.7248 & 452.0322 & 0.23958 & 0.252 & 199.65 & /Dolomite Nisku & 2.2 \\
\hline Redcliff & $\mathrm{AB}$ & 34.375 & & & & & & & & & 57.39938 & $\begin{array}{l}\text { top of Winterburn } \\
\text { Group }\end{array}$ & 1.25 \\
\hline Regina & Sask. & 39 & - & - & - & - & - & - & - & - & 75.867 & $\begin{array}{l}\text { top of Winterburn } \\
\text { Group }\end{array}$ & 1.3 \\
\hline $\begin{array}{l}\text { Rocky } \\
\text { Mountain } \\
\text { House }\end{array}$ & $\mathrm{AB}$ & 93 & $72,720.68$ & $57,367.93$ & $193,921.8$ & $152,981.1$ & 441.2917 & 1491.706 & 0.515097 & 0.8316 & 291.489 & $\begin{array}{l}\text { Wabamum } \\
\text { dolomite }\end{array}$ & 3.1 \\
\hline $\begin{array}{l}\text { Saskatoon } \\
\text { Slave Lake }\end{array}$ & $\begin{array}{l}\text { Sask. } \\
\text { AB }\end{array}$ & $\begin{array}{l}0 \\
37.4\end{array}$ & - & - & - & - & - & - & - & - & $\overline{6} 9.4782$ & $\begin{array}{l}\text { Shallow basin } \\
\text { Dolomite Nisku }\end{array}$ & $\overline{1} .1$ \\
\hline Spruce Grove & $\mathrm{AB}$ & 60 & - & - & $\begin{array}{l}- \\
-\end{array}$ & $\begin{array}{l}- \\
-\end{array}$ & - & $\begin{array}{l}- \\
-\end{array}$ & $\begin{array}{l}- \\
0.11979\end{array}$ & - & 159.72 & $\begin{array}{l}\text { Wabamum } \\
\text { dolomite }\end{array}$ & 1.6 \\
\hline St. Albert & $\mathrm{AB}$ & 50 & - & - & - & - & - & - & - & - & 119.79 & $\begin{array}{l}\text { Wabamum } \\
\text { dolomite }\end{array}$ & 1.3 \\
\hline St. Paul & $\mathrm{AB}$ & - & - & - & - & - & - & - & - & - & - & Shallow basin & - \\
\hline Stettler & $\mathrm{AB}$ & 43.5 & - & & & & & & & & 93.8355 & $\begin{array}{l}\text { top of Winterburn } \\
\text { Group }\end{array}$ & 1.5 \\
\hline Stony Plain & $\mathrm{AB}$ & 60 & - & - & - & - & - & - & 0.11979 & - & 159.72 & $\begin{array}{l}\text { Wabamum } \\
\text { dolomite }\end{array}$ & 1.5 \\
\hline Swift Current & Sask. & 40.5 & - & - & - & - & - & - & - & - & 81.8565 & $\begin{array}{l}\text { top of Winterburn } \\
\text { Group }\end{array}$ & 1.5 \\
\hline Taber & $\mathrm{AB}$ & 37.8 & - & - & - & - & - & - & - & - & 71.0754 & $\begin{array}{l}\text { top of Winterburn } \\
\text { Group }\end{array}$ & 1.4 \\
\hline Three Hills & $\mathrm{AB}$ & 50.7 & - & - & - & - & - & - & - & - & 122.5851 & Dolimite Nisku & 1.95 \\
\hline Vegreville & $\mathrm{AB}$ & & - & - & - & - & - & - & - & - & - & Shallow basin & - \\
\hline Vermilion & $\mathrm{AB}$ & $\overline{0}$ & - & - & - & - & - & - & - & - & - & Shallow basin & - \\
\hline Virden Man. & Man. & 0 & $\begin{array}{l}- \\
-\end{array}$ & - & - & - & $\begin{array}{l}- \\
-\end{array}$ & - & - & $\begin{array}{lll}- \\
-\end{array}$ & $\begin{array}{l}- \\
-\end{array}$ & Shallow basin & - \\
\hline Wainwright & $\mathrm{AB}$ & 0 & - & - & - & - & - & - & - & - & - & Shallow basin & $\begin{array}{l}- \\
-\end{array}$ \\
\hline Warman & Sask. & 0 & - & - & - & - & - & - & - & - & - & Shallow basin & - \\
\hline Westlock & $\mathrm{AB}$ & 38.4 & _- & _- & _- & - & - & - & - & - & 73.4712 & $\begin{array}{l}\text { Wabamum } \\
\text { dolomite }\end{array}$ & 1.2 \\
\hline Wetaskiwin & $\mathrm{AB}$ & 60 & - & - & - & - & - & - & 0.11979 & - & 159.72 & /Dolomite Nisku & 1.6 \\
\hline Weyburn & Sask. & 57.8 & - & - & - & - & - & - & 0.093436 & - & 150.9354 & $\begin{array}{l}\text { top of Winterburn } \\
\text { Group }\end{array}$ & 1.7 \\
\hline White City & Sask. & 39 & _- & _- & _- & _- & - & _- & _- & _- & 75.867 & $\begin{array}{l}\text { top of Winterburn } \\
\text { Group }\end{array}$ & 1.3 \\
\hline Whitecourt & $\mathrm{AB}$ & 90 & $66,109.71$ & $52,152.66$ & $176,292.5$ & $139,073.8$ & 401.1743 & 1356.097 & 0.47916 & 0.756 & 279.51 & Winterburn/Wabamur & 12.3 \\
\hline Yorkton & Sask. & _- & - & - & - & - & - & - & - & - & - & Shallow basin & - \\
\hline
\end{tabular}





feasible.

\begin{tabular}{|c|c|c|c|c|c|c|c|c|c|c|c|c|c|}
\hline City Location & Province & Temperature $T$ & Energy 1 & Energy 2 & Energy 3 & Energy 4 & Households & Households & Power & Power & $\begin{array}{l}\text { Enthalpy } \\
\text { Gain }\end{array}$ & Formation Group & Depth \\
\hline & & & at $C=3993$ & at $C=3150$ & at $C=3993$ & at $C=3150$ & Minimum & Maximum & at $C=3993$ & at $C=3150$ & at $C=3993$ & $\begin{array}{l}\text { top of } \\
\text { Winterburn } \\
\text { Group }\end{array}$ & \\
\hline & & & at $30 \mathrm{~kg} / \mathrm{s}$ & at $30 \mathrm{~kg} / \mathrm{s}$ & at $80 \mathrm{~kg} / \mathrm{s}$ & at $80 \mathrm{~kg} / \mathrm{s}$ & $\begin{array}{l}\text { number } \\
\text { @ } 130\end{array}$ & $\begin{array}{l}\text { number } \\
\text { @ } 130\end{array}$ & at $30 \mathrm{~kg} / \mathrm{s}$ & at $80 \mathrm{~kg} / \mathrm{s}$ & & & \\
\hline Name & Name & ${ }^{\circ} \mathrm{C}$ & & GJ Year & GJ Year & GJ Year & $\begin{array}{l}@ 1 \text { 130 } \\
\text { GJ/Year }\end{array}$ & $\begin{array}{l}@ 130 \\
\text { GJ/Year }\end{array}$ & MW el. & MW el. & $\mathrm{kJ} / \mathrm{kg}$ & & $\mathrm{km}$ \\
\hline Airdrie & $\mathrm{AB}$ & 60 & _- & _- & _- & _- & _- & _- & 0.11979 & & 159.72 & /Rundle dolstone & 2.3 \\
\hline Banff & $\mathrm{AB}$ & & - & - & - & - & - & - & - & _- & & Disturbed belt & \\
\hline Barrhead & $\mathrm{AB}$ & 35.2 & - & - & - & - & - & - & - & 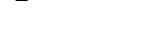 & $\overline{6} 0.6936$ & Mississippian & 1.1 \\
\hline Battleford & Sask. & & - & - & - & - & - & - & - & - & & shallow basin & \\
\hline Beaumont & $\mathrm{AB}$ & 40 & - & - & - & - & _- & - & & - & 79.86 & & $\overline{1} .2$ \\
\hline Blackfalds & $\mathrm{AB}$ & 59.5 & - & - & - & - & - & - & 0.113801 & ${ }^{-}-0.0126$ & 157.7235 & Banff l. & 1.75 \\
\hline Bonnyville & $\mathrm{AB}$ & - & $\begin{array}{l}- \\
-\end{array}$ & - & - & - & $\begin{array}{l}- \\
-\end{array}$ & - & - & - & - & shallow basin & - \\
\hline Brooks & $\mathrm{AB}$ & 30 & - & - & - & - & - & - & - & - & 39.93 & $\begin{array}{l}\text { /Rundle/Banff } \\
\text { carbonates }\end{array}$ & 1.1 \\
\hline Calgary & $\mathrm{AB}$ & 60 & - & - & - & - & - & - & 0.11979 & - & 159.72 & $\begin{array}{l}\text { /Rundle/Charles } \\
\text { carbonates }\end{array}$ & 2.4 \\
\hline Camrose & $\mathrm{AB}$ & 40 & - & - & _- & _- & - & _- & _- & - & 79.86 & Mississippian & 1.1 \\
\hline Canmore & $\mathrm{AB}$ & & - & - & - & - & - & - & - & - & & Disturbed belt & \\
\hline Cardston & $\mathrm{AB}$ & $\overline{50}$ & - & - & _- & _- & _- & _- & 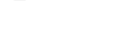 & & $\overline{1} 19.79$ & Banf/Rundle l. & 2.5 \\
\hline Carstairs & $A B$ & 55.2 & - & - & $\begin{array}{l}- \\
-\end{array}$ & - & - & $\begin{array}{lll}- \\
-\end{array}$ & 0.062291 & & 140.5536 & Banf 1./Rundle d. & 2.3 \\
\hline Chestermere & $\mathrm{AB}$ & 50 & - & - & - & - & - & $\begin{array}{lll}- \\
-\end{array}$ & - & _- & 119.79 & /Rundle dolstone & 2.1 \\
\hline Claresholm & $\mathrm{AB}$ & 50.4 & $\begin{array}{lll}- \\
-\end{array}$ & - & - & $\begin{array}{ll}- \\
-\end{array}$ & - & - & - & - & 121.3872 & Banf/Rundle l. & 2.4 \\
\hline Coaldale & $\mathrm{AB}$ & 30 & - & - & - & - & - & - & - & _- & 39.93 & Rundle 1. & 1.25 \\
\hline Cochrane & $\mathrm{AB}$ & - & - & - & - & - & - & - & - & - & - & Disturbed belt & - \\
\hline Cold Lake & $\mathrm{AB}$ & - & - & - & - & - & - & - & - & $\begin{array}{lll}- \\
-\end{array}$ & - & shallow basin & - \\
\hline Cold Lake & $\mathrm{AB}$ & - & & & & & & & & & - & shallow basin & - \\
\hline Dawson Creek & NE BC & $\overline{9} 1$ & $\overline{6} 8,313.36$ & $\overline{5} 3,891.08$ & 18,2169 & $143,709.6$ & $\overline{4} 14.5468$ & $\overline{1} 401.3$ & $\overline{0} .491139$ & $\overline{0} .7812$ & $\overline{2} 83.503$ & Mississippian & 2.6 \\
\hline Devon & $\mathrm{AB}$ & 51.3 & - & _- & - & - & _- & _- & & - & 124.9809 & Mississippian & 1.35 \\
\hline Didsbury & $A B$ & 57.5 & $\begin{array}{lll}- \\
-\end{array}$ & - & - & - & - & $\begin{array}{lll}- \\
-\end{array}$ & $\overline{0} .089842$ & $\begin{array}{lll}- \\
-\end{array}$ & 149.7375 & Banff l. & 2.3 \\
\hline Drayton Valley & $\mathrm{AB}$ & 60 & $\begin{array}{lll}- \\
-\end{array}$ & - & $\begin{array}{l}- \\
-\end{array}$ & - & - & $\begin{array}{lll}- \\
-\end{array}$ & 0.11979 & & 159.72 & Banff 1 . & 2 \\
\hline Drumheller & $\mathrm{AB}$ & 37.8 & $\begin{array}{lll}- \\
-\end{array}$ & $\begin{array}{ll}- \\
-\end{array}$ & $\begin{array}{l}- \\
-\end{array}$ & $\begin{array}{ll}- \\
-\end{array}$ & $\begin{array}{ll}- \\
-\end{array}$ & $\begin{array}{l}- \\
-\end{array}$ & & & 71.0754 & Mississippian & 1.4 \\
\hline E.Lloydminster & Sask. & & - & - & - & - & - & - & - & - & & shallow basin & \\
\hline Edmonton & $\mathrm{AB}$ & $\overline{4} 0$ & - & - & - & - & - & - & - & - & $\overline{7} 9.86$ & Mississippian & 1.2 \\
\hline Edson & $A B$ & 96.25 & $\overline{7} 9,882.56$ & $\overline{6} 3,017.8$ & $\overline{2} 13,020.2$ & $\overline{1} 68,047.5$ & $\overline{4} 84.7523$ & $\overline{1} 638.617$ & $\overline{0} .554029$ & $\overline{0} .9135$ & 304.4663 & Rundle 1. & 2.75 \\
\hline Estevan & Sask. & 57.6 & -5288.78 & - & - & - & - & - & - & - & 150.1368 & Mississippian & 1.6 \\
\hline Fort Liard NWT & NWT & 40 & - & - & - & - & - & - & - & - & - & Mississippian & 1 \\
\hline Fort Nelson & NE BC & & - & - & - & - & - & - & - & - & - & shallow basin & \\
\hline Fort Sask. & $\mathrm{AB}$ & $\overline{4} 0$ & - & $\begin{array}{lll}- \\
-\end{array}$ & - & - & - & $\begin{array}{lll}- \\
-\end{array}$ & $\begin{array}{l}- \\
-\end{array}$ & $\begin{array}{lll}- & & \end{array}$ & $\overline{7} 9.86$ & Mississippian & $\overline{1} .1$ \\
\hline Fort St. John & NE BC & 64 & $\overline{8} 814.627$ & $\overline{6} 953.688$ & $23,505.67$ & $\overline{1} 8,543.17$ & $\overline{5} 3.48991$ & $\overline{1} 80.8129$ & 0.167706 & 0.1008 & 175.692 & Mississippian & 2 \\
\hline Grand Centre & $\mathrm{AB}$ & - & - & - & - & - & - & - & - & - & - & shallow basin & - \\
\hline Grande Cache & $\mathrm{AB}$ & - & - & - & - & - & - & - & & & & Disturbed belt & - \\
\hline Grande Prairie & $\mathrm{AB}$ & 90 & $66,109.71$ & $52,152.66$ & $176,292.5$ & $139,073.8$ & 401.1743 & 1356.097 & 0.47916 & 0.756 & 279.51 & $\begin{array}{l}\text { /Rundle/Banff } \\
\text { carbonates }\end{array}$ & 1.9 \\
\hline High River & $\mathrm{AB}$ & 60 & - & - & - & - & - & - & 0.11979 & - & 159.72 & $\begin{array}{l}\text { /Rundle/Banff } \\
\text { carbonates }\end{array}$ & 2.4 \\
\hline
\end{tabular}


Table A4. Cont.

\begin{tabular}{|c|c|c|c|c|c|c|c|c|c|c|c|c|c|}
\hline City Location & Province & Temperature $\mathrm{T}$ & Energy 1 & Energy 2 & Energy 3 & Energy 4 & Households & Households & Power & Power & $\begin{array}{l}\text { Enthalpy } \\
\text { Gain }\end{array}$ & Formation Group & Depth \\
\hline & & & at $C=3993$ & at $C=3150$ & at $C=3993$ & at $C=3150$ & Minimum & Maximum & at $C=3993$ & at $C=3150$ & at $C=3993$ & $\begin{array}{l}\text { top of } \\
\text { Winterburn } \\
\text { Group }\end{array}$ & \\
\hline Name & Name & ${ }^{\circ} \mathrm{C}$ & $\begin{array}{l}\text { at } 30 \mathrm{~kg} / \mathrm{s} \\
\text { GJ Year }\end{array}$ & $\begin{array}{l}\text { at } 30 \mathrm{~kg} / \mathrm{s} \\
\text { GJ Year }\end{array}$ & $\begin{array}{l}\text { at } 80 \mathrm{~kg} / \mathrm{s} \\
\text { GJ Year }\end{array}$ & $\begin{array}{l}\text { at } 80 \mathrm{~kg} / \mathrm{s} \\
\text { GJ Year }\end{array}$ & $\begin{array}{l}\text { number } \\
@ \text { 130 } \\
\text { GJ/Year }\end{array}$ & $\begin{array}{l}\text { number } \\
@ \text { 130 } \\
\text { GI/Year }\end{array}$ & $\begin{array}{l}\text { at } 30 \mathrm{~kg} / \mathrm{s} \\
\text { MW el. }\end{array}$ & $\begin{array}{l}\text { at } 80 \mathrm{~kg} / \mathrm{s} \\
\mathrm{MW} \mathrm{el.}\end{array}$ & $\mathrm{kJ} / \mathrm{kg}$ & & km \\
\hline Hinton & $\mathrm{AB}$ & 135 & $165,274.3$ & $130,381.7$ & $440,731.4$ & $347,684.4$ & 1002.936 & 3390.241 & 1.018215 & 1.89 & 459.195 & $\begin{array}{l}\text { /Turney } \\
\text { Valley/Elkton }\end{array}$ & $3-4.5$ \\
\hline $\begin{array}{l}\text { Humboldt } \\
\text { Innisfail }\end{array}$ & $\begin{array}{l}\text { Sask. } \\
\text { AB }\end{array}$ & $\overline{5} 0$ & - & - & - & - & - & - & - & - & $\overline{1} 19.79$ & $\begin{array}{l}\text { shallow basin } \\
\text { Banff } 1 \text {. }\end{array}$ & $\overline{2}$ \\
\hline Jasper & $\mathrm{AB}$ & - & - & - & - & - & - & - & - & - & - & Disturbed belt & - \\
\hline $\begin{array}{l}\text { Kindersley } \\
\text { Lacombe }\end{array}$ & $\begin{array}{l}\text { Sask. } \\
\text { AB }\end{array}$ & $\overline{70}$ & $\overline{22}, 036.57$ & $\overline{17}, 384.22$ & $\overline{5} 8,764.18$ & $\overline{4} 6,357.92$ & $\overline{1} 33.7248$ & $\overline{4} 52.0322$ & $\overline{0} .23958$ & $\overline{0} .252$ & $\overline{1} 99.65$ & shallow basin & -17 \\
\hline Langdon & $\mathrm{AB}$ & 46 & $20,000.01$ & - & $-30,07.10$ & 70, & $100.7<+0$ & +52.0024 & 0.20700 & 0.202 & $\begin{array}{l}193.03 \\
103.818\end{array}$ & $\begin{array}{l}\text { Mississippian } \\
\text { Rundle d }\end{array}$ & $\frac{1.7}{2}$ \\
\hline $\begin{array}{l}\text { Leduc } \\
\text { L }\end{array}$ & $\mathrm{AB}$ & 55 & $\begin{array}{lll}- & & \\
-\end{array}$ & $\begin{array}{lll}- & & \end{array}$ & - & - & $\begin{array}{lll}- & & \\
-\end{array}$ & $\begin{array}{lll}- & & \\
-\end{array}$ & $\begin{array}{lll}- & & \\
-\end{array}$ & - & 139.755 & Mississippian & 1.3 \\
\hline Lethbridge & $A B$ & 35 & - & - & - & - & - & - & $\begin{array}{lll}- \\
-\end{array}$ & $\begin{array}{lll}- \\
-\end{array}$ & 59.895 & Runde limestone & 1.4 \\
\hline Lloydminster & $\mathrm{AB}$ & _- & - & - & - & - & - & - & - & - & - & shallow basin & - \\
\hline Martensville & Sask. & $\begin{array}{l}- \\
-\end{array}$ & $\begin{array}{l}- \\
-\end{array}$ & $\begin{array}{l}- \\
-\end{array}$ & - & - & - & $\begin{array}{lll}- \\
-\end{array}$ & $\begin{array}{l}- \\
-\end{array}$ & $\begin{array}{l}- \\
-\end{array}$ & - & shallow basin & $\begin{array}{l}- \\
-\end{array}$ \\
\hline Meadow Lake & Sask. & - & - & - & - & - & - & - & - & - & - & shallow basin & - \\
\hline Medicine Hat & Sask. & 30 & - & - & - & - & - & - & - & - & 39.93 & $\begin{array}{l}\text { /Rundle/Banff } \\
\text { carbonates }\end{array}$ & - \\
\hline Melford & $\mathrm{AB}$ & - & - & - & - & - & - & - & - & - & _- & shallow basin & - \\
\hline Melville & Sask. & $\overline{30}$ & -66.1097 & - & - & - & - & - & - & - & $-\overline{39} 93$ & shallow basin & -12 \\
\hline $\begin{array}{l}\text { Moose Jaw } \\
\text { Morinville }\end{array}$ & $\mathrm{AB}$ & 40 & $\begin{array}{l}-06,109.7 \\
-\end{array}$ & $\begin{array}{l}- \\
-\end{array}$ & - & $\begin{array}{l}- \\
-\end{array}$ & $\begin{array}{l}- \\
-\end{array}$ & - & $\begin{array}{l}- \\
-\end{array}$ & $\begin{array}{l}- \\
-\end{array}$ & $\begin{array}{l}39.93 \\
79.86\end{array}$ & $\begin{array}{l}\text { Mississippian } \\
\text { Mississippian }\end{array}$ & 1.1 \\
\hline $\begin{array}{l}\text { North } \\
\text { Battleford }\end{array}$ & Sask. & _- & - & - & _ & _- & - & - & - & _- & - & shallow basin & - \\
\hline Olds & $\mathrm{AB}$ & 57.5 & _- & - & - & _- & - & - & 0.089842 & _- & 149.7375 & Mississippian & 2.3 \\
\hline Peace River & $\mathrm{AB}$ & & - & - & - & - & - & - & _- & - & & shallow basin & \\
\hline Penhold & $A B$ & $\overline{5} 0.4$ & - & - & - & - & - & - & - & - & 121.3872 & Banf 1. & $\overline{1} .8$ \\
\hline Pincher Creek & $\mathrm{AB}$ & & - & - & - & - & - & - & - & - & & Disturbed belt & \\
\hline Ponoka & $\mathrm{AB}$ & $\overline{6} 4$ & $\overline{8} 814.627$ & $\overline{6} 953.688$ & $\overline{2} 3,505.67$ & $\overline{1} 8,543.17$ & $\overline{5} 3.48991$ & $\overline{1} 80.8129$ & $\overline{0} .167706$ & $\overline{0} .1008$ & 175.692 & Mississippian & $\overline{1} .6$ \\
\hline Prince Albert & Sask. & & - & - & - & - & & & _- & & & shallow basin & \\
\hline Raymond & $\mathrm{AB}$ & 31.2 & _- & _- & - & - & - & - & - & - & $\overline{4} 4.7216$ & Rundle 1. & $\overline{1} .3$ \\
\hline Red Deer & $\mathrm{AB}$ & 60 & $\begin{array}{lll}- \\
-\end{array}$ & $\begin{array}{l}- \\
-\end{array}$ & $\begin{array}{lll}- \\
-\end{array}$ & - & $\begin{array}{l}- \\
-\end{array}$ & - & $\overline{0} .11979$ & $\begin{array}{lll}- \\
-\end{array}$ & 159.72 & /Banff limestone & 1.8 \\
\hline Redcliff & $\mathrm{AB}$ & & & $\begin{array}{l}- \\
-\end{array}$ & $\begin{array}{lll}- \\
-\end{array}$ & - & $\begin{array}{l}- \\
-\end{array}$ & - & & $\begin{array}{lll}- \\
-\end{array}$ & & shallow basin & \\
\hline Regina & Sask. & $\overline{3} 0$ & ${ }^{-} 66109.7$ & - & $\begin{array}{l}- \\
-\end{array}$ & $\begin{array}{l}- \\
-\end{array}$ & $\begin{array}{l}- \\
-\end{array}$ & $\begin{array}{l}- \\
-\end{array}$ & $\begin{array}{l}- \\
-\end{array}$ & $\begin{array}{l}- \\
-\end{array}$ & $\overline{3} 9.93$ & Mississippian & $\overline{1}$ \\
\hline $\begin{array}{l}\text { Rocky } \\
\text { Mountain } \\
\text { House }\end{array}$ & $\mathrm{AB}$ & 81 & $46,276.79$ & $36,506.86$ & $123,404.8$ & $97,351.63$ & 280.822 & 949.2676 & 0.371349 & 0.5292 & 243.573 & Rundle 1/Banff 1. & 2.7 \\
\hline Saskatoon & Sask. & _- & _- & _- & _- & _- & _- & _- & _- & _- & - & shallow basin & _- \\
\hline Slave Lake & $\mathrm{AB}$ & & - & - & - & - & - & - & - & - & - & shallow basin & \\
\hline Spruce Grove & $\mathrm{AB}$ & 45 & - & - & - & _- & - & _- & _- & _- & 99.825 & Mississippian & 1.3 \\
\hline St. Albert & $\mathrm{AB}$ & 45 & - & - & - & - & - & - & - & - & 99.825 & Mississippian & 1.2 \\
\hline St. Paul & $\mathrm{AB}$ & & $\begin{array}{lll}- \\
-\end{array}$ & $\begin{array}{l}- \\
-\end{array}$ & $\begin{array}{ll}- \\
-\end{array}$ & $\begin{array}{lll}- \\
-\end{array}$ & $\begin{array}{lll}- \\
-\end{array}$ & $\begin{array}{l}- \\
-\end{array}$ & $\begin{array}{lll}- \\
-\end{array}$ & $\begin{array}{lll}- \\
-\end{array}$ & & shallow basin & \\
\hline Stettler & $\mathrm{AB}$ & $\overline{3} 7.7$ & - & - & - & - & - & - & - & - & $\overline{7} 0.6761$ & Mississippian & $\overline{1} .3$ \\
\hline Stony Plain & $\mathrm{AB}$ & 50 & - & - & - & - & - & - & - & - & 119.79 & Mississippian & 1.4 \\
\hline Swift Current & Sask. & 31.05 & $-63,795.9$ & $\begin{array}{lll}- \\
-\end{array}$ & $\begin{array}{lll}- \\
-\end{array}$ & $\begin{array}{lll}- \\
-\end{array}$ & $\begin{array}{lll}- \\
-\end{array}$ & $\begin{array}{lll}- \\
-\end{array}$ & $\begin{array}{lll}- \\
-\end{array}$ & $\begin{array}{lll}- \\
-\end{array}$ & 44.12265 & Mississippian & 1.15 \\
\hline
\end{tabular}


Table A4. Cont.

\begin{tabular}{|c|c|c|c|c|c|c|c|c|c|c|c|c|c|}
\hline City Location & Province & Temperature $\mathrm{T}$ & Energy 1 & Energy 2 & Energy 3 & Energy 4 & Households & Households & Power & Power & $\begin{array}{l}\text { Enthalpy } \\
\text { Gain }\end{array}$ & Formation Group & Depth \\
\hline Name & Name & ${ }^{\circ} \mathrm{C}$ & $\begin{array}{l}\text { at } \mathrm{C}=3993 \\
\text { at } 30 \mathrm{~kg} / \mathrm{s} \\
\text { GJ Year }\end{array}$ & $\begin{array}{l}\text { at } C=3150 \\
\text { at } 30 \mathrm{~kg} / \mathrm{s} \\
\text { GJ Year }\end{array}$ & $\begin{array}{l}\text { at } C=3993 \\
\text { at } 80 \mathrm{~kg} / \mathrm{s} \\
\text { GJ Year }\end{array}$ & $\begin{array}{l}\text { at } C=3150 \\
\text { at } 80 \mathrm{~kg} / \mathrm{s} \\
\text { GJ Year }\end{array}$ & $\begin{array}{l}\text { Minimum } \\
\text { number } \\
@ 130 \\
\text { GJ/Year } \\
\end{array}$ & $\begin{array}{l}\text { Maximum } \\
\text { number } \\
@ 130 \\
\text { GJ/Year } \\
\end{array}$ & $\begin{array}{l}\text { at } C=3993 \\
\text { at } 30 \mathrm{~kg} / \mathrm{s} \\
\text { MW el. }\end{array}$ & $\begin{array}{l}\text { at } C=3150 \\
\text { at } 80 \mathrm{~kg} / \mathrm{s} \\
\text { MW el. }\end{array}$ & at $C=3993$ & $\begin{array}{l}\text { top of } \\
\text { Winterburn } \\
\text { Group }\end{array}$ & km \\
\hline Taber & $\mathrm{AB}$ & 27 & - & _- & _- & _- & _- & _- & _- & _- & 27.951 & Rundle 1. & 1 \\
\hline $\begin{array}{l}\text { Three } \\
\text { Hills+M8A6:M41 }\end{array}$ & $\mathrm{AB}$ & 41.6 & - & - & - & - & - & - & & & 86.2488 & Rundle d. & 1.6 \\
\hline Vegreville & Sask. & - & - & - & - & - & - & - & - & - & - & shallow basin & - \\
\hline Vermilion & $\mathrm{AB}$ & - & - & - & - & - & - & - & & & & shallow basin & - \\
\hline Virden Man. & Man. & - & - & - & - & - & - & - & - & - & - & shallow basin & - \\
\hline Wainwright & $\mathrm{AB}$ & - & - & - & - & - & - & - & - & - & - & shallow basin & - \\
\hline Warman & Sask. & - & - & - & - & - & - & - &  & - & - & shallow basin & - \\
\hline Westlock & $\mathrm{AB}$ & - & - & - & - & - & - & _- & & - & & shallow basin & $\begin{array}{lll}- \\
-\end{array}$ \\
\hline Wetaskiwin & $\mathrm{AB}$ & $\overline{6} 0$ & - & - & - & - & $\begin{array}{ll}- \\
-\end{array}$ & $\begin{array}{lll}- \\
-\end{array}$ & $\overline{0} .11979$ & $\begin{array}{lll}- \\
-\end{array}$ & $\overline{1} 59.72$ & Mississippian & $\overline{1} .4$ \\
\hline Weyburn & Sask. & 44.2 & ${ }^{-} 34,817.8$ & $\begin{array}{lll}- \\
-\end{array}$ & $\begin{array}{l}- \\
-\end{array}$ & $\begin{array}{lll}- \\
-\end{array}$ & $\begin{array}{ll}- \\
-\end{array}$ & $\begin{array}{lll}- \\
-\end{array}$ & - & $\begin{array}{lll}- & & \\
-\end{array}$ & 96.6306 & Mississippian & 1.3 \\
\hline White City & Sask. & 30 & $-66,109.7$ & $\begin{array}{ll}- \\
-\end{array}$ & & & & & - & & 39.93 & Mississippian & 1 \\
\hline Whitecourt & $\mathrm{AB}$ & 70 & $22,036.57$ & $\overline{1} 7,384.22$ & $\overline{5} 8,764.18$ & $\overline{4} 6,357.92$ & $\overline{1} 33.7248$ & $\overline{4} 52.0322$ & $\overline{0} .23958$ & $\overline{0} .252$ & 199.65 & / Banff limestone & 1.8 \\
\hline Yorkton & Sask. & _- & - & - & - & - & - & - & - & - & - & shallow basin & - \\
\hline
\end{tabular}


feasible.

\begin{tabular}{|c|c|c|c|c|c|c|c|c|c|c|c|c|c|}
\hline City Location & Province & Temperature $T$ & Energy 1 & Energy 2 & Energy 3 & Energy 4 & Households & Households & Power & Power & $\begin{array}{l}\text { Enthalpy } \\
\text { Gain }\end{array}$ & Formation Group & Depth \\
\hline & & & $\begin{array}{l}\text { at } C=3993 \\
\text { at } 30 \mathrm{~kg} / \mathrm{s}\end{array}$ & $\begin{array}{l}\text { at } C=3150 \\
\text { at } 30 \mathrm{~kg} / \mathrm{s}\end{array}$ & $\begin{array}{l}\text { at } C=3993 \\
\text { at } 80 \mathrm{~kg} / \mathrm{s}\end{array}$ & $\begin{array}{l}\text { at } C=3150 \\
\text { at } 80 \mathrm{~kg} / \mathrm{s}\end{array}$ & $\begin{array}{l}\text { Minimum } \\
\text { number }\end{array}$ & $\begin{array}{l}\text { Maximum } \\
\text { number }\end{array}$ & $\begin{array}{l}\text { at } C=3993 \\
\text { at } 30 \mathrm{~kg} / \mathrm{s}\end{array}$ & $\begin{array}{l}\text { at } C=3150 \\
\text { at } 80 \mathrm{~kg} / \mathrm{s}\end{array}$ & at $C=3993$ & Lower Cretaceous & \\
\hline Name & Name & ${ }^{\circ} \mathrm{C}$ & GJ Year & GJ Year & GJ Year & GJ Year & $\begin{array}{l}\text { @ 130 } \\
\text { GJ/Year }\end{array}$ & $\begin{array}{l}@ 130 \\
\text { GJ/Year }\end{array}$ & MW el. & MW el. & $\mathrm{kJ} / \mathrm{kg}$ & & $\mathrm{km}$ \\
\hline Airdrie & $\mathrm{AB}$ & 45 & - & - & - & - & - & - & - & - & 99.825 & $\begin{array}{l}\text { Lower Mannville } \\
\text { Group }\end{array}$ & 2.3 \\
\hline Banff & $\mathrm{AB}$ & - & - & - & - & - & - & - & _- & - & - & Disturbed belt & \\
\hline Barrhead & $\mathrm{AB}$ & 38.4 & - & - & - & - & - & - & - & - & 73.4712 & $\begin{array}{l}\text { Lower Mannville } \\
\text { Group }\end{array}$ & 1.2 \\
\hline Battleford & Sask. & - & _- & - & _- & - & _- & _- & _- & _- & _- & shallow basin & _- \\
\hline Beaumont & $\mathrm{AB}$ & 30 & - & - & - & - & - & - & - & - & 39.93 & $\begin{array}{l}\text { Lower Mannville } \\
\text { Group }\end{array}$ & 1.2 \\
\hline Blackfalds & $\mathrm{AB}$ & 63 & 6610.971 & 5215.266 & $17,629.25$ & $13,907.38$ & 40.11743 & 135.6097 & 0.155727 & 0.0756 & 171.699 & $\begin{array}{l}\text { Lower Mannville } \\
\text { Group }\end{array}$ & 1.75 \\
\hline Bonnyville & $\mathrm{AB}$ & - & - & - & - & - & - & - & - & - & - & shallow basin & _- \\
\hline Brooks & $\mathrm{AB}$ & 20 & - & - & - & - & - & - & - & - & 0 & $\begin{array}{l}\text { Lower Mannville } \\
\text { Group }\end{array}$ & 1.1 \\
\hline
\end{tabular}


Table A5. Cont.

\begin{tabular}{|c|c|c|c|c|c|c|c|c|c|c|c|c|c|}
\hline City Location & Province & Temperature T & Energy 1 & Energy 2 & Energy 3 & Energy 4 & Households & Households & Power & Power & $\begin{array}{l}\text { Enthalpy } \\
\text { Gain }\end{array}$ & Formation Group & Depth \\
\hline & & & $\begin{array}{l}\text { at } C=3993 \\
\text { at } 30 \mathrm{~kg} / \mathrm{s}\end{array}$ & $\begin{array}{l}\text { at } C=3150 \\
\text { at } 30 \mathrm{~kg} / \mathrm{s}\end{array}$ & $\begin{array}{l}\text { at } C=3993 \\
\text { at } 80 \mathrm{~kg} / \mathrm{s}\end{array}$ & $\begin{array}{l}\text { at } C=3150 \\
\text { at } 80 \mathrm{~kg} / \mathrm{s}\end{array}$ & $\begin{array}{l}\text { Minimum } \\
\text { number }\end{array}$ & $\begin{array}{l}\text { Maximum } \\
\text { number }\end{array}$ & $\begin{array}{l}\text { at } C=3993 \\
\text { at } 30 \mathrm{~kg} / \mathrm{s}\end{array}$ & $\begin{array}{l}\text { at } C=3150 \\
\text { at } 80 \mathrm{~kg} / \mathrm{s}\end{array}$ & at $\mathrm{C}=3993$ & Lower Cretaceous & \\
\hline Name & Name & ${ }^{\circ} \mathrm{C}$ & GJ Year & GJ Year & GJ Year & GJ Year & $\begin{array}{l}\text { @ 130 } \\
\text { GJ/Year }\end{array}$ & $\begin{array}{l}\text { @ 130 } \\
\text { GJ/Year }\end{array}$ & MW el. & MW el. & $\mathrm{kJ} / \mathrm{kg}$ & & km \\
\hline Calgary & $\mathrm{AB}$ & 50 & - & - & - & - & - & - & - & - & 119.79 & $\begin{array}{l}\text { Lower Mannville } \\
\text { Group }\end{array}$ & 2.4 \\
\hline Camrose & $\mathrm{AB}$ & 30 & - & - & - & - & - & - & - & - & 39.93 & $\begin{array}{l}\text { Lower Mannville } \\
\text { Group }\end{array}$ & 1.1 \\
\hline Canmore & $\mathrm{AB}$ & _- & _- & - & _- & - & - & - & - & - & - & Disturbed belt & - \\
\hline Cardston & $\mathrm{AB}$ & - & - & - & - & - & - & - & - & - & - & Disturbed belt & - \\
\hline Carstairs & $\mathrm{AB}$ & - & - & - & - & - & - & - & - & - & 149.7375 & $\begin{array}{l}\text { Lower Mannville } \\
\text { Group }\end{array}$ & 2.3 \\
\hline Chestermere & $\mathrm{AB}$ & 40 & - & - & - & - & - & - & - & - & 79.86 & $\begin{array}{l}\text { Lower Mannville } \\
\text { Group }\end{array}$ & 2.1 \\
\hline Claresholm & $\mathrm{AB}$ & - & - & - & - & - & - & - & - & - & 121.3872 & $\begin{array}{l}\text { Lower Mannville } \\
\text { Group }\end{array}$ & 2.4 \\
\hline Coaldale & $\mathrm{AB}$ & 32.5 & - & - & - & _- & - & - & - & - & 49.9125 & $\begin{array}{l}\text { Lower Mannville } \\
\text { Group }\end{array}$ & 1.3 \\
\hline Cochrane & $\mathrm{AB}$ & - & - & - & - & - & - & - & - & - & - & Disturbed belt & - \\
\hline Cold Lake & $\mathrm{AB}$ & - & - & - & - & - & - & - & - & - & - & shallow basin & - \\
\hline Cold Lake & $\mathrm{AB}$ & - & - & - & $\begin{array}{lll}- \\
-\end{array}$ & - & - & $\begin{array}{l}- \\
-\end{array}$ & - & - & - & shallow basin & - \\
\hline Dawson Creek & NE BC & 56 & - & - & - & - & - & - & - & - & 143.748 & $\begin{array}{l}\text { Lower Mannville } \\
\text { Group }\end{array}$ & 1.6 \\
\hline Devon & $\mathrm{AB}$ & 51.8 & - & - & - & - & - & - & - & - & 126.9774 & $\begin{array}{l}\text { Lower Mannville } \\
\text { Group }\end{array}$ & 1.4 \\
\hline Didsbury & $\mathrm{AB}$ & 57.5 & & & & & & & 0.089842 & & 149.7375 & $\begin{array}{l}\text { Lower Mannville } \\
\text { Group }\end{array}$ & 2.3 \\
\hline Drayton Valley & $\mathrm{AB}$ & 68.25 & $18,180.17$ & $14,341.98$ & $48,480.45$ & $38,245.28$ & 110.3229 & 372.9265 & 0.218617 & 0.2079 & 192.6623 & $\begin{array}{l}\text { Lower Mannville } \\
\text { Group }\end{array}$ & 1.95 \\
\hline Drumheller & $\mathrm{AB}$ & 37.8 & - & - & - & - & - & - & - & - & 71.0754 & $\begin{array}{l}\text { Lower Mannville } \\
\text { Group }\end{array}$ & 1.4 \\
\hline E.Lloydminster & Sask. & - & - & - & _- & - & - & - & - & - & - & shallow basin & - \\
\hline Edmonton & $\mathrm{AB}$ & 35 & - & - & - & - & - & - & - & - & 59.895 & $\begin{array}{l}\text { Lower Mannville } \\
\text { Group }\end{array}$ & 1.2 \\
\hline Edson & $\mathrm{AB}$ & 91 & $68,313.36$ & $53,891.08$ & 182,169 & $143,709.6$ & 414.5468 & 1401.3 & 0.491139 & 0.7812 & 283.503 & $\begin{array}{l}\text { Lower Mannville } \\
\text { Group }\end{array}$ & 2.6 \\
\hline Estevan & Sask. & 46.8 & - & - & - & - & - & - & - & - & 107.0124 & shallow basin & 1.3 \\
\hline Fort Liard NWT & NWT & 40 & - & - & - & - & - & - & - & - & - & shallow basin & 1 \\
\hline Fort Nelson & NE BC & - & - & - & - & - & - & - & - & - & - & shallow basin & - \\
\hline Fort Sask. & $\mathrm{AB}$ & 30 & - & - & - & - & - & - & - & - & 39.93 & $\begin{array}{l}\text { Lower Mannville } \\
\text { Group }\end{array}$ & 1.1 \\
\hline Fort St. John & NE BC & 43.2 & - & - & - & - & - & - & - & - & 92.6376 & $\begin{array}{l}\text { Lower Mannville } \\
\text { Group }\end{array}$ & 1.35 \\
\hline $\begin{array}{l}\text { Grand Centre } \\
\text { Grande Cache }\end{array}$ & $\begin{array}{l}\mathrm{AB} \\
\mathrm{AB}\end{array}$ & - & $\begin{array}{l}- \\
-\end{array}$ & $\begin{array}{l}- \\
-\end{array}$ & - & $\begin{array}{l}- \\
-\end{array}$ & $\begin{array}{l}- \\
-\end{array}$ & $\begin{array}{l}- \\
-\end{array}$ & $\begin{array}{l}- \\
-\end{array}$ & $\begin{array}{l}- \\
-\end{array}$ & $\begin{array}{l}- \\
-\end{array}$ & $\begin{array}{l}\text { shallow } \\
\text { Disturbed belt }\end{array}$ & - \\
\hline Grande Prairie & $\mathrm{AB}$ & 70 & $22,036.57$ & $17,384.22$ & $58,764.18$ & $46,357.92$ & 133.7248 & 452.0322 & 0.23958 & 0.252 & 199.65 & $\begin{array}{l}\text { Lower Man- } \\
\text { nville/Cadominium }\end{array}$ & 1.9 \\
\hline
\end{tabular}


Table A5. Cont.

\begin{tabular}{|c|c|c|c|c|c|c|c|c|c|c|c|c|c|}
\hline City Location & Province & Temperature T & Energy 1 & Energy 2 & Energy 3 & Energy 4 & Households & Households & Power & Power & $\begin{array}{l}\text { Enthalpy } \\
\text { Gain }\end{array}$ & Formation Group & Depth \\
\hline & & & $\begin{array}{l}\text { at } C=3993 \\
\text { at } 30 \mathrm{~kg} / \mathrm{s}\end{array}$ & $\begin{array}{l}\text { at } C=3150 \\
\text { at } 30 \mathrm{~kg} / \mathrm{s}\end{array}$ & $\begin{array}{l}\text { at } C=3993 \\
\text { at } 80 \mathrm{~kg} / \mathrm{s}\end{array}$ & $\begin{array}{l}\text { at } C=3150 \\
\text { at } 80 \mathrm{~kg} / \mathrm{s}\end{array}$ & $\begin{array}{l}\text { Minimum } \\
\text { number }\end{array}$ & $\begin{array}{l}\text { Maximum } \\
\text { number }\end{array}$ & $\begin{array}{l}\text { at } C=3993 \\
\text { at } 30 \mathrm{~kg} / \mathrm{s}\end{array}$ & $\begin{array}{l}\text { at } C=3150 \\
\text { at } 80 \mathrm{~kg} / \mathrm{s}\end{array}$ & at $\mathrm{C}=3993$ & Lower Cretaceous & \\
\hline Name & Name & ${ }^{\circ} \mathrm{C}$ & GJ Year & GJ Year & GJ Year & GJ Year & $\begin{array}{l}\text { @ 130 } \\
\text { GJ/Year }\end{array}$ & $\begin{array}{l}\text { @ } 130 \\
\text { GJ/Year }\end{array}$ & MW el. & MW el. & $\mathrm{kJ} / \mathrm{kg}$ & & $\mathrm{km}$ \\
\hline High River & $\mathrm{AB}$ & 50 & - & - & - & - & - & - & - & - & 119.79 & $\begin{array}{l}\text { Lower Mannville } \\
\text { Group }\end{array}$ & 2.4 \\
\hline Hinton & $\mathrm{AB}$ & 120 & $132,219.4$ & $104,305.3$ & $352,585.1$ & $278,147.5$ & 802.3486 & 2712.193 & 0.83853 & 1.512 & 399.3 & $\begin{array}{l}\text { Lower Man- } \\
\text { nville/Cadominium }\end{array}$ & 4 \\
\hline Humboldt & Sask. & - & - & - & - & - & - & - & - & - & - & shallow basin & - \\
\hline Innisfail & $\mathrm{AB}$ & 50 & - & - & - & - & - & - & - & - & 119.79 & $\begin{array}{l}\text { Lower Mannville } \\
\text { Group }\end{array}$ & 2 \\
\hline Jasper & $\mathrm{AB}$ & & - & - & - & - & - & - & - & - & & Disturbed belt & - \\
\hline Kindersley & Sask. & - & - & - & - & - & - & - & - & - & - & shallow basin & - \\
\hline Lacombe & $\mathrm{AB}$ & 60 & - & - & - & - & - & - & - & - & 159.72 & $\begin{array}{l}\text { Lower Mannville } \\
\text { Group }\end{array}$ & 1.7 \\
\hline Langdon & $\mathrm{AB}$ & 43.7 & & & & & & & & & 94.6341 & $\begin{array}{l}\text { Lower Mannville } \\
\text { Group }\end{array}$ & 1.9 \\
\hline Leduc & $\mathrm{AB}$ & 45 & - & - & - & - & - & - & - & - & 99.825 & $\begin{array}{l}\text { Lower Mannville } \\
\text { Group }\end{array}$ & 1.3 \\
\hline Lethbridge & $\mathrm{AB}$ & 30 & - & - & - & - & - & - & - & - & 39.93 & $\begin{array}{l}\text { Lower Man- } \\
\text { nville/Cadominium }\end{array}$ & 1.4 \\
\hline Lloydminster & $\mathrm{AB}$ & - & - & - & - & - & - & - & - & - & _- & shallow basin & 0 \\
\hline Malville & Sask. & - & - & - & - & - & - & - & - & - & - & shallow basin & - \\
\hline $\begin{array}{l}\text { Martensville } \\
\text { Meadow Lake }\end{array}$ & $\begin{array}{l}\text { Sask. } \\
\text { Sask. }\end{array}$ & - & - & - & - & - & - & - & - & - & - & shallow basin & - \\
\hline $\begin{array}{l}\text { Meadow Lake } \\
\text { Medicine Hat }\end{array}$ & Sask. & - & - & - & - & - & - & - & - & - & - & shallow basin & - \\
\hline $\begin{array}{l}\text { Medicine Hat } \\
\text { Melford }\end{array}$ & $\begin{array}{l}\text { AB } \\
\text { Sask. }\end{array}$ & - & - & - & - & - & - & - & - & - & - & shallow basin & - \\
\hline $\begin{array}{l}\text { Melford } \\
\text { Moose Jaw }\end{array}$ & Sask. & $\overline{25}$ & - & - & - & - & - & - & - & - & $\overline{1} 9965$ & shallow basin & $\overline{1}$ \\
\hline Morinville & $\mathrm{AB}$ & 30 & - & - & - & - & - & $\begin{array}{l}- \\
-\end{array}$ & - & $\begin{array}{l}- \\
-\end{array}$ & $\begin{array}{l}19.963 \\
39.93\end{array}$ & $\begin{array}{l}\text { shallow basin } \\
\text { Lower Mannville }\end{array}$ & 1.1 \\
\hline $\begin{array}{l}\text { North } \\
\text { Battleford }\end{array}$ & Sask. & - & - & - & - & 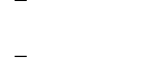 & - & 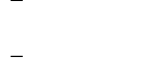 & - & - & - & $\begin{array}{l}\text { Group } \\
\text { shallow basin }\end{array}$ & - \\
\hline Olds & $\mathrm{AB}$ & 57.5 & - & - & - & - & - & - & - & - & 149.7375 & $\begin{array}{l}\text { Lower Mannville } \\
\text { Group }\end{array}$ & 2.3 \\
\hline Peace River & $\mathrm{AB}$ & - & _- & - & - & - & - & - & - & - & - & shallow basin & \\
\hline Penhold & $\mathrm{AB}$ & - & - & - & - & - & - & - & - & - & 140.1543 & $\begin{array}{l}\text { Lower Mannville } \\
\text { Group }\end{array}$ & 1.9 \\
\hline Pincher Creek & $\mathrm{AB}$ & - & - & - & _- & - & - & - & _- & - & - & Disturbed belt & - \\
\hline Ponoka & $\mathrm{AB}$ & 65.6 & $12,340.48$ & 9735.163 & $32,907.94$ & $25,960.44$ & 74.88587 & 253.138 & 0.186872 & 0.14112 & 182.0808 & $\begin{array}{l}\text { Lower Mannville } \\
\text { Group }\end{array}$ & 1.6 \\
\hline Prince Albert & Sask. & - & - & - & - & - & - & - & - & - & - & shallow basin & - \\
\hline Raymond & $\mathrm{AB}$ & - & - & - & - & - & - & - & - & - & 49.5132 & $\begin{array}{l}\text { Lower Mannville } \\
\text { Group }\end{array}$ & 1.35 \\
\hline Red Deer & $\mathrm{AB}$ & 50 & - & - & - & - & - & - & - & - & 119.79 & $\begin{array}{l}\text { Lower Mannville } \\
\text { Group }\end{array}$ & 1.8 \\
\hline
\end{tabular}


Table A5. Cont.

\begin{tabular}{|c|c|c|c|c|c|c|c|c|c|c|c|c|c|}
\hline City Location & Province & Temperature $\mathrm{T}$ & Energy 1 & Energy 2 & Energy 3 & Energy 4 & Households & Households & Power & Power & $\begin{array}{l}\text { Enthalpy } \\
\text { Gain }\end{array}$ & Formation Group & Depth \\
\hline & & & $\begin{array}{l}\text { at } C=3993 \\
\text { at } 30 \mathrm{~kg} / \mathrm{s}\end{array}$ & $\begin{array}{l}\text { at } C=3150 \\
\text { at } 30 \mathrm{~kg} / \mathrm{s}\end{array}$ & $\begin{array}{l}\text { at } C=3993 \\
\text { at } 80 \mathrm{~kg} / \mathrm{s}\end{array}$ & $\begin{array}{l}\text { at } C=3150 \\
\text { at } 80 \mathrm{~kg} / \mathrm{s}\end{array}$ & $\begin{array}{l}\text { Minimum } \\
\text { number }\end{array}$ & $\begin{array}{l}\text { Maximum } \\
\text { number }\end{array}$ & $\begin{array}{l}\text { at } C=3993 \\
\text { at } 30 \mathrm{~kg} / \mathrm{s}\end{array}$ & $\begin{array}{l}\text { at } C=3150 \\
\text { at } 80 \mathrm{~kg} / \mathrm{s}\end{array}$ & at $C=3993$ & Lower Cretaceous & \\
\hline Name & Name & ${ }^{\circ} \mathrm{C}$ & GJ Year & GJ Year & GJ Year & GJ Year & $\begin{array}{l}\text { @ 130 } \\
\text { GJ/Year }\end{array}$ & $\begin{array}{l}\text { @ 130 } \\
\text { GJ/Year }\end{array}$ & MW el. & MW el. & $\mathrm{kJ} / \mathrm{kg}$ & & km \\
\hline Redcliff & $\mathrm{AB}$ & & - & - & - & - & - & - & - & - & - & shallow basin & - \\
\hline Regina & Sask. & - & - & - & - & - & - & - & - & - & - & shallow basin & - \\
\hline $\begin{array}{l}\text { Rocky } \\
\text { Mountain } \\
\text { House }\end{array}$ & $\mathrm{AB}$ & 78 & $39,665.82$ & $31,291.6$ & $105,775.5$ & $83,444.26$ & 240.7046 & 813.6579 & 0.335412 & 0.4536 & 231.594 & $\begin{array}{l}\text { Lower Mannville } \\
\text { Group }\end{array}$ & 2.6 \\
\hline Saskatoon & Sask. & - & - & - & - & - & - & - & - & - & - & shallow basin & _- \\
\hline Slave Lake & $\mathrm{AB}$ & - & - & - & - & - & - & - & - & - & - & shallow basin & - \\
\hline Spruce Grove & $\mathrm{AB}$ & 40 & - & - & - & - & - & - & - & - & 79.86 & $\begin{array}{l}\text { Lower Mannville } \\
\text { Group }\end{array}$ & 1.3 \\
\hline St. Albert & $\mathrm{AB}$ & 40 & - & - & - & - & - & - & - & - & 79.86 & $\begin{array}{l}\text { Lower Mannville } \\
\text { Group }\end{array}$ & 1.2 \\
\hline St. Paul & $\mathrm{AB}$ & - & - & - & - & - & - & - & - & - & - & shallow basin & - \\
\hline Stettler & $\mathrm{AB}$ & 40.6 & - & - & - & - & - & - & - & - & 82.2558 & $\begin{array}{l}\text { Lower Mannville } \\
\text { Group }\end{array}$ & 1.4 \\
\hline Stony Plain & $\mathrm{AB}$ & 40 & - & - & - & - & - & - & - & - & 79.86 & $\begin{array}{l}\text { Lower Mannville } \\
\text { Group }\end{array}$ & 1.4 \\
\hline Swift Current & Sask. & 27 & - & - & - & - & - & - & - & - & 27.951 & shallow basin & 1 \\
\hline Taber & $\mathrm{AB}$ & - & - & - & - & - & - & - & - & - & - & shallow & - \\
\hline Three Hills & $\mathrm{AB}$ & - & - & - & - & - & - & - & - & - & 96.6306 & Lower Mannville & 1.7 \\
\hline Varman & Sask. & - & - & - & - & - & - & - & _- & _- & - & shallow basin & _- \\
\hline Vegreville & $\mathrm{AB}$ & - & - & - & - & - & - & - & - & - & - & shallow basin & - \\
\hline Vermilion & Man. & - & - & - & - & - & - & - & - & - & - & shallow & - \\
\hline Virden Man. & $\mathrm{AB}$ & - & - & - & - & - & - & - & - & - & - & shallow basin & - \\
\hline Wainwright & Sask. & - & - & - & - & - & - & - & - & - & - & shallow basin & - \\
\hline Westlock & $\mathrm{AB}$ & 32 & - & - & - & - & - & - & - & - & 47.916 & $\begin{array}{l}\text { Lower Mannville } \\
\text { Group }\end{array}$ & 1 \\
\hline Wetaskiwin & $\mathrm{AB}$ & 50 & - & - & - & - & - & - & - & - & 119.79 & $\begin{array}{l}\text { Lower Mannville } \\
\text { Group }\end{array}$ & 1.4 \\
\hline Weyburn & Sask. & 30.6 & - & - & - & - & - & - & - & - & 42.3258 & shallow basin & 0.9 \\
\hline White City & Sask. & - & - & - & - & - & - & - & - & - & - & shallow basin & - \\
\hline Whitecourt & $\mathrm{AB}$ & 65 & $11,018.28$ & 8692.11 & $29,382.09$ & $23,178.96$ & 66.86238 & 226.0161 & 0.179685 & 0.126 & 179.685 & $\begin{array}{l}\text { Lower Mannville } \\
\text { Group }\end{array}$ & 1.8 \\
\hline Yorkton & Sask. & _- & _- & - & _ & _- & _ & _- & _- & _- & - & shallow basin & _- \\
\hline
\end{tabular}




\section{References}

1. Agemar, T.; Weber, J.; Schulz, R. Deep Geothermal Energy Production in Germany. Energies 2014, 7, 4397-4416. [CrossRef]

2. Laplaige, P.; Lemale, J.; Decottegnie, S.; Desplan, A.; Goyeneche, O.; Delobelle, G. Geothermal Resources in France-Current Situation and Prospects. In Proceedings of the World Geothermal Congress, Antalya, Turkey, 1 April 2005.

3. Limberger, J.; Boxem, T.; Pluymaekers, M.; Bruhn, D.; Manzella, A.; Calcagno, P.; Beekman, F.; Cloetingh, S.; van Wees, J.-D. Geothermal energy in deep aquifers: A global assessment of the resource base for direct heat utilization. Renew. Sustain. Energy Rev. 2018. [CrossRef]

4. Lund, J.W. Direct Utilization of Geothermal Resources Worldwide. Energies 2010, 3. [CrossRef]

5. Tester, J.W.; Meissner, H.P.; Anderson, B.J.; Batchelor, A.S.; Blackwell, D.D.; DiPippo, R. The Future of Geothermal Energy: The Impact of Enhanced Geothermal Systems on the United States in the 21st Century; Cambridge Press: Cambridge, UK, 2006.

6. Grasby, S.E.; Allen, D.M.; Chen, Z.; Ferguson, G.; Jessop, A.; Kelman, M.; Majorowicz, J.; Moore, M.; Raymond, J.; Therrien, R. Geothermal Energy Resource Potential of Canada. In Geological Survey of Canada GSC Open File; GSC: Calgary, AB, Canada, 2011; 322p.

7. Majorowicz, J.; Moore, M. The feasibility and potential of geothermal heat in the deep Alberta foreland basin-Canada for $\mathrm{CO}_{2}$ savings. Renew. Energy 2014, 66, 541-549. [CrossRef]

8. Moeck, I. Catalog of geothermal play types based on geologic controls. Renew. Sustain. Energy Rev. 2014, 37, 867-882. [CrossRef]

9. Majorowicz, J.; Grasby, S.E. High Potential Regions for Enhanced Geothermal Systems in Canada. Nat. Resour. Res. 2010, 19, 177-188. [CrossRef]

10. Majorowicz, J.; Grasby, S.E. Heat flow, depth-temperature variations and stored thermal energy for enhanced geothermal systems in Canada. J. Geophys. Eng. 2010, 7, 232. [CrossRef]

11. Majorowicz, J.; Grasby, S.E. Deep geothermal energy in Canadian sedimentary basins vs. fossils based energy we try to replace -exergy [kJ/kg] compared. Renew. Energy 2019, 141, 259-277. [CrossRef]

12. Majorowicz, J.; Jessop, A. Regional heat flow patterns in the western Canadian sedimentary basin. Tectonophysics 1981, 74, 209-238. [CrossRef]

13. Jessop, A.M. Thermal Geophysics, 1st ed.; Elsevier: Amsterda, The Netherlands, 1990; pp. 1-306.

14. Majorowicz, J.A.; Garven, G.; Jessop, A.; Jessop, C. Present heat flow along a profile across the Western Canada Sedimentary Basin: The extent of hydrodynamic influence. In Geothermics in Basin Analysis; Springer: Berlin/Heidelberg, Germany, 1999; pp. 61-79.

15. Weides, S.; Majorowicz, J. Implications of spatial variability in heat flow for geothermal resource evaluation in large foreland basins: The case of the Western Canada Sedimentary Basin. Energies 2014, 7, 2573-2594. [CrossRef]

16. Majorowicz, J.; Weides, S. Large scale geothermal high in the westernmost North American covered craton-Can heat flow vs. basement heat production be a reliable tool in predicting deep EGS geothermal resource? In Proceedings of the 40th Workshop on Geothermal Reservoir Engineering, Stanford, CA, USA, 26-28 January 2015.

17. Majorowicz, J.; Grasby, S.E. Heat transition in Alberta, Canada: Geothermal energy prospects for major communities. Geothermics 2020, 88. [CrossRef]

18. Ammar, Y.; Joyce, S.; Norman, R.; Wang, Y.; Roskilly, A.P. Low grade thermal energy sources and uses from the process industry in the UK. Appl. Energy 2012, 89, 3-20. [CrossRef]

19. Kapil, A.; Bulatov, I.; Smith, R.; Kim, J. Process integration of low grade heat in process industry with district heating networks. Energy 2012, 44, 11-19. [CrossRef]

20. Tester, J.W.; Reber, T.; Beckers, K.; Lukawski, M.; Camp, E.; Andrea, G.; Aguirre, A.; Jordan, T.; Horowitz, F. Integrating Geothermal Energy Use into Re-building American Infrastructure. In Proceedings of the World Geothermal Congress 2015, Melbourne, Australia, 19-25 April 2015.

21. Jessop, A.; Vigrass, L. The Regina geothermal experiment-Thermal aspects. In Energy Developments: New Forms, Renewables, Conservation; Curtis, F.A., Ed.; Pergamon Press: Oxford, UK, 1984; pp. 315-320.

22. Nieuwenhuis, G.; Lengyel, T.; Majorowicz, J.; Grobe, M.; Rostron, B.; Unsworth, M.J.; Weides, S. Regional-scale geothermal exploration using heterogeneous industrial temperature data. A case study from the Western Canadian sedimentary Basin. In Proceedings of the World Geothermal Congress, Melbourne, Australia, 19-25 April 2015.

23. Majorowicz, J. Heat flow-heat production relationship not found: What drives heat flow variability of the Western Canadian foreland basin? Int. J. Earth Sci. 2016. [CrossRef]

24. Jones, F.W.; Majorowicz, J.A. Regional trends in radiogenic heat generation in the Precambrian basement of the Western Canadian Basin. Geophys. Res. Lett. 1987, 14, 268-271. [CrossRef]

25. Jessop, A.M. Thermal input from the basement of the Western Canada Sedimentary Basin. Bull. Can. Petroleum. Geol. 1992, 40, 198-206.

26. Majorowicz, J.; Nieuwenhuis, G.; Unsworth, M.J.; Phillips, J.; Verveda, R. High Temperatures Predicted in the Granitic Basement of Northwest Alberta-An assessment of the EGS Potential. In Proceedings of the Thirty-Ninth Workshop on Geothermal Reservoir Engineering; SGP-TR-202; Stanford University: Stanford, CA, USA, 2014; pp. 24-26. 
27. Majorowicz, J.; Grasby, S.E.; Skinner, W.R. Estimation of shallow geothermal energy resource in Canada: Heat gain and heat sink. Nat. Resour. Res. 2009, 18, 95-108. [CrossRef]

28. Pollack, H.N.; Chapman, D.S. Mantle heat flow. Earth Planet. Sci. Lett. 1977, 34, 174-184. [CrossRef]

29. Artemieva, I.M. The Lithosphere: An Interdisciplinary Approach; Cambridge University Press: New York, NY, USA, 2011; 794p, ISBN 9780521843966.

30. Artemieva, I.M.; Mooney, W.D. Thermal thickness, and evolution of Precambrian lithosphere: A global study. J. Geophys. Res. 2001, 106, 16387-16414. [CrossRef]

31. Eppelbaum, L.V.; Kutasov, I.M.; Pilchin, A.N. Applied Geothermics; Springer: Heidelberg, NY, USA, 2014.

32. Blackwell, D.D.; Richards, M. (Eds.) Heat Flow Map of North America, 1st ed.; AAPG: Tulsa, OK, USA, $2004 ;$ ISBN 0791815722.

33. Majorowicz, J.; Chan, J.; Crowell, J.; Gosnold, W.; Heaman, L.M.; Kuck, J.; Nieuwenhuis, G.; Schmitt, D.R.; Unsworth, M.J.; Walsh, N.; et al. The first deep heat flow determination in crystalline basement rocks beneath the Western Canadian Sedimentary Basin. Geophys. J. Int. 2014, 197, 731-747. [CrossRef]

34. MIT Seawater Thermophysical Properties Library. 2016. Available online: http://web.mit.edu/seawater/2017_MIT_Seawater_ Property_Tables_r2b.pdf (accessed on 12 October 2020).

35. Ferguson, G.; Ufondu, L. Geothermal energy potential of the Western Canada Sedimentary Basin: Clues from coproduced and injected water. Environ. Geosci. 2017, 24, 113-121. [CrossRef]

36. Grasby, S.E.; Chen, Z. Subglacial recharge into the Western Canada Sedimentary Basin: Impact of Pleistocene glaciation on basin hydrodynamics. Geol. Soc. Am. Bull. 2005, 117, 500-514. [CrossRef]

37. Lam, H.-L.; Jones, F. Geothermal energy potential in the Hinton-Edson area of west-central Alberta. Can. J. Earth Sci. 1985, 22, 369-383. [CrossRef]

38. Lam, H.-L.; Jones, F. An investigation of the potential for geothermal energy recovery in the Calgary area in southern Alberta, Canada, using petroleum exploration data. Geophysics 1986, 51, 1661-1670. [CrossRef]

39. Weides, S.N.; Moeck, I.S.; Schmitt, D.R.; Majorowicz, J.A. An integrative geothermal resource assessment study for the siliciclastic Granite Wash Unit, northwestern Alberta (Canada). Environ. Earth Sci. 2014, 72, 4141-4154. [CrossRef]

40. Weides, S.; Moeck, I.; Majorowicz, J.; Grobe, M. The Cambrian Basal Sandstone Unit in Central Alberta-An investigation of temperature distribution, petrography and hydraulic and geomechanical properties of a deep saline aquifer. Can. J. Earth Sci. 2014, 51, 783-796. [CrossRef]

41. Weides, S.; Moeck, I.; Majorowicz, J.; Palombi, D.; Grobe, M. Geothermal exploration of Paleozoic formations in Central Alberta. Can. J. Earth Sci. 2013, 50, 519-534. [CrossRef]

42. Jessop, A.M.; Vigrass, L.W. Geothermal measurements in a deep well at Regina, Saskatchewan. J. Volcan. Geothermal Res. 1989, 37, 151-166. [CrossRef]

43. Younger, P. Geothermal Energy: Delivering on the Global Potential. Energies 2015, 8, 1173711754. [CrossRef]

44. Lukawski, M.Z.; Anderson, B.J.; Augustine, C.; Capuano, L.E., Jr.; Beckers, K.F.; Livesay, B.; Tester, J.W. Cost analysis of oil, gas, and geothermal well drilling. J. Pet. Sci. Eng. 2014, 118, 1-14. [CrossRef]

45. Sass, I.; Weydt, L.M.; Götz, A.E.; Machel, H.G.; Heldmann, C.-D.J. Geothermal Reservoir Analogues on a Continental Scale: Western Canadian Sedimentary Basin versus Northern Alpine Molasse Basin. In Proceedings of the World Geothermal Congress, Reykjavik, Iceland, 26 April-2 May 2020.

46. Weydt, L.M.; Heldmann, C.-D.J.; Machel, H.G.; Sass, I. From oil field to geothermal reservoir: Assessment for geothermal utilization of two regionally extensive Devonian carbonate aquifers in Alberta, Canada. Solid Earth 2018, 9, 953-983. [CrossRef]

47. Ferguson, G.; Grasby, S.E. The geothermal potential of the basal clastics of Saskatchewan, Canada. Hydrogeol. J. 2014, 22, 143-150. [CrossRef]

48. DEEP: First Results Company Release. Available online: https:/ / deepcorp.ca/deep-successfully-completes-first-geothermaltest-well/ (accessed on 14 October 2019).

49. Banks, J.; Harris, N.B. Geothermal potential of Foreland Basins: A case study from the Western Canadian Sedimentary Basin. Geothermics 2018, 76, 74-92. [CrossRef]

50. Palmer-Wilson, K.; Banks, J.; Walsh, W.; Robertson, B. Sedimentary basin geothermal favourability mapping and power generation assessments. Renew. Energy 2018, 127, 1087-1100. [CrossRef] 